\title{
A CRISPR/Cas9 library to map the HIV-1 provirus genetic fitness
}

\author{
K. E. YODER
}

The Ohio State University College of Medicine, Cancer Biology and Genetics, 460 West $12^{\text {th }}$ Ave. Room 1006, Columbus, $\mathrm{OH} 43210$ USA

Received June 27, 2018; accepted September 27, 2018

\begin{abstract}
Summary. - The integrated proviral genome is the major barrier to a cure for HIV-1 infection. Genome editing technologies, such as CRISPR/Cas9, may disable or remove the HIV-1 provirus by introducing DNA double strand breaks at sequence specific sites in the viral genome. Host DNA repair by the error-prone nonhomologous end joining pathway generates mutagenic insertions or deletions at the break. CRISPR/Cas9 editing has been shown to reduce replication competent viral genomes in cell culture, but only a minority of possible genome editing targets have been assayed. Currently there is no map of double strand break genetic fitness for HIV-1 to inform the choice of editing targets. However, CRISPR/Cas9 genome editing makes it possible to target double strand breaks along the length of the provirus to generate a double strand break genetic fitness map. We identified all possible HIV-1 targets with different bacterial species of CRISPR/Cas9. This library of guide RNAs was evaluated for GC content and potential off-target sites in the human genome. Complexity of the library was reduced by eliminating duplicate guide RNA targets in the HIV-1 long terminal repeats and targets in the env gene. Although the HIV-1 genome is AT-rich, the S. pyogenes CRISPR/Cas9 with the protospacer adjacent motif NGG offers the most HIV-1 guide RNAs. This library of HIV-1 guide RNAs may be used to generate a double strand break genetic fragility map to be further applied to any genome editing technology designed for the HIV-1 provirus.
\end{abstract}

Keywords: HIV-1; genome editing; CRISPR; genetic fitness; guide RNAs

\section{Introduction}

As a retrovirus, human immunodeficiency virus 1 (HIV-1) integrates a copy of the viral genome into the host DNA of CD4+ T cells (Coffin et al., 1997). This integrated provirus may become transcriptionally silent, or latent (Finzi et al., 1999). HIV-1 infection can be controlled by treatment with a combination therapy of anti-retroviral drugs. In spite of persistently undetectable viral loads, the HIV-1 latent reservoir

E-mail: yoder.176@osu.edu; phone: +1-(614)-688-2106.

Abbreviations: CRISPR = clustered regularly interspersed short palindromic repeat; DSB = double strand break; FIV = feline immunodeficiency virus; gRNA = guide RNA; HIV-1 = human immunodeficiency virus 1 ; LTR = long terminal repeat; NHEJ = non-homologous end joining; $\mathrm{NM}=N$. meningitides; $\mathrm{PAM}=$ protospacer adjacent motif; $\mathrm{SA}=\mathrm{S}$. aureus $; \mathrm{SP}=$ S. pyogenes; $\mathrm{ST} 1=$ S. thermophilus persists for many years and quickly re-establishes infection when anti-retroviral therapy is stopped (Archin et al., 2012; Palmer et al., 2011). Latently infected cells prevent the cure of HIV-1 infection (Siliciano et al., 2003).

Genome editing technologies have been proposed as one strategy for a cure by deleting or disabling the latent HIV-1 provirus (Aubert et al., 2011; Ebina et al., 2013; Hu et al., 2014; Liao et al., 2015; Qu et al., 2013; Zhu et al., 2015). The proposed strategies have included homing endonucleases, zinc finger nucleases (ZFNs), transcription activator-like effector nucleases (TALENs), and clustered regularly interspersed short palindromic repeat (CRISPR/Cas9) (Aubert et al., 2011; Ebina et al., 2013; Manjunath et al., 2013; Qu et al., 2013). All of these genome editors target a double strand break (DSB) to a sequence specific location in target DNA. DSBs are most commonly repaired by error-prone non-homologous end joining (NHEJ), which introduces insertions or deletions (indels) at the repair junction (Lieber, 
2010). The generation of indels is often used to disrupt the reading frame of a gene of interest. Except for CRISPR/ Cas9, all of these genome editing technologies are sequence specific DNA binding proteins, requiring engineering of a protein to alter the target specificity. CRISPR/Cas9 sequence specificity is mediated by a guide RNA (gRNA) that has direct sequence homology to the DNA target. The relative ease of altering the target site of CRISPR/Cas9 editing and the ability to multiplex gRNAs make this technology highly attractive (Cong et al., 2013; Wang et al., 2013).

Clustered regularly interspersed short palindromic repeat is a rudimentary immune system in bacteria that disables foreign genetic elements (Makarova et al., 2011). The S. pyogenes (SP) Type II CRISPR has become a common research tool (Shalem et al., 2014; Wang et al., 2013). In this CRISPR system a single protein, Cas9, binds a constant sequence scaffold RNA linked to a variable 20 base gRNA that is homologous to a DNA target site. CRISPR/Cas9 genomic targeting requires a protospacer adjacent motif (PAM) located in the target DNA at the 3' end of the gRNA target site sequence (Sternberg et al., 2014). The PAM is recognized by the Cas9 protein during the target search but is not included in the gRNA sequence. Cas9 contains two endonuclease domains that introduce a blunt end DSB into the genomic DNA 3 bp 5 ' to the PAM (Gasiunas et al., 2012; Nishimasu et al., 2014). The PAM varies between bacterial species; for example, the SP PAM is NGG, the N. meningitides (NM) PAM is NNNGMTT, the $S$. thermophilus (ST1) Cas9 PAM is NNAGAAW, and the $S$. aureus (SA) Cas9 PAM is NNGRRT (Cong et al., 2013; Esvelt et al., 2013; Garneau et al., 2010; Ran et al., 2015). The HIV-1 genome is AT-rich with $<43 \%$ GC content, suggesting a Cas9 with a more AT rich PAM might have more targets in the HIV-1 genome.

CRISPR/Cas9 genome editing strategies are intriguing approaches to combat HIV-1 infection. CRISPR/Cas9 targeted to the host HIV-1 co-receptor gene CCR5 has shown clinical promise, but is still inefficient for HIV-1 eradication (Tebas et al., 2014). In the case of targeting the HIV-1 provirus, NHEJ associated indels could alter the reading frame of the viral genes (Wang et al., 2016a,b,c; Yin et al., 2016). Indels have also been proposed to alter critical RNA secondary structures by altering their coding DNA regions (Ebina et al., 2013; Lebbink et al., 2017; Yin et al., 2016; Yoder and Bundschuh, 2016). In addition, targeting of the long terminal repeat (LTR) sequences that flank the HIV-1 proviral genome has been suggested as a mechanism to delete the viral genome (Ebina et al., 2013; Hu et al., 2014; Kaminski et al., 2016a; Kaminski et al., 2016b). This provirus deletion strategy is likely to be inefficient compared to generation of indels at the two targets independently (Canver et al., 2014). CRISPR/ Cas9 has been shown to disable both latent and replicating HIV-1 (Ebina et al., 2013; Hu et al., 2014; Liao et al., 2015; Zhu et al., 2015). Importantly, genome editing by CRISPR/
Cas9 does not necessarily require reactivation of latent HIV-1 proviruses (Zhu et al., 2015). Studies of CRISPR/Cas9 editing of the HIV-1 proviral genome have used sets of 2 to 26 targeting gRNAs and SP Cas9; one study utilized SA Cas9 with 2 gRNAs (Ebina et al., 2013; Hu et al., 2014; Kaminski et al., 2016a; Kaminski et al., 2016b; Lebbink et al., 2017; Liao et al., 2015; Ueda et al., 2016; Wang et al., 2016a,b,c; Yin et al., 2016; Yin et al., 2017; Yoder and Bundschuh, 2016; Zhu et al., 2015). As with any monotherapy, HIV-1 strains resistant to a single CRISPR gRNA arose (Wang et al., 2016b,c; Yoder and Bundschuh, 2016). Some of the observed resistance mutations developed during the mutagenic process of reverse transcription (Wang et al., 2016b; Yoder and Bundschuh, 2016). In addition, CRISPR gRNA resistant strains were generated by the error-prone NHEJ DNA repair of the Cas9 induced DSB (Wang et al., 2016b,c; Yoder and Bundschuh, 2016). Indels at the repair junction conferred resistance to further cleavage and thus resistant strains. In non-coding regions the indels were typically a single bp, while coding regions included 3 bp indels (Yoder and Bundschuh, 2016).

The efficiency of any genome editing strategy of the HIV-1 provirus is inherently dependent on the genetic fragility of the target site. Genetic fitness may vary from fragile to robust throughout the viral genome. Fragile regions of the genome are not tolerant of mutations and reduce viral replication; genetically robust sites tolerate mutations and have little or no effects on viral replication. The measure of genetic fitness is not limited to the type of mutation and may include both substitution mutations and indel mutations (de Visser et al., 2003). However, the current map of the HIV-1 genome fitness landscape is derived from sequencing studies of patient isolates (Zanini et al., 2017). This map of genetic fitness is largely limited to substitution mutations. Previous studies mapped the genetic robustness of HIV-1 capsid and integrase genes by single amino acid substitutions (Rihn et al., 2015; Rihn et al., 2013). Genetic robustness was measured by the replication capacity of mutant viruses. Although both capsid and integrase proteins form multimer complexes, capsid was found to be more genetically fragile and intolerant of mutations. In capsid 70\% of amino acid substitutions inactivated the virus, compared to only $35 \%$ of integrase mutations (Rihn et al., 2013, 2015). Thus, integrase is more genetically robust than capsid. The selective pressures of patient infection or engineered substitution mutations do not necessarily reflect the pressures of DSBs and/or indels. Importantly, the map of HIV-1 genetic fitness based on substitution mutations will likely be different than the map based on DSB induced indel mutations.

The concept of DSB genetic fragility has not been applied to the choice of CRISPR gRNAs targeting the HIV-1 genome. The choice of gRNAs has largely been based on predicted Cas9 cleavage efficiency or sequence conservation among HIV-1 subtype B strains (Hu et al., 2014; Kaminski et al., 2016b; Liao et al., 2015; Wang et al., 2016a,b; Yin et al., 2016; 
Yoder and Bundschuh, 2016; Zhu et al., 2015). One previous study compared the sequences of gRNA resistant strains to the HIV-1 subtype B patient isolates in the Los Alamos National Laboratory HIV Databases (Yoder and Bundschuh, 2016). Many of the resulting resistant strains displayed single base indels and are not present in the HIV-1 databases of patient isolates (Yoder and Bundschuh, 2016). In contrast, the mutations in HIV-1 strains that developed resistance to siRNA inhibition are present in the HIV-1 patient isolate databases (ter Brake et al., 2008; von Eije et al., 2008). These observations suggest that while a substitution mutation genome fragility map of HIV-1 may successfully be applied to the choice of inhibitory siRNAs, it will have limited utility with CRISPR/Cas9 genome editing. Instead a new DSB associated genetic fragility map of HIV-1 is required as a foundation for genome editing target predictions.

A DSB genetic fitness map of the HIV-1 genome may be derived from a CRISPR/Cas9 gRNA library targeting the provirus. In contrast to scanning alanine mutagenesis which is necessarily limited to protein coding regions, a CRISPR gRNA library has the ability to assay the fragility of non-coding viral sequences. The gRNAs that do not affect HIV-1 replication will be considered to target genetically robust sites of the viral genome. The gRNAs that delay HIV-1 replication will indicate that the targets are genetically fragile sites. We have computationally derived a comprehensive set of CRISPR gRNAs that may be assayed in a 96 well format to model DSB genetic fitness throughout the HIV-1 genome. This gRNA library assay may also be applied to off-target editing. Thorough quantitation and stratification of targets throughout the HIV-1 genome for genetic fragility and potential off-target editing will inform future strategies based on any genome editing technology.

\section{Materials and Methods}

The sequences of HIV-1 strains LAI (GenBank K02013.1), NL4-3 (GenBank AF324493.2), BK132 (GenBank AY173951), 671 (GenBank AY423387), and 1058 (GenBank AY331295) were input as fasta sequences to the web-based tool chopchop.cbu.uib.no (Leitner et al., 2005; Montague et al., 2014; Wain-Hobson et al., 1985). HIV-1 strain LAI was analyzed for SP, NM, and ST1 editing with the CRISPR/Cas9 option and for Cpf1 editing with the CRISPR/ Cpf1 option. Strains NL4-3, BK132, 671, and 1058 were evaluated for SP targets. The targets were evaluated in the background of the H. sapiens (hg38/GRCh38) genome. The PAM sequence options were NGG for SP, NNNGMTT for NM, NNAGAAW for ST1, and NNGRRT for SA. The method for identifying off-target sites in the human genome was set to no more than 0 mismatches in the 15 bases adjacent to the PAM, the protospacer seed region (Cong et al., 2013). The gRNAs were mapped to the respective HIV-1 proviral features based on the GenBank annotations.

\section{Results}

Generating a library of $g R N A$ s targeting the HIV-1 genome

LAI was one of the first HIV-1 strains cultured from a patient and subsequently sequenced (Wain-Hobson et al., 1991). The proviral sequence was analyzed for gRNAs with the web-based tool chopchop.cbu.uib.no (Montague et al., 2014; Wain-Hobson et al., 1985). This website allows the analysis of a target sequence for genome editing by Cas9 proteins from multiple bacterial species with different PAM sequence requirements. SP CRISPR/Cas9 with an NGG PAM signal was the first described for use in mammalian cells (Cho et al., 2013; Cong et al., 2013). However, the HIV-1 genome is AT-rich with $\sim 40 \%$ GC content. We considered that Cas9 proteins with a more AT rich PAM sequence might generate better coverage of the HIV-1 genome. The ST1 Cas9 PAM is NNAGAAW and the NM Cas9 PAM is NNNGMTT (Esvelt et al., 2013; Garneau et al., 2010). The SA Cas9 with PAM NNGRRT can be AT rich (Ran et al., 2015). These Cas 9 proteins function in the same way as the SP Cas9, digesting the target DNA 3 bp 5 ' of the PAM sequence. The probability of off-target editing was reduced by excluding gRNAs that perfectly matched a genomic sequence in the $3^{\prime} 15 \mathrm{bp}$ of the gRNA (Cong et al., 2013; Montague et al., 2014). The total gRNAs identified were 1046 targets with SP Cas9, 160 targets with NM Cas9, 83 targets with ST1 Cas9, and 283 targets with SA Cas9 (Table 1). Although the HIV-1 genome is AT-rich, the shorter SP PAM sequence is more abundant.

We also considered an alternative genome editing protein Cpf1 from Francisella tularensis subsp. novicida (Zetsche et al., 2015). The Cpf1 PAM TTTN is on the 5 ' side of the gRNA target sequence. The enzyme generates a staggered cleavage 18-23 base pairs on the 3' side of the PAM. There are 554 Cpf1 gRNA target sites in the HIV-1 LAI genome (Table 1). Surprisingly, the total number of SP Cas9 target gRNAs was greater than any genome editor with an AT rich PAM.

Table 1. CRISPR/Cas9 gRNAs from 5 different bacterial species targeting the HIV-1 LAI genome

\begin{tabular}{lccccc}
\hline & SP & NM & ST1 & SA & Cpf1 \\
\hline Total gRNAs & 1046 & 160 & 83 & 283 & 554 \\
GC $<40 \%$ & 323 & 60 & 34 & 96 & 255 \\
GC $>70 \%$ & 26 & 1 & 0 & 6 & 7 \\
Mismatches & 40 & 0 & 0 & 1 & 7 \\
Duplicates in LTR & 71 & 12 & 0 & 16 & 12 \\
env gene & 112 & 11 & 13 & 34 & 47 \\
Pared gRNAs & 474 & 76 & 36 & 130 & 226 \\
Accessory genes & 88 & 10 & 11 & 26 & 36 \\
Pared gRNAs & & & & & \\
(HeLa assay) & 386 & 66 & 25 & 104 & 190 \\
\hline
\end{tabular}




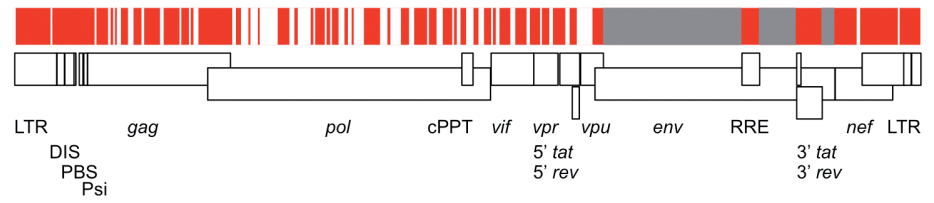

Fig. 1

Scale map of SP gRNAs targeting the HIV-1 genome

A cartoon of the HIV-1 LAI proviral genome is shown to scale in white boxes. Below the genome are the genomic elements, such as the LTRs, dimerization initiation site (DIS), primer binding site (PBS), genomic RNA packaging signal (Psi), central polypurine tract (cPPT), and Rev response element (RRE), as well as the viral genes in italics. S. pyogenes (SP gRNA) binding sites are shown in red. Gaps in gRNA coverage $\geq 20$ bp are white spaces. Regions of the env gene that are excluded from the gRNA library are grey.

The gRNA targets were further analysed, and several criteria were used to exclude gRNAs (Table 1). The lists were pared by removing gRNAs with G/C content $<40 \%$ or $>70 \%$ since they are likely to be inefficient. All gRNAs with $\geq 1$ mismatch with a genomic off-target site were excluded. The gRNA list includes targets in both LTRs; duplicate gRNAs were discarded. The HIV-1 env gene has been shown to be genetically divergent in patients and tolerant of mutations; gRNAs targeting the env gene were excluded (Hahn et al., 1986). However, regions of env that also encode tat, rev, or the Rev response element (RRE) RNA stem-loop structure were included. According to these criteria, the final numbers of gRNAs were 474 SP Cas 9 gRNAs, 76 NM Cas 9 gRNAs, 36 ST1 Cas9 gRNAs, 130 SA Cas9 gRNAs, and 226 Cpf1 gRNAs (Table 1, Supplementary Table 1).

While the SP Cas9 library of gRNAs appears to offer the best coverage of the HIV-1 genome, some of the gRNA sequences overlap. In order to better visualize coverage of the LAI genome, SP gRNAs were mapped to scale (Fig. 1). There were 89 gaps in the SP gRNA coverage of the HIV-1 LAI genome. Most of the gaps were $<20 \mathrm{bp}$. The LTR sequences that flank the HIV-1 genome had only one 21 bp gap. The pol gene displayed the most gaps in SP gRNA coverage with only $48 \%$ of the gene targeted by 145 gRNAs. Since Cpf1 targets were more abundant than other Cas 9 targets, we considered whether the Cpfl gRNA coverage of pol might be more complete than SP gRNAs. There are 103 Cpf1 gRNAs

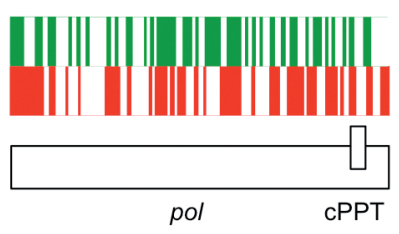

Fig. 2

Scale map of SP and Cpf1 gRNAs targeting the HIV-1 LAI pol gene A cartoon of the LAI pol gene is shown as a box with the region also encoding the cPPT genetic element indicated by a smaller box. The SP gRNA recognition sites are shown to scale in red. Cpf1 gRNA binding sites are in green. Gaps between gRNAs $\geq 20$ bp are white space. targeting $47 \%$ of the pol gene. Mapping the Cpf1 gRNAs to the LAI pol gene reveals that many of the gaps in coverage are common to SP Cas9 and Cpf1 (Fig. 2). Combined libraries of Cpf1 and SP gRNAs would only cover $67.5 \%$ of the HIV-1 LAI pol gene. The SP gRNA library affords the most comprehensive editing of the LAI genome providing for the determination of a DSB associated genetic fitness map of the HIV-1 genome.

\section{Application of the LAI gRNA library to other HIV-1 strains}

As a screening tool, this HIV-1 gRNA library could conceivably be used to analyze other HIV-1 strains. We compared the gRNA library to the commonly used HIV-1 laboratory strain NL4-3 (Table 2). In addition, sequences of patient-derived reference strains BK132 from Thailand, 671 from Netherlands, and 1058 from USA were analyzed for complementarity with the LAI gRNA library (Table 2). Laboratory strain NL4-3 has $98 \%$ sequence identity to LAI, the highest of the strains evaluated. However, only 288 of 474 LAI gRNAs also targeted NL4-3. The patient derived strains display less sequence identity to LAI. Only 194, 141, or 113 LAI gRNAs also target BK132, 671, or 1058, respectively. As the LAI sequence identity of each strain decreased, the number of complementary LAI gRNAs also decreased. In contrast, an inverse correlation was observed between LAI sequence identity and the number of gRNAs unique to each strain. Further analysis of the patient strains and LAI revealed that only 53 gRNA target sequences are common

Table 2. Comparison of LAI to HIV-1 strains NL4-3, BK132, 671 , and 1058

\begin{tabular}{lccc}
\hline Strain & LAI identity & LAI gRNAs & Unique gRNAs \\
\hline NL4-3 & $98 \%$ & 288 & 193 \\
BK132 & $94 \%$ & 194 & 256 \\
671 & $92 \%$ & 141 & 337 \\
1058 & $92 \%$ & 113 & 339 \\
\hline
\end{tabular}




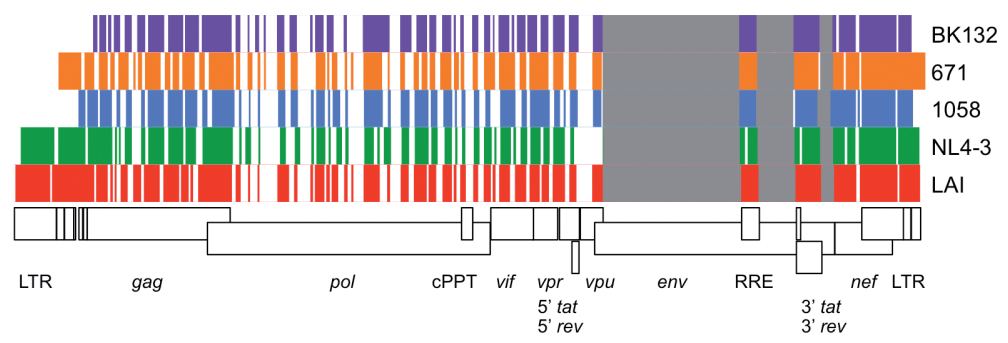

Fig. 3

Comparison of SP gRNAs targeting multiple HIV-1 strains

A scale map of the HIV-1 genome is shown in white boxes. SP Cas9 gRNA libraries targeting five HIV-1 strains are shown. Colored bars indicate gRNA binding sites. White spaces indicate gaps in coverage $\geq 20 \mathrm{bp}$. Regions of the env gene that are excluded from the gRNA library are grey. Libraries of gRNAs targeting HIV-1 strains LAI (red), NL4-3 (green), and patient strains BK132 (purple), 671 (orange), and 1058 (blue) are shown.

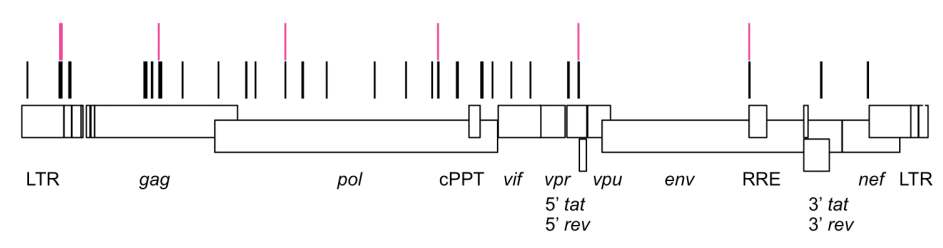

Fig. 4

SP gRNAs at conserved HIV-1 sequences

A scale map of the HIV-1 genome is shown in white boxes. SP Cas9 gRNAs that target five HIV-1 strains, shown in Fig. 3, are indicated by black lines. Previously characterized SP gRNAs are shown in pink.

to these 4 strains. These results suggest that the LAI gRNA library would not be an effective screening tool for other viral strains or patient quasi-species.

The patient strains and NL4-3 were evaluated for SP gRNA targets with the same methodology as LAI (Table 3). In all cases, the total number of possible gRNAs for each strain was less than LAI, ranging from 925 to 1029 total gRNAs identified. This is partially due to the incomplete LTR sequences for the patient strains. The pared lists of gRNAs ranging from 450 to $481 \mathrm{gRNAs}$ were nearly equal or slightly greater than 474 LAI gRNAs. However, the total number of gRNAs is not necessarily indicative of correlation in the viral genomes. While the LAI gRNA library is likely not suitable for screening other viral strains, none of the gRNA libraries

Table 3. SP gRNAs in NL4-3, BK132, 671, and 1058

\begin{tabular}{lcccc}
\hline & NL4-3 & BK132 & HIV 671 & HIV 1058 \\
\hline Total gRNAs & 1029 & 939 & 996 & 925 \\
GC $<40 \%$ & 331 & 313 & 365 & 321 \\
GC $>70 \%$ & 24 & 23 & 15 & 16 \\
Mismatches & 47 & 33 & 45 & 38 \\
Duplicates in LTR & 36 & 0 & 4 & 0 \\
env gene & 110 & 120 & 89 & 100 \\
Pared gRNAs & 481 & 450 & 478 & 450 \\
\hline
\end{tabular}

from these other strains displayed any superiority to the LAI gRNA library.

The gRNA libraries were mapped to scale for each genome (Fig. 3). Comparison of the gRNA maps for all of the strains shows impressive correlation of coverage. All of the strains show significant gaps in coverage of the pol and $v p u$ genes. In contrast, there are few gaps in coverage at several regions. For example, the LTRs offer many gRNA targets. We have previously targeted the LTRs with five different gRNAs and found that resistance mutations readily occur at these noncoding regions (Yoder and Bundschuh, 2016). The sequence surrounding the TATA box and TAR RNA stem loop coding regions are highly conserved suggesting they are genetically fragile and intolerant of mutations. However, when targeted by CRISPR/Cas9 these sequences proved to be genetically robust and tolerant of indels. More intriguing targets of the HIV-1 genome are those that have overlapping reading frames or RNA elements. For example, the regions of env that also encode the RRE RNA stem loop or that also encode the 3 ' exons of tat and rev. Although the specific gRNA sequences are not conserved between HIV-1 strains, comparison of the gRNA maps suggests the DSB genetic fragility map derived from LAI may be applied to other HIV-1 strains.

While the gRNA coverage maps appear similar, there are only 41 gRNA sequences in common between all strains 
analyzed. When mapped to the HIV-1 genome, it is readily apparent that these gRNAs occur throughout the provirus; they are not clustered in one gene or region (Fig. 4). Several of these gRNAs of highly conserved sequences have been previously characterized (Wang et al., 2016b,c; Yin et al., 2016; Yoder and Bundschuh, 2016; Zhu et al., 2015). Although the sequence conservation suggests genetic robustness, these sites proved in all cases to not be the strongest inhibitors of HIV-1 replication. Thus, the DSB genetic fragility appears to be demonstrably different from the currently available genetic fitness maps based on sequence conservation and substitution mutations.

\section{Discussion}

CRISPR/Cas9 genome editing is a relatively new technology that creates DSBs in a genome of interest (Cong et al., 2013; Esvelt et al., 2013; Hsu et al., 2013; Kim and Kim, 2014). The advantage of CRISPR/Cas9 genome editing compared to zinc finger nucleases or TALENs is the inherent ease of redirecting the DSB targeting via a gRNA rather than protein engineering. Generating a library of gRNAs is facile and offers the possibility of comparing multiple target sites in a high throughput screen. Our computational modeling identified 474 SP CRISPR/Cas9 gRNA targets throughout the HIV-1 LAI reference genome. This CRISPR/Cas9 gRNA library can induce DSBs throughout the length of the HIV-1 genome exerting novel pressures to select for resistant viral strains. The HIV-1 proviral genome does not experience this kind of DSB assault during infection of a patient. Thus, the HIV-1 genetic fitness map generated by patient samples may not faithfully recapitulate the fitness associated with induced DSBs. Direct comparison of these gRNAs can generate a new HIV-1 DSB genetic fitness map that will guide targeting of future genome editing strategies.

The HIV-1 LAI gRNA library proposed here may be implemented in a 96 well format. To evaluate the DSB genetic fitness of the HIV-1 genome, the CRISPR/Cas9 genome editing should occur in cells with replicating virus. Genetic fitness under pressure of genome editing DSBs is measured by HIV-1 replication dynamics in the presence of the gRNA compared to its absence. The gRNA library oligonucleotide synthesis and subcloning may be commercially subcontracted and distributed in a 96 well plate format. The cell type for screening the gRNA library should permit viral replication while also being amenable to transfection. Human CD4+ T cell lines readily allow HIV-1 replication, but these suspension cell lines are typically difficult to transfect. Hela cells have been engineered to express the human CD4 gene, the receptor for HIV-1 entry (Chesebro et al., 1990; Chesebro and Wehrly, 1988; Maddon et al., 1986). Adherent Hela-CD4 cells allow HIV-1 replication and may be efficiently transfected with the CRISPR/Cas9 gRNA library with lipid-based reagents. One important consideration of commercially available CRISPR/Cas9 delivery systems is that the lentiviral backbone may also be targeted by the gRNA library. The CRISPR/Cas9 system should be in a vector that does not include HIV-1 lentiviral sequences, which could be a similar feline immunodeficiency virus (FIV) based lentiviral vector (Poeschla et al., 1998). This alternative lentiviral vector has similar delivery properties as an HIV-1 based vector but will not be targeted by the HIV-1 gRNAs. FIV vectors may also encode a puromycin resistance gene allowing selection of successfully transduced cells. Transduced Hela-CD4 cells may be selected with puromycin generating stable Hela-CD4-Cas9 cells.

One limitation of performing this screen in a Hela derived cell line is that the accessory genes of HIV-1 - vif, vpr, vpu or nef - will not be functional in these cells. Although the gRNA library will target these genes, there will be no true selective pressure at these sites. However, Hela cell lines are highly amenable to the development of library screening tools due to their ease of transduction and transfection (Brass et al., 2008; Espeseth et al., 2011; Zhou et al., 2008). Removal of gRNAs that will be non-functional in Hela cells reduces the total number of SP gRNAs targeting LAI to 389. Once this CRISPR gRNA library screen has been developed in Hela cells, it may be adapted to different cell types, such as human CD4+ T cells, that will provide a selective test of the accessory genes.

The quantitation of HIV-1 replication in Hela-CD4-Cas9 cells may be performed in a 96 well plate format. Virus replication dynamics over time may be measured in two ways. First, the relative amount of infectious virus may be determined by a cellular assay, such as TZM-bl indicator cells, a Hela derived cell line that expresses beta-galactosidase and luciferase when infected with HIV-1 (Derdeyn et al., 2000; Platt et al., 1998, 2009; Platt et al., 1998; Takeuchi et al., 2008; Wei et al., 2002). Infectious HIV-1 virions released by the Hela-CD4-Cas9 cells may be measured by transferring media to TZM-bl indicator cells in a 96 well plate. Quantitation of luciferase or beta-galactosidase activity is a relative measure of infectious virus. Second, HIV-1 virus particles in the media may be directly measured by an ELISA for the capsid protein $\mathrm{p} 24$. Together these two assays allow for the quantitation of infectious activity of virions and of virus particles, which may be different. For example, successful genome editing of the HIV-1 integrase gene may allow secretion of virus particles to the media, but the defective integrase protein will render them noninfectious, and will not produce a signal in TZM-bl cells. Alternatively, genome editing of the HIV-1 promoter may prevent production of viral proteins and thus viral particles, ultimately resulting in reduced measurements in both the ELISA and TZM-bl assays. To stratify the gRNA library, primary weight is given 
to data from cellular infections. For gRNAs at genetically robust sites, viral replication will be equal to untreated cells. In contrast, cultures that continue to suppress viral replication for extended times will have gRNAs targeting genetically fragile sites that are intolerant of mutations. In these cases, ELISA quantitation of virus particles could stratify these gRNA targets. In some cases, there will be no detectable virus particles. However, it is possible that virus particles are produced, but are not infectious, such as an integrase or reverse transcriptase mutant virus. Together these values will rank the DSB genetic fitness of the HIV-1 genome.

One significant complication of all genome editing technologies is their propensity to target additional spurious sites (Cong et al., 2013). The human genome encodes endogenous retrovirus elements that are potential off-target editing sites for HIV-1 gRNAs. Although the HIV-1 gRNA library has been specifically designed to reduce off-target editing, the library should be empirically tested. Several previous reports of HIV-1 genome editing have relied on the Surveyor nuclease assay to test off-target editing. In this assay, potential off-target sites are predicted in silico (Hu et al., 2014; Liao et al., 2015; Yin et al., 2017; Yoder and Bundschuh, 2016). This assay is inherently biased by the prediction of off-target sites in silico. Alternative methods to assay for off-target editing depend on deep sequencing or whole genome sequencing. These methods are not feasible for a library of gRNAs. Instead, an unbiased assay adaptable to a multi-well format is preferred. An unbiased high throughput assay for offtarget genome editing takes advantage of the predilection for DSB repair to insert exogenous fragments of DNA nonspecifically (Gabriel et al., 2011; Wang et al., 2015b). First, cells are transiently transfected with the Cas9 gene and the gRNA. After 6 and 24 hours, the cells are transduced with an integrase-defective lentiviral vector encoding a puromycin selection gene. Importantly, this lentiviral vector should not be derived from HIV-1; instead an FIV based vector is more appropriate to avoid editing of the puromycin delivery genome. If off-target editing occurs, then the DSB may be repaired with the lentiviral genome encoding puromycin. Two days following transduction, the cells are put into drug selection. Following selection, the remaining viable cells may be measured by crystal violet staining. Only HIV-1 gRNAs that also edit the human genome at off-target sites will produce puromycin resistant cells. This assay is not biased by in silico prediction of off-target sites and is easily performed in multiwell plates. HIV-1 gRNAs that produce puromycin resistant cells will indicate regions of the HIV-1 genome that are significantly associated with off-target editing in the human genome. While these data do not inform the map of genetic fitness, it does indicate regions of the HIV-1 genome that should not be explored for future genome editing.

HIV-1 readily develops resistance to any mono-therapy, including single CRISPR/Cas9 gRNAs (Wang et al., 2016b,c;
Yoder and Bundschuh, 2016). Typically, mutations that confer resistance to a single anti-retroviral drug are generated during the error-prone reverse transcription of the viral single strand RNA genome to a double strand cDNA. Resistance to CRISPR/Cas9 genome editing is due to mutations induced by both reverse transcription and the error-prone DSB repair NHEJ pathway (Wang et al., 2016b,c; Yoder and Bundschuh, 2016). Thus, any single gRNA is likely to be inefficient in patient therapy. Two gRNAs capable of long-term suppression of replication of an HIV-1 lab strain in a cell line have been reported (Wang et al., 2016a,b). These studies identified highly fragile DSB sites in the gag and pol genes. These gRNA targets do not display the highest sequence conservation among HIV-1 subtype B isolates, underscoring the concept that sequence conservation and DSB associated fragility are independent. While resistance to these gRNAs did not develop under constrained laboratory conditions, a single gRNA is not likely to be effective for patient treatment. Instead, multiple CRISPR/Cas9 gRNAs that target multiple fragile sites could be multiplexed to effectively disable the HIV-1 quasispecies present in a patient. The sequence divergence within patients will likely necessitate a multiplexed genome editing system (Panfil et al., 2018). Currently the CRISPR/Cas9 system is the only genome editor capable of efficient multiplexing.

The major obstacle to an HIV-1 cure is the latent reservoir of long-lived cells with an integrated provirus (Palmer et al., 2011; Siliciano et al., 2003). The latent reservoir is not well understood, but these cells are known to be rare. Studies in cell lines suggest that CRISPR/Cas9 may effectively edit the latent provirus (Ebina et al., 2013; Hu et al., 2014; Zhu et al., 2015). Unfortunately, the use of CRISPR/Cas9 genome editing is limited by effective cellular targeting technologies. It is not clear how an effective CRISPR/Cas9 editor could be delivered to latently infected cells in a patient. However, novel CRISPR/Cas9 delivery schemes continue to be developed (Chiou et al., 2015; Cottle et al., 2015; Han et al., 2015; Wang et al., 2015a). Whether CRISPR/Cas9 genome editing of the HIV-1 provirus will prove to be clinically useful is unknown. Any alternative genome editing technology targeting the integrated provirus will benefit from a DSB genetic fragility map of the HIV-1 genome.

Supplementary information is available in the online version of the paper.

\section{References}

Archin NM, Vaidya NK, Kuruc JD, Liberty AL, Wiegand A, Kearney MF, Cohen MS, Coffin JM, Bosch RJ, Gay CL, Eron JJ, Margolis DM, Perelson AS (2012): Immediate antiviral therapy appears to restrict resting CD4+ cell HIV-1 in- 
fection without accelerating the decay of latent infection. Proc. Natl. Acad. Sci. USA 109, 9523-9528. https://doi. org/10.1073/pnas.1120248109

Aubert M, Ryu BY, Banks L, Rawlings DJ, Scharenberg AM, Jerome KR (2011): Successful targeting and disruption of an integrated reporter lentivirus using the engineered homing endonuclease Y2 I-AniI. PLoS One 6, e16825. https://doi. org/10.1371/journal.pone.0016825

Brass AL, Dykxhoorn DM, Benita Y, Yan N, Engelman A, Xavier RJ, Lieberman J, Elledge SJ (2008): Identification of host proteins required for HIV infection through a functional genomic screen. Science 319, 921-926. https://doi. org/10.1126/science.1152725

Canver MC, Bauer DE, Dass A, Yien YY, Chung J, Masuda T, Maeda T, Paw BH, Orkin SH (2014): Characterization of genomic deletion efficiency mediated by clustered regularly interspaced palindromic repeats (CRISPR)/Cas9 nuclease system in mammalian cells. J. Biol. Chem. 289, 21312-21324. https://doi.org/10.1074/jbc.M114.564625

Chesebro B, Buller R, Portis J, Wehrly K (1990): Failure of human immunodeficiency virus entry and infection in CD4positive human brain and skin cells. J. Virol. 64, 215-221. https://doi.org/10.1093/infdis/163.1.64

Chesebro B, Wehrly K (1988): Development of a sensitive quantitative focal assay for human immunodeficiency virus infectivity. J. Virol. 62, 3779-3788.

Chiou SH, Winters IP, Wang J, Naranjo S, Dudgeon C, Tamburini FB, Brady JJ, Yang D, Gruner BM, Chuang CH, Caswell DR, Zeng H, Chu P, Kim GE, Carpizo DR, Kim SK, Winslow MM (2015): Pancreatic cancer modeling using retrograde viral vector delivery and in vivo CRISPR/ Cas9-mediated somatic genome editing. Genes Dev. 29, 1576-1585. https://doi.org/10.1101/gad.264861.115

Cho SW, Kim S, Kim JM, Kim JS (2013): Targeted genome engineering in human cells with the Cas9 RNA-guided endonuclease. Nat. Biotechnol. 31, 230-232. https://doi. org/10.1038/nbt.2507

Coffin JM, Hughes SH, Varmus HE (1997): Retroviruses. Cold Spring Harbor Laboratory Press, Cold Spring Harbor.

Cong L, Ran FA, Cox D, Lin S, Barretto R, Habib N, Hsu PD, Wu X, Jiang W, Marraffini LA, Zhang F (2013): Multiplex genome engineering using CRISPR/Cas systems. Science 339, 819-823. https://doi.org/10.1126/science.1231143

Cottle RN, Lee CM, Archer D, Bao G (2015): Controlled delivery of beta-globin-targeting TALENs and CRISPR/Cas9 into mammalian cells for genome editing using microinjection. Sci. Rep. 5, 16031. https://doi.org/10.1038/srep16031

de Visser JA, Hermisson J, Wagner GP, Ancel Meyers L, BagheriChaichian H, Blanchard JL, Chao L, Cheverud JM, Elena SF, Fontana W, Gibson G, Hansen TF, Krakauer D, Lewontin RC, Ofria C, Rice SH, von Dassow G, Wagner A, Whitlock MC (2003): Perspective: Evolution and detection of genetic robustness. Evolution 57, 1959-1972. https://doi.org/10.1111/j.0014-3820.2003.tb00377.x

Derdeyn CA, Decker JM, Sfakianos JN, Wu X, O'Brien WA, Ratner L, Kappes JC, Shaw GM, Hunter E (2000): Sensitivity of human immunodeficiency virus type 1 to the fusion inhibitor T-20 is modulated by coreceptor specificity defined by the V3 loop of gp120. J. Virol. 74, 8358-8367. https://doi.org/10.1128/JVI.74.18.8358-8367.2000

Ebina H, Misawa N, Kanemura Y, Koyanagi Y (2013): Harnessing the CRISPR/Cas9 system to disrupt latent HIV-1 provirus. Sci. Rep. 3, 2510. https://doi.org/10.1038/srep02510

Espeseth AS, Fishel R, Hazuda D, Huang Q, Xu M, Yoder K, and Zhou H (2011): siRNA screening of a targeted library of DNA repair factors in HIV infection reveals a role for base excision repair in HIV integration. PLoS One 6, e17612. https://doi.org/10.1371/journal.pone.0017612

Esvelt KM, Mali P, Braff JL, Moosburner M, Yaung SJ, Church GM (2013): Orthogonal Cas9 proteins for RNA-guided gene regulation and editing. Nat. Methods 10, 1116-1121. https://doi.org/10.1038/nmeth.2681

Finzi D, Blankson J, Siliciano JD, Margolick JB, Chadwick K, Pierson T, Smith K, Lisziewicz J, Lori F, Flexner C, Quinn TC, Chaisson RE, Rosenberg E, Walker B, Gange S, Gallant J, Siliciano RF (1999): Latent infection of CD4+ T cells provides a mechanism for lifelong persistence of HIV-1, even in patients on effective combination therapy. Nat. Med. 5, 512-517. https://doi.org/10.1038/8394

Gabriel R, Lombardo A, Arens A, Miller JC, Genovese P, Kaeppe, C, Nowrouzi A, Bartholomae CC, Wang J, FriedmanG, Holmes, MC, Gregory PD, Glimm H, Schmidt M, Naldini L, von Kalle C (2011): An unbiased genome-wide analysis of zinc-finger nuclease specificity. Nat. Biotechnol. 29, 816-823. https://doi.org/10.1038/nbt.1948

Garneau JE, Dupuis ME, Villion M, Romero DA, Barrangou R, Boyaval P, Fremaux C, Horvath P, Magadan AH, Moineau S (2010): The CRISPR/Cas bacterial immune system cleaves bacteriophage and plasmid DNA. Nature 468, 67-71. https://doi.org/10.1038/nature09523

Gasiunas G, Barrangou R, Horvath P, Siksnys V (2012): Cas9-crRNA ribonucleoprotein complex mediates specific DNA cleavage for adaptive immunity in bacteria. Proc. Natl. Acad. Sci. USA 109, E2579-2586. https://doi.org/10.1073/ pnas. 1208507109

Hahn BH, Shaw GM, Taylor ME, Redfield RR, Markham PD, Salahuddin SZ, Wong-Staal F, Gallo RC, Parks ES, Parks WP (1986): Genetic variation in HTLV-III/LAV over time in patients with AIDS or at risk for AIDS. Science 232, 1548-1553. https://doi.org/10.1126/science.3012778

Han X, Liu Z, Jo MC, Zhang K, Li Y, Zeng Z, Li N, Zu Y, Qin L (2015): CRISPR-Cas9 delivery to hard-to-transfect cells via membrane deformation. Sci. Adv. 1, e1500454. https:// doi.org/10.1126/sciadv.1500454

Hsu PD, Scott DA, Weinstein JA, Ran FA, Konermann S, Agarwala V, Li Y, Fine EJ, Wu X, Shalem O, Cradick TJ, Marraffini LA, Bao G, Zhang F (2013): DNA targeting specificity of RNA-guided Cas9 nucleases. Nat. Biotechnol. 31, 827-832. https://doi.org/10.1038/nbt.2647

Hu W, Kaminski R, Yang F, Zhang Y, Cosentino L, Li F, Luo B, Alvarez-Carbonell D, Garcia-Mesa Y, Karn J, Mo X, Khalili K (2014): RNA-directed gene editing specifically eradicates latent and prevents new HIV-1 infection. Proc. Natl. Acad. Sci. USA 111, 11461-11466. https://doi. org/10.1073/pnas.1405186111 
Kaminski R, Bella R, Yin C, Otte J, Ferrante P, Gendelman HE, Li H, Booze R, Gordon J, Hu W, Khalili K (2016a): Excision of HIV-1 DNA by gene editing: a proof-of-concept in vivo study. Gene Ther. 23, 690-695. https://doi.org/10.1038/ gt.2016.41

Kaminski R, Chen Y, Fischer T, Tedaldi E, Napoli A, Zhang Y, Karn J, Hu W, Khalili K (2016b): Elimination of HIV-1 Genomes from Human T-lymphoid Cells by CRISPR/Cas9 Gene Editing. Sci. Rep. 6, 22555. https://doi.org/10.1038/ $\underline{\operatorname{srep} 28213}$

Kim H, Kim JS (2014): A guide to genome engineering with programmable nucleases. Nat. Rev. Genet. 15, 321-334. https://doi.org/10.1038/nrg3686

Lebbink RJ, de Jong DC, Wolters F, Kruse EM, van Ham PM, Wiertz EJ, Nijhuis M (2017): A combinational CRISPR/ Cas9 gene-editing approach can halt HIV replication and prevent viral escape. Sci. Rep. 7, 41968. https://doi. org/10.1038/srep41968

Leitner T, Korber B, Daniels M, Calef C, Foley B (2005): HIV-1 Subtype and Circulating Recombinant Form (CRF) Reference Sequences, 2005, 41-48. https://doi.org/10.1111/j.00315990.2005.00003.x

Liao HK, Gu Y, Diaz A, Marlett J, Takahashi Y, Li M, Suzuki K, Xu R, Hishida T, Chang CJ, Esteban CR, Young J, Izpisua Belmonte JC (2015): Use of the CRISPR/Cas9 system as an intracellular defense against HIV-1 infection in human cells. Nat. Commun. 6, 6413. https://doi.org/10.1038/ ncomms 7413

Lieber MR (2010): The mechanism of double-strand DNA break repair by the nonhomologous DNA end-joining pathway. Annu. Rev. Biochem. 79, 181-211. https://doi. org/10.1146/annurev.biochem.052308.093131

Maddon PJ, Dalgleish AG, McDougal JS, Clapham PR, Weiss RA, Axel R (1986): The T4 gene encodes the AIDS virus receptor and is expressed in the immune system and the brain. Cell 47, 333-348. https://doi.org/10.1016/0092$\underline{8674(86) 90590-8}$

Makarova KS, Haft DH, Barrangou R, Brouns SJ, Charpentier E, Horvath P, Moineau S, Mojica FJ, Wolf YI, Yakunin AF, van der Oost J, Koonin EV (2011): Evolution and classification of the CRISPR-Cas systems. Nat. Rev. Microbiol. 9, 467-477. https://doi.org/10.1038/nrmicro2577

Manjunath N, Yi G, Dang Y, Shankar P (2013): Newer gene editing technologies toward HIV gene therapy. Viruses 5, 2748-2766. https://doi.org/10.3390/v5112748

Montague TG, Cruz JM, Gagnon JA, Church GM, Valen E (2014): CHOPCHOP: a CRISPR/Cas9 and TALEN web tool for genome editing. Nucleic Acids Res. 42, W401-407. https://doi.org/10.1093/nar/gku410

Nishimasu H, Ran FA, Hsu PD, Konermann S, Shehata SI, Dohmae N, Ishitani R, Zhang F, Nureki O (2014): Crystal structure of Cas 9 in complex with guide RNA and target DNA. Cell 156, 935-949. https://doi.org/10.1016/j.cell.2014.02.001

Palmer S, Josefsson L, Coffin JM (2011): HIV reservoirs and the possibility of a cure for HIV infection. J. Intern. Med. 270, 550560. https://doi.org/10.1111/j.1365-2796.2011.02457.x

Panfil AR, London JA, Green PL, Yoder KE (2018): CRISPR/Cas9 Genome Editing to Disable the Latent HIV-1 Provi- rus. Front Microbiol. 9, 3107. https://doi.org/10.3389/ fmicb.2018.03107

Platt EJ, Bilska M, Kozak SL, Kabat D, Montefiori DC (2009): Evidence that ecotropic murine leukemia virus contamination in TZM-bl cells does not affect the outcome of neutralizing antibody assays with human immunodeficiency virus type 1. J. Virol. 83, 8289-8292. https://doi. org/10.1128/JVI.00709-09

Platt EJ, Wehrly K, Kuhmann SE, Chesebro B, Kabat D (1998): Effects of CCR5 and CD4 cell surface concentrations on infections by macrophagetropic isolates of human immunodeficiency virus type 1. J. Virol. 72, 2855-2864.

Poeschla EM, Wong-Staal F, Looney DJ (1998): Efficient transduction of nondividing human cells by feline immunodeficiency virus lentiviral vectors. Nat. Med. 4, 354-357. https://doi.org/10.1038/nm0398-354

Qu X, Wang P, Ding D, Li L, Wang H, Ma L, Zhou X, Liu S, Lin S, Wang X, Zhang G, Liu S, Liu L, Wang J, Zhang F, Lu D, Zhu H (2013): Zinc-finger-nucleases mediate specific and efficient excision of HIV-1 proviral DNA from infected and latently infected human T cells. Nucleic Acids Res. 41, 7771-7782. https://doi.org/10.1093/nar/gkt571

Ran FA, Cong L, Yan WX, Scott DA, Gootenberg, JS, Kriz AJ, Zetsche B, Shalem O, Wu X, Makarova KS, Koonin EV, Sharp PA, Zhang F (2015): In vivo genome editing using Staphylococcus aureus Cas9. Nature 520, 186-191. https://doi.org/10.1038/nature14299

Rihn SJ, Hughes J, Wilson SJ, Bieniasz PD (2015): Uneven genetic robustness of HIV-1 integrase. J. Virol. 89, 552-567. https://doi.org/10.1128/JVI.02451-14

Rihn SJ, Wilson SJ, Loman NJ, Alim M, Bakker SE, Bhella D, Gifford RJ, Rixon FJ, Bieniasz PD (2013): Extreme genetic fragility of the HIV-1 capsid. PLoS Pathog. 9, e1003461. https://doi.org/10.1371/journal.ppat.1003461

Shalem O, Sanjana NE, Hartenian E, Shi X, Scott DA, Mikkelsen TS, Heckl D, Ebert BL, Root DE, Doench JG, Zhang F (2014): Genome-scale CRISPR-Cas 9 knockout screening in human cells. Science 343, 84-87. https://doi.org/10.1126/ science. 1247005

Siliciano JD, Kajdas J, Finzi D, Quinn TC, Chadwick K, Margolick JB, Kovacs C, Gange SJ, Siliciano RF (2003): Long-term follow-up studies confirm the stability of the latent reservoir for HIV-1 in resting CD4+ T cells. Nat. Med. 9, 727-728. https://doi.org/10.1038/nm880

Sternberg SH, Redding S, Jinek M, Greene EC, Doudna JA (2014): DNA interrogation by the CRISPR RNA-guided endonuclease Cas9. Nature 507, 62-67. https://doi.org/10.1038/ nature13011

Takeuchi Y, McClure MO, Pizzato M (2008): Identification of gammaretroviruses constitutively released from cell lines used for human immunodeficiency virus research. J. Virol. 82, 12585-12588. https://doi.org/10.1128/ JVI.01726-08

Tebas P, Stein D, Tang WW, Frank I, Wang SQ, Lee G, Spratt SK, Surosky RT, Giedlin MA, Nichol G, Holmes MC, Gregory PD, Ando DG, Kalos M, Collman RG, Binder-Scholl G, Plesa G, Hwang WT, Levine BL, June CH (2014): Gene editing of CCR5 in autologous CD4 T cells of persons 
infected with HIV. N. Engl. J. Med. 370, 901-910. https:// doi.org/10.1056/NEJMoa1300662

ter Brake O, von Eije KJ, Berkhout B (2008): Probing the sequence space available for HIV-1 evolution. AIDS 22, 18751877. https://doi.org/10.1097/QAD.0b013e328309efe3

Ueda S, Ebina H, Kanemura Y, Misawa N, Koyanagi Y (2016): AntiHIV-1 potency of the CRISPR/Cas9 system insufficient to fully inhibit viral replication. Microbiol. Immunol. 60, 483-496. https://doi.org/10.1111/1348-0421.12395

von Eije KJ, ter Brake O, Berkhout B (2008): Human immunodeficiency virus type 1 escape is restricted when conserved genome sequences are targeted by RNA interference. J. Virol. 82, 2895-2903. https://doi.org/10.1128/JVI.02035-07

Wain-Hobson S, Sonigo P, Danos O, Cole S, Alizon M (1985): Nucleotide sequence of the AIDS virus, LAV. Cell 40, 9-17. https://doi.org/10.1016/0092-8674(85)90303-4

Wain-Hobson S, Vartanian JP, Henry M, Chenciner N, Cheynier R, Delassus S, Martins LP, Sala M, Nugeyre MT, Guetard D et al. (1991): LAV revisited: origins of the early HIV1 isolates from Institut Pasteur. Science 252, 961-965. https://doi.org/10.1126/science.2035026

Wang D, Mou H, Li S, Li Y, Hough S, Tran K, Li J, Yin H, Anderson DG, Sontheimer EJ, Weng Z, Gao G, Xue W (2015a): Adenovirus-Mediated Somatic Genome Editing of Pten by CRISPR/Cas9 in Mouse Liver in Spite of Cas9-Specific Immune Responses. Hum. Gene Ther. 26, 432-442. https://doi.org/10.1089/hum.2015.087

Wang G, Zhao N, Berkhout B, Das AT (2016a): A Combinatorial CRISPR-Cas9 Attack on HIV-1 DNA Extinguishes All Infectious Provirus in Infected T Cell Cultures. Cell. Rep. 17, 2819-2826. https://doi.org/10.1016/j.celrep.2016.11.057

Wang G, Zhao N, Berkhout B, Das AT (2016b): CRISPR-Cas9 Can Inhibit HIV-1 Replication but NHEJ Repair Facilitates Virus Escape. Mol. Ther. 24, 522-526. https://doi. org $/ 10.1038 / \mathrm{mt} .2016 .24$

Wang H, Yang H, Shivalila CS, Dawlaty MM, Cheng AW, Zhang F, Jaenisch R (2013): One-step generation of mice carrying mutations in multiple genes by CRISPR/Cas-mediated genome engineering. Cell 153, 910-918. https://doi. org/10.1016/j.cell.2013.04.025

Wang X, Wang Y, Wu X, Wang J, Wang Y, Qiu Z, Chang T, Huang $\mathrm{H}$, Lin RJ, Yee JK (2015b): Unbiased detection of off-target cleavage by CRISPR-Cas9 and TALENs using integrasedefective lentiviral vectors. Nat. Biotechnol. 33, 175-178. https://doi.org/10.1038/nbt.3127
Wang Z, Pan Q, Gendron P, Zhu W, Guo F, Cen S, Wainberg MA, Liang C (2016c): CRISPR/Cas9-Derived Mutations Both Inhibit HIV-1 Replication and Accelerate Viral Escape. Cell. Rep. 15, 481-489. https://doi.org/10.1016/j.celrep.2016.03.042

Wei X, Decker JM, Liu H, Zhang Z, Arani RB, Kilby JM, Saag MS, Wu X, Shaw GM, Kappes JC (2002): Emergence of resistant human immunodeficiency virus type 1 in patients receiving fusion inhibitor (T-20) monotherapy. Antimicrob. Agents Chemother. 46, 1896-1905. https:// doi.org/10.1128/AAC.46.6.1896-1905.2002

Yin C, Zhang T, Li F, Yang F, Putatunda R, Young WB, Khalili K, Hu W, Zhang Y (2016): Functional screening of guide RNAs targeting the regulatory and structural HIV-1 viral genome for a cure of AIDS. AIDS 30, 1163-1174. https:// doi.org/10.1097/QAD.0000000000001079

Yin C, Zhang T, Qu X, Zhang Y, Putatunda R, Xiao X, Li F, Xiao W, Zhao H, Dai S, Qin X, Mo X, Young WB, Khalili K, Hu W (2017): In Vivo Excision of HIV-1 Provirus by saCas9 and Multiplex Single-Guide RNAs in Animal Models. Mol. Ther. 25, 1168-1186. https://doi.org/10.1016/j. ymthe.2017.03.012

Yoder KE, Bundschuh R (2016): Host Double Strand Break Repair Generates HIV-1 Strains Resistant to CRISPR/Cas9. Sci. Rep. 6, 29530. https://doi.org/10.1038/srep29530

Zanini F, Puller V, Brodin J, Albert J, Neher RA (2017): In vivo mutation rates and the landscape of fitness costs of HIV-1. Virus Evol. 3, vex003. https://doi.org/10.1093/ve/vex003

Zetsche B, Gootenberg JS, Abudayyeh OO, Slaymaker IM, Makarova KS, Essletzbichler P, Volz SE, Joung J, van der Oost J, Regev A, Koonin EV, Zhang F (2015): Cpf1 is a single RNA-guided endonuclease of a class 2 CRISPRCas system. Cell 163, 759-771. https://doi.org/10.1016/j. cell.2015.09.038

Zhou H, Xu M, Huang Q, Gates AT, Zhang XD, Castle JC, Stec E, Ferrer M, Strulovici B, Hazuda DJ, Espeseth AS (2008): Genome-scale RNAi screen for host factors required for HIV replication. Cell Host Microbe 4, 495-504. https:// doi.org/10.1016/j.chom.2008.10.004

Zhu W, Lei R, Le Duff Y, Li J, Guo F, Wainberg MA, Liang C (2015): The CRISPR/Cas9 system inactivates latent HIV-1 proviral DNA. Retrovirology 12, 22. https://doi.org/10.1186/ $\underline{\text { s12977-015-0150-Z }}$ 


\title{
Supplementary information
}

\section{A CRISPR/Cas9 library to map the HIV-1 provirus genetic fitness}

\author{
K. E. YODER
}

The Ohio State University College of Medicine, Cancer Biology and Genetics, 460 West $12^{\text {th }}$ Ave. Room 1006, Columbus, $\mathrm{OH} 43210$ USA

Received June 27, 2018; accepted September 27, 2018

E-mail: yoder.176@osu.edu; phone: +1-(614)-688-2106. 


\begin{tabular}{|c|c|c|c|c|}
\hline LAI Target sequence & 5' end of gRNA target site & Strand & \% GC content & HIV-1 target \\
\hline $\begin{array}{l}\text { CAAGGATATCTTGTCTTCGTTGG } \\
\end{array}$ & sequence: 20,9152 & - & 40 & LTR U3 NEF \\
\hline GACAAGATATCCTTGATCTGTGG & sequence: 28,9160 & + & 40 & LTR U3 NEF \\
\hline TGTGGTAGATCCACAGATCAAGG & sequence: 38,9170 & - & 45 & LTR U3 NEF \\
\hline CTGTGGATCTACCACACACAAGG & sequence: 45,9177 & + & 50 & LTR U3 NEF \\
\hline CAGGGAAGTAGCCTTGTGTGTGG & sequence:56, 9188 & - & 55 & LTR U3 NEF \\
\hline CACAAGGCTACTTCCCTGATTGG & sequence:61, 9193 & + & 50 & LTR U3 NEF \\
\hline GTGTGTAGTTCTGCCAATCAGGG & sequence:74, 9206 & - & 45 & LTR U3 NEF \\
\hline GGTGTGTAGTTCTGCCAATCAGG & sequence: 75,9207 & - & 50 & LTR U3 NEF \\
\hline GATTGGCAGAACTACACACCAGG & sequence:78, 9210 & + & 50 & LTR U3 NEF \\
\hline ATTGGCAGAACTACACACCAGGG & sequence:79, 9211 & + & 45 & LTR U3 NEF \\
\hline CAGAACTACACACCAGGGCCAGG & sequence: 84,9216 & + & 60 & LTR U3 NEF \\
\hline AGAACTACACACCAGGGCCAGGG & sequence:85, 9217 & + & 55 & LTR U3 NEF \\
\hline GAACTACACACCAGGGCCAGGGG & sequence:86, 9218 & + & 60 & LTR U3 NEF \\
\hline GGATATCTGACCCCTGGCCCTGG & sequence:96, 9228 & - & 65 & LTR U3 NEF \\
\hline GTCAGTGGATATCTGACCCCTGG & sequence:102, 9234 & - & 55 & LTR U3 NEF \\
\hline GTCAGATATCCACTGACCTTTGG & sequence:108, 9240 & + & 45 & LTR U3 NEF \\
\hline GATATCCACTGACCTTTGGATGG & sequence:112, 9244 & + & 45 & LTR U3 NEF \\
\hline TAGCTTGTAGCACCATCCAAAGG & sequence:124, 9256 & - & 45 & LTR U3 NEF \\
\hline AGTACCAGTTGAGCCAGATAAGG & sequence:146, 9278 & + & 45 & LTR U3 NEF \\
\hline TCTACCTTATCTGGCTCAACTGG & sequence:150, 9282 & - & 45 & LTR U3 NEF \\
\hline TGAGCCAGATAAGGTAGAAGAGG & sequence:155, 9287 & + & 45 & LTR U3 NEF \\
\hline TTGGCCTCTTCTACCTTATCTGG & sequence:159, 9291 & - & 45 & LTR U3 NEF \\
\hline AAGGTAGAAGAGGCCAATAAAGG & sequence:165, 9297 & + & 40 & LTR U3 NEF \\
\hline GCTGGTGTTCTCTCCTTTATTGG & sequence: 178,9310 & - & 45 & LTR U3 NEF \\
\hline GCTCACAGGGTGTAACAAGCTGG & sequence:196, 9328 & - & 55 & LTR U3 NEF \\
\hline TTACACCCTGTGAGCCTGCATGG & sequence:204, 9336 & + & 55 & LTR U3 NEF \\
\hline СCCTGTGAGCCTGCATGGAATGG & sequence:209, 9341 & + & 60 & LTR U3 NEF \\
\hline TCCATTCCATGCAGGCTCACAGG & sequence:210, 9342 & - & 55 & LTR U3 NEF \\
\hline CAGGGTCATCCATTCCATGCAGG & sequence: 218,9350 & - & 55 & LTR U3 NEF \\
\hline АСTCTAACACTTCTCTCTCAGGG & sequence:236, 9368 & - & 40 & LTR U3 NEF \\
\hline CTGAGAGAGAAGTGTTAGAGTGG & sequence:238, 9370 & + & 45 & LTR U3 NEF \\
\hline AGAGAGAAGTGTTAGAGTGGAGG & sequence:241, 9373 & + & 45 & LTR U3 NEF \\
\hline CCGCCTAGCATTTCATCACGTGG & sequence: 272,9404 & + & 55 & LTR U3 NEF \\
\hline GGGCCACGTGATGAAATGCTAGG & sequence:275, 9407 & - & 55 & LTR U3 NEF \\
\hline CGTGGCCCGAGAGCTGCATCCGG & sequence:290, 9422 & + & 70 & LTR U3 NEF \\
\hline GTACTCCGGATGCAGCTCTCGGG & sequence:295, 9427 & - & 60 & LTR U3 NEF \\
\hline AGTACTCCGGATGCAGCTCTCGG & sequence:296, 9428 & - & 55 & LTR U3 NEF \\
\hline CAGCAGTTCTTGAAGTACTCCGG & sequence: 309,9441 & - & 45 & LTR U3 NEF \\
\hline CTGACATCGAGCTTGCTACAAGG & sequence:329, 9461 & + & 50 & LTR U3 \\
\hline TGACATCGAGCTTGCTACAAGGG & sequence:330, 9462 & + & 45 & LTR U3 \\
\hline TGCTACAAGGGACTTTCCGCTGG & sequence:342, 9474 & + & 55 & LTR U3 \\
\hline GCTACAAGGGACTTTCCGCTGGG & sequence: 343,9475 & + & 55 & LTR U3 \\
\hline CTACAAGGGACTTTCCGCTGGGG & sequence:344, 9476 & + & 55 & LTR U3 \\
\hline CTTTCCGCTGGGGACTTTCCAGG & sequence:354, 9486 & + & 60 & LTR U3 \\
\hline TTTCCGCTGGGGACTTTCCAGGG & sequence:355, 9487 & + & 55 & LTR U3 \\
\hline CCGCTGGGGACTTTCCAGGGAGG & sequence: 358,9490 & + & 70 & LTR U3 \\
\hline GGGGACTTTCCAGGGAGGCGTGG & sequence:363, 9495 & + & 70 & LTR U3 \\
\hline CTTTCCAGGGAGGCGTGGCCTGG & sequence: 368,9500 & + & 70 & LTR U3 \\
\hline TTTCCAGGGAGGCGTGGCCTGGG & sequence:369, 9501 & + & 65 & LTR U3 \\
\hline TGCTTATATGCAGCATCTGAGGG & sequence:412, 9544 & - & 40 & LTR U3 \\
\hline CTGCTTATATGCAGCATCTGAGG & sequence: 413,9545 & - & 45 & LTR U3 \\
\hline CAGCTGCTTTTTGCCTGTACTGG & sequence: 433,9565 & + & 50 & LTR U3 \\
\hline AGCTGCTTTTTGGCTGTACTGGG & sequence:434, 9566 & + & 45 & LTR U3 \\
\hline TTGCCTGTACTGGGTCTCTCTGG & sequence: 443,9575 & + & 55 & LTR U3 \\
\hline TAACCAGAGAGACCCAGTACAGG & sequence: 446,9578 & - & 50 & LTR U3 \\
\hline GGTTAGACCAGATTTGAGCCTGG & sequence:464, 9596 & + & 50 & LTR R \\
\hline GTTAGACCAGATTTGAGCCTGGG & sequence: 465,9597 & + & 45 & LTR R \\
\hline AGAGCTCCCAGGCTCAAATCTGG & sequence:471, 9603 & - & 55 & LTR R \\
\hline ATTTGAGCCTGGGAGCTCTCTGG & sequence: 475,9607 & + & 55 & LTR R \\
\hline TAGTTAGCCAGAGAGCTCCCAGG & sequence:482, 9614 & - & 55 & LTR R \\
\hline TGGGAGCTCTCTGGCTAACTAGG & sequence:484, 9616 & + & 55 & LTR R \\
\hline GGGAGCTCTCTGGCTAACTAGGG & sequence:485, 9617 & + & 55 & LTR R \\
\hline CTTTATTGAGGCTTAAGCAGTGG & sequence:511, 9643 & - & 40 & LTR R \\
\hline ACTCAAGGCAAGCTTTATTGAGG & sequence:523, 9655 & - & 40 & LTR R \\
\hline CACACTACTTGAAGCACTCAAGG & sequence:538, 9670 & - & 45 & LTR U5 \\
\hline GCCCGTCTGTTGTGTGACTCTGG & sequence:562, 9694 & + & 60 & LTR U5 \\
\hline ACCAGAGTCACACAACAGACGGG & sequence:563, 9695 & - & 50 & LTR U5 \\
\hline TACCAGAGTCACACAACAGACGG & sequence:564, 9696 & - & 45 & LTR U5 \\
\hline CACTGACTAAAAGGGTCTGAGGG & sequence:596, 9728 & - & 45 & LTR U5 \\
\hline ACACTGACTAAAAGGGTCTGAGG & sequence:597, 9729 & - & 45 & LTR U5 \\
\hline
\end{tabular}


TCAGACCCTTTTAGTCAGTGTGG GTGTGGAAAATCTCTAGCAGTGG TCTAGCAGTGGCGCCCGAACAGG CTAGCAGTGGCGCCCGAACAGGG TCGCTTTCAAGTCCCTGTTCGGG TTCGCTTTCAAGTCCCTGTTCGG AACAGGGACTTGAAAGCGAAAGG ACAGGGACTTGAAAGCGAAAGGG GAAAGCGAAAGGGAAACCAGAGG CTGCGTCGAGAGAGCTCCTCTGG CAGAGGAGCTCTCTCGACGCAGG AGCTCTCTCGACGCAGGACTCGG TCGGCTTGCTGAAGCGCGCACGG GCTGAAGCGCGCACGGCAAGAGG AAGAGGCGAGGGGAGGCGACTGG CCAAAAATTTTGACTAGCGGAGG TTTGACTAGCGGAGGCTAGAAGG GAGGCTAGAAGGAGAGAGATGGG GCGAGAGCGTCAGTATTAAGCGG CGAGAGCGTCAGTATTAAGCGGG GAGAGCGTCAGTATTAAGCGGGG AGAGCGTCAGTATTAAGCGGGGG GCGGGGGAGAATTAGATCGATGG CGGGGGAGAATTAGATCGATGGG GAAAAAATTCGGTTAAGGCCAGG AAAATTCGGTTAAGGCCAGGGGG AACATATAGTATGGGCAAGCAGG ACATATAGTATGGGCAAGCAGGG GAACGATTCGCAGTTAATCCTGG TCTGATGTTTCTAACAGGCCAGG GGCCTGTTAGAAACATCAGAAGG AGCCTTCTGATGTTTCTAACAGG AGAAGGCTGTAGACAAATACTGG GAAGGCTGTAGACAAATACTGGG CTACAACCATCCCTTCAGACAGG TCTGATCCTGTCTGAAGGGATGG TTCTTCTGATCCTGTCTGAAGGG GTTCTTCTGATCCTGTCTGAAGG CCCTCTATTGTGTGCATCAAAGG TCCTTTGATGCACACAATAGAGG GGAAGCTTTAGACAAGATAGAGG TGACACAGGACACAGCAGCCAGG TAGGGTAATTTTGGCTGACCTGG CCCTATAGTGCAGAACATCCAGG CCTATAGTGCAGAACATCCAGGG CTATAGTGCAGAACATCCAGGGG GCAGAACATCCAGGGGCAAATGG CCAGGGGCAAATGGTACATCAGG GAAAACATGGGTATCACTTCTGG GGTGTTTAAATCTTGTGGGGTGG AAACACCATGCTAAACACAGTGG AACACCATGCTAAACACAGTGGG ACACCATGCTAAACACAGTGGGG CACCATGCTAAACACAGTGGGGG ACCATGCTAAACACAGTGGGGGG TCCCCCCACTGTGTTTAGCATGG TTCTGCAGCTTCCTCATTGATGG TCAATGAGGAAGCTGCAGAATGG CAATGAGGAAGCTGCAGAATGGG AGAGTGCATCCAGTGCATGCAGG GAGTGCATCCAGTGCATGCAGGG GCAATAGGCCCTGCATGCACTGG CATGCAGGGCCTATTGCACCAGG CTCATCTGGCCTGGTGCAATAGG CTTGGTTCTCTCATCTGGCCTGG CAGGCCAGATGAGAGAACCAAGG AGGCCAGATGAGAGAACCAAGGG GGCCAGATGAGAGAACCAAGGGG TTCCCCTTGGTTCTCTCATCTGG CCAAGGGGAAGTGACATAGCAGG AGGAACTACTAGTACCCTTCAGG sequence:599, 9731

sequence: 616

sequence: 628

sequence:629

sequence:641

sequence: 642

sequence:645

sequence: 646

sequence: 656

sequence: 672

sequence: 673

sequence: 679

sequence:698

sequence:705

sequence: 722

sequence:753

sequence:761

sequence:772

sequence:796

sequence:797

sequence:798

sequence:799

sequence:815

sequence: 816

sequence:838

sequence:841

sequence:884

sequence:885

sequence:913

sequence:931

sequence:934

sequence:936

sequence:951

sequence:952

sequence:979

sequence:985

sequence:989

sequence:990

sequence: 1040

sequence:1041

sequence:1083

sequence:1149

sequence:1167

sequence:1185

sequence:1186

sequence:1187

sequence:1194

sequence:1203

sequence:1285

sequence: 1325

sequence:1341

sequence:1342

sequence: 1343

sequence:1344

sequence:1345

sequence:1346

sequence: 1400

sequence:1403

sequence:1404

sequence: 1429

sequence: 1430

sequence:1438

sequence: 1444

sequence: 1453

sequence:1462

sequence:1463

sequence:1464

sequence:1465

sequence:1467

sequence: 1480

sequence: 1500
LTR U5

LTR U5

LTR U5

LTR U5

PBS

PBS

PBS

PBS

DIS

DIS

DIS

PSI

PSI

PSI

GAG

GAG

GAG

GAG

GAG

GAG

GAG

GAG

GAG

GAG

GAG

GAG

GAG

GAG

GAG

GAG

GAG

GAG

GAG

GAG

GAG

GAG

GAG

GAG

GAG

GAG

GAG

GAG

GAG

GAG

GAG

GAG

GAG

GAG

GAG

GAG

GAG

GAG

GAG

GAG

GAG

GAG

GAG

GAG

GAG

GAG

GAG

GAG

GAG

GAG

GAG

GAG

GAG 
AGTACCCTTCAGGAACAAATAGG CCCTTCAGGAACAAATAGGATGG TCCATCCTATTTGTTCCTGAAGG AATAATCCACCTATCCCAGTAGG ATTTCTCCTACTGGGATAGGTGG GTATAGCCCTACCAGCATTCTGG TTATGTCCAGAATGCTGGTAGGG CTTATGTCCAGAATGCTGGTAGG AGCATTCTGGACATAAGACAAGG CGGTCTACATAGTCTCTAAAGGG CCTTTAGAGACTATGTAGACCGG AAGAGCCGAGCAAGCTTCACAGG AGCCGAGCAAGCTTCACAGGAGG TACCTCCTGTGAAGCTTGCTCGG TTGGATGACAGAAACCTTGTTGG GTTCGCATTTTGGACCAACAAGG TACAATCTGGGTTCGCATTTTGG ATTTCTTCTAGTGTAGCTGCTGG AGAAATGATGACAGCATGTCAGG GAAATGATGACAGCATGTCAGGG GATGACAGCATGTCAGGGAGTGG ATGACAGCATGTCAGGGAGTGGG ACAGCATGTCAGGGAGTGGGAGG TGTCAGGGAGTGGGAGGACCCGG AGTGGGAGGACCCGGCCATAAGG AAAACTCTTGCCTTATGGCCGGG CAAAACTCTTGCCTTATGGCCGG CGGCCATAAGGCAAGAGTTTTGG CAGCCAAAACTCTTGCCTTATGG GCTACCATAATGATGCAAAGAGG GGCACATAGCCAGAAATTGCAGG GCACATAGCCAGAAATTGCAGGG CCAGAAATTGCAGGGCCCCTAGG TTGCAGGGCCCCTAGGAAAAAGG TGCAGGGCCCCTAGGAAAAAGGG CCCCTAGGAAAAAGGGCTGTTGG TCCAACAGCCCTTTTTCCTAGGG TTCCAACAGCCCTTTTTCCTAGG AAAAAGGGCTGTTGGAAATGTGG GGGCTGTTGGAAATGTGGAAAGG TGTTGGAAATGTGGAAAGGAAGG GAAAGATTGTACTGAGAGACAGG GAAGATCTGGCCTTCCTACAAGG AAGATCTGGCCTTCCTACAAGGG TCTGGCCTTCCTACAAGGGAAGG CCTTCCTACAAGGGAAGGCCAGG CTTCCTACAAGGGAAGGCCAGGG ATTCCCTGGCCTTCCCTTGTAGG CTGCTCTGAAGAAAATTCCCTGG AATGGTGGGGCTGTTGGCTCTGG TGAAGAAATGGTGGGGCTGTTGG TCTGCTCTGAAGAAATGGTGGGG GTCTGCTCTGAAGAAATGGTGGG GGTCTGCTCTGAAGAAATGGTGG TCTGGTCTGCTCTGAAGAAATGG TCTGGTGGGGCTGTTGGCTCTGG CTCTCTTCTGGTGGGGCTGTTGG CCCCACCAGAAGAGAGCTTCAGG ACCTGAAGCTCTCTTCTGGTGGG GACCTGAAGCTCTCTTCTGGTGG CCAGAAGAGAGCTTCAGGTCTGG CAGAAGAGAGCTTCAGGTCTGGG AGAAGAGAGCTTCAGGTCTGGGG AACAACTCCCTCTCAGAAGCAGG ATCGGCTCCTGCTTCTGAGAGGG TATCGGCTCCTGCTTCTGAGAGG GAAGCAGGAGCCGATAGACAAGG GGATACAGTTCCTTGTCTATCGG AGTGATCTGAGGGAAGTTAAAGG ACTTCCCTCAGATCACTCTTTGG GTTGCCAAAGAGTGATCTGAGGG sequence: 1510

sequence: 1514

sequence: 1515

sequence: 1543

sequence: 1549

sequence: 1617

sequence: 1623

sequence:1624

sequence: 1630

sequence: 1663

sequence:1664

sequence: 1701

sequence: 1704

sequence: 1706

sequence: 1734

sequence: 1748

sequence: 1758

sequence:1804

sequence: 1821

sequence: 1822

sequence: 1827

sequence:1828

sequence:1831

sequence: 1837

sequence: 1845

sequence:1855

sequence:1856

sequence:1857

sequence: 1860

sequence:1909

sequence:1985

sequence:1986

sequence:1994

sequence:2001

sequence: 2002

sequence:2009

sequence: 2010

sequence:2011

sequence: 2017

sequence: 2022

sequence:2026

sequence: 2058

sequence: 2094

sequence:2095

sequence:2099

sequence:2104

sequence:2105

sequence: 2108

sequence: 2122

sequence:2146

sequence: 2152

sequence: 2159

sequence: 2160

sequence:2161

sequence: 2164

sequence: 2182

sequence: 2188

sequence:2195

sequence: 2196

sequence: 2197

sequence: 2200

sequence:2201

sequence: 2202

sequence: 2232

sequence:2239

sequence: 2240

sequence: 2247

sequence: 2257

sequence: 2278

sequence: 2284

sequence:2288
GAG

GAG

GAG

GAG

GAG

GAG

GAG

GAG

GAG

GAG

GAG

GAG

GAG

GAG

GAG

GAG

GAG

GAG

GAG

GAG

GAG

GAG

GAG

GAG

GAG

GAG

GAG

GAG

GAG

GAG

GAG

GAG

GAG

GAG

GAG

GAG

GAG

GAG

GAG

GAG

GAG

GAG

GAG POL

GAG POL

GAG POL

GAG POL

GAG POL

GAG POL

GAG POL

GAG POL

GAG POL

GAG POL

GAG POL

GAG POL

GAG POL

GAG POL

GAG POL

GAG POL

GAG POL

GAG POL

GAG POL

GAG POL

GAG POL

GAG POL

GAG POL

GAG POL

GAG POL

GAG POL

GAG POL

GAG POL

GAG POL 
CGTTGCCAAAGAGTGATCTGAGG CCCCTCGTCACAATAAAGATAGG CCCTCGTCACAATAAAGATAGGG CCTCGTCACAATAAAGATAGGGG CTCGTCACAATAAAGATAGGGGG TCGTCACAATAAAGATAGGGGGG AAAGATAGGGGGGCAACTAAAGG AAATGAGTTTGCCAGGAAGATGG CCAAAAATGATAGGGGGAATTGG AAAATGATAGGGGGAATTGGAGG CCTACACCTGTCAACATAATTGG ACTTTTGGGCCATCCATTCCTGG GACTTCTGGGAAGTTCAATTAGG TTAGGAATACCACATCCCGCAGG TAGGAATACCACATCCCGCAGGG TTTTTTAACCCTGCGGGATGTGG ATCAGTAACAGTACTGGATGTGG TCAGTAACAGTACTGGATGTGGG TTCCCTTAGATGAAGACTTCAGG CTTCCTGAAGTCTTCATCTAAGG TCAGTACAATGTGCTTCCACAGG CAGTACAATGTGCTTCCACAGGG ACAATGTGCTTCCACAGGGATGG GTGCTTCCACAGGGATGGAAAGG GGTGATCCTTTCCATCCCTGTGG AGCTGAGACAACATCTGTTGAGG TGAGACAACATCTGTTGAGGTGG GAGACAACATCTGTTGAGGTGGG AGACAACATCTGTTGAGGTGGGG AGAAAGAACCTCCATTCCTTTGG AGAACCTCCATTCCTTTGGATGG GAACCTCCATTCCTTTGGATGGG TCATAACCCATCCAAAGGAATGG GGAGTTCATAACCCATCCAAAGG GTACTGTCCATTTATCAGGATGG GGCTGTACTGTCCATTTATCAGG TCTTTTTCTGGCAGCACTATAGG TGCTGCCAGAAAAAGACAGCTGG ACAGTCCAGCTGTCTTTTTCTGG TGGGCAAGTCAGATTTACCCAGG GGGCAAGTCAGATTTACCCAGGG TTAGTGCTTTGGTTCCTCTAAGG AGCTCTGCTTCTTCTGTTAGTGG AGAAGAAGCAGAGCTAGAACTGG TAGCAGAAATACAGAAGCAGGGG GAAATACAGAAGCAGGGGCAAGG AGAAGCAGGGGCAAGGCCAATGG CAGGAAAATATGCAAGAACGAGG AGGAAACATGGGAAACATGGTGG AAACATGGTGGACAGAGTATTGG CAGAGTATTGGCAAGCCACCTGG AAGCCACCTGGATTCCTGAGTGG AGCCACCTGGATTCCTGAGTGGG AAACTCCCACTCAGGAATCCAGG GTATTGACAAACTCCCACTCAGG CCCCTCCTTTAGTGAAATTATGG CTATGGGTTCTTTCTCTAACTGG AACGTTTCTGCTCCTACTATGGG GAACGTTTCTGCTCCTACTATGG GCAGAAACGTTCTATGTAGATGG ATGTAGATGGGGCAGCTAGCAGG TGTAGATGGGGCAGCTAGCAGGG GCTAGCAGGGAGACTAAATTAGG AGCAATTCATCTAGCTTTGCAGG TCATCTAGCTTTGCAGGATTCGG CATCTAGCTTTGCAGGATTCGGG AGGAAAAGGTCTATCTGGCATGG GGAAAAGGTCTATCTGGCATGGG CCAGCACACAAAGGAATTGGAGG AATTAGTCAGTGCTGGAATCAGG TCACAGTAATTGGAGAGCAATGG sequence:2289

sequence: 2313

sequence: 2314

sequence: 2315

sequence: 2316

sequence: 2317

sequence: 2327

sequence:2392

sequence: 2418

sequence: 2421

sequence: 2523

sequence: 2625

sequence: 2841

sequence: 2859

sequence: 2860

sequence: 2868

sequence: 2897

sequence: 2898

sequence: 2938

sequence:2941

sequence: 3017

sequence:3018

sequence:3022

sequence: 3027

sequence:3033

sequence:3196

sequence:3199

sequence: 3200

sequence:3201

sequence: 3250

sequence: 3254

sequence:3255

sequence:3261

sequence: 3266

sequence: 3287

sequence:3291

sequence:3312

sequence:3319

sequence:3324

sequence: 3381

sequence:3382

sequence:3431

sequence: 3465

sequence: 3473

sequence:3562

sequence: 3567

sequence:3574

sequence:3637

sequence:3769

sequence:3781

sequence:3793

sequence:3805

sequence:3806

sequence:3811

sequence:3819

sequence:3841

sequence:3866

sequence: 3882

sequence:3883

sequence:3894

sequence:3907

sequence: 3908

sequence:3921

sequence: 4025

sequence:4031

sequence:4032

sequence: 4168

sequence:4169

sequence:4194

sequence:4234

sequence:4310
GAG POL

GAG POL

GAG POL

GAG POL

GAG POL

GAG POL

GAG POL

POL

POL

POL

POL

POL

POL

POL

POL

POL

POL

POL

$\mathrm{POL}$

$\mathrm{POL}$

POL

$\mathrm{POL}$

POL

POL

POL

$\mathrm{POL}$

POL

POL

$\mathrm{POL}$

POL

$\mathrm{POL}$

$\mathrm{POL}$

POL

$\mathrm{POL}$

POL

POL

POL

POL

POL

POL

$\mathrm{POL}$

POL

POL

$\mathrm{POL}$

$\mathrm{POL}$

$\mathrm{POL}$

POL

$\mathrm{POL}$

POL

POL

$\mathrm{POL}$

POL

$\mathrm{POL}$

$\mathrm{POL}$

POL

$\mathrm{POL}$

$\mathrm{POL}$

POL

POL

POL

POL

POL

$\mathrm{POL}$

POL

POL

$\mathrm{POL}$

POL

POL

$\mathrm{POL}$

$\mathrm{POL}$

POL 
CTTTTGCTACTACAGGTGGCAGG TAGCTGACATTTATCACAGCTGG CTAAAAGGAGAAGCCATGCATGG GGACAAGTAGACTGTAGTCCAGG TAGACTGTAGTCCAGGAATATGG CAATCTAGTTGCCATATTCCTGG TGGCTACATGAACTGCTACCAGG GTAGCAGTTCATGTAGCCAGTGG GAAGTTATTCCAGCAGAAACAGG TATTCCAGCAGAAACAGGGCAGG CAGCAATTTCACCAGTACTACGG TTTCACCAGTACTACGGTTAAGG GGCGGCCTTAACCGTAGTACTGG CTACGGTTAAGGCCGCCTGTTGG CGGTTAAGGCCGCCTGTTGGTGG GGTTAAGGCCGCCTGTTGGTGGG TAAGGCCGCCTGTTGGTGGGCGG AAGGCCGCCTGTTGGTGGGCGGG CTTGATTCCCGCCCACCAACAGG TTGGTGGGCGGGAATCAAGCAGG CCCTACAATCCCCAAAGTCAAGG TCCTTGACTTTGGGGATTGTAGG TCTACTACTCCTTGACTTTGGGG TATAGGCCAGGTAAGAGATCAGG GTTCAGCCTGATCTCTTACCTGG TAAAAGAAAAGGGGGGATTGGGG AAAAGAAAAGGGGGGATTGGGGG AAAGAAAAGGGGGGATTGGGGGG GGGATTGGGGGGTACAGTGCAGG GGATTGGGGGGTACAGTGCAGGG GATTGGGGGGTACAGTGCAGGGG GATTCCCGCCCACCAACAGGCGG GGGACAGCAGAGATCCACTTTGG AGCAGAGATCCACTTTGGAAAGG TTTGCTGGTCCTTTCCAAAGTGG AAGGACCAGCAAAGCTCCTCTGG CCAGCAAAGCTCCTCTGGAAAGG AAGCTCCTCTGGAAAGGTGAAGG AGCTCCTCTGGAAAGGTGAAGGG GCTCCTCTGGAAAGGTGAAGGGG CTGCCCCTTCACCTTTCCAGAGG GATTATGGAAAACAGATGGCAGG TGATTGTGTGGCAAGTAGACAGG TGTGGCAAGTAGACAGGATGAGG ACAGGATGAGGATTAGAACATGG TGTATGTTTCAGGGAAAGCTAGG GTATGTTTCAGGGAAAGCTAGGG TATGTTTCAGGGAAAGCTAGGGG TTTCAGGGAAAGCTAGGGGATGG TCAGAAGTACACATCCCACTAGG CAGAAGTACACATCCCACTAGGG AGAAGTACACATCCCACTAGGGG CCCACTAGGGGATGCTAGATTGG ACCAATCTAGCATCCCCTAGTGG ACATATTGGGGTCTGCATACAGG TGCATACAGGAGAAAGAGACTGG AGGAGAAAGAGACTGGCATCTGG GGAGAAAGAGACTGGCATCTGGG AAGAGACTGGCATCTGGGTCAGG AGAGACTGGCATCTGGGTCAGGG GTCAGGGAGTCTCCATAGAATGG AGGGAGTCTCCATAGAATGGAGG TTAGTTGGTCTGCTAGTTCAGGG ATTAGTTGGTCTGCTAGTTCAGG TAGGACATATAGTTAGCCCTAGG CTGCTTGATATTCACACCTAGGG CCTAGGTGTGAATATCAAGCAGG CAAGCAGGACATAACAAGGTAGG GGTAGGATCTCTACAATACTTGG TTCGTAACACTAGGCAAAGGTGG AGTTTCGTAACACTAGGCAAAGG sequence:4346

sequence: 4378

sequence: 4398

sequence:4419

sequence: 4426

sequence:4437

sequence: 4484

sequence: 4488

sequence: 4524

sequence: 4529

sequence:4619

sequence:4625

sequence: 4630

sequence:4636

sequence:4639

sequence: 4640

sequence: 4643

sequence: 4644

sequence:4651

sequence:4655

sequence:4689

sequence: 4690

sequence:4698

sequence: 4748

sequence:4754

sequence:4820

sequence:4821

sequence:4822

sequence:4833

sequence:4834

sequence:4835

sequence:4648

sequence: 4948

sequence: 4953

sequence:4962

sequence:4972

sequence:4977

sequence:4983

sequence:4984

sequence:4985

sequence:4988

sequence:5073

sequence:5099

sequence:5105

sequence:5117

sequence:5162

sequence:5163

sequence:5164

sequence:5168

sequence:5233

sequence:5234

sequence:5235

sequence:5247

sequence:5248

sequence:5278

sequence:5291

sequence:5298

sequence:5299

sequence:5304

sequence:5305

sequence:5321

sequence:5324

sequence:5373

sequence:5374

sequence:5450

sequence:5466

sequence:5467

sequence:5482

sequence:5499

sequence:5557

sequence:5560
$50 \quad$ POL

$40 \quad \mathrm{POL}$

$45 \quad \mathrm{POL}$

$50 \quad \mathrm{POL}$

$40 \quad$ POL

$40 \quad \mathrm{POL}$

50 POL

$50 \quad$ POL

$40 \quad \mathrm{POL}$

$50 \quad \mathrm{POL}$

$40 \quad \mathrm{POL}$

$40 \quad \mathrm{POL}$

$60 \quad \mathrm{POL}$

$60 \quad \mathrm{POL}$

$65 \quad \mathrm{POL}$

$60 \quad \mathrm{POL}$

$65 \quad \mathrm{POL}$

$70 \quad \mathrm{POL}$

$60 \quad \mathrm{POL}$

$60 \quad \mathrm{POL}$

50 POL

$45 \quad \mathrm{POL}$

$40 \quad \mathrm{POL}$

$45 \quad \mathrm{POL}$

$50 \quad \mathrm{POL}$

$40 \quad$ POL

$45 \quad \mathrm{POL}$

$50 \quad \mathrm{POL}$

$65 \quad \mathrm{POL}$

$60 \quad \mathrm{POL}$

$60 \quad \mathrm{POL}$

$65 \quad \mathrm{POL}$

$55 \quad \mathrm{POL}$

$45 \quad \mathrm{POL}$

$45 \quad$ POL

$55 \quad \mathrm{POL}$

$55 \quad \mathrm{POL}$

$50 \quad \mathrm{POL}$

$50 \quad \mathrm{POL}$

$55 \quad \mathrm{POL}$

$60 \quad \mathrm{POL}$

$40 \quad \mathrm{POL}$

$45 \quad \mathrm{POL}$

$50 \quad \mathrm{POL}$

$40 \quad \mathrm{POL}$

$40 \quad$ VIF

40

40

50

VIF

VIF

VIF

VIF

VIF

VIF

VIF

VIF

VIF

VIF

VIF

VIF

VIF

VIF

VIF

VIF

VIF

VIF

VIF

VIF

VIF

VIF

VIF

VIF

VIF 
TCTGTCAGTTTCGTAACACTAGG TAGTGTTACGAAACTGACAGAGG CGAAACTGACAGAGGATAGATGG GAACAAGCCCCAGAAGACCAAGG AACAAGCCCCAGAAGACCAAGGG CTGTGGCCCTTGGTCTTCTGGGG TCTGTGGCCCTTGGTCTTCTGGG CTCTGTGGCCCTTGGTCTTCTGG AGAAGACCAAGGGCCACAGAGGG GTGGCTCCCTCTGTGGCCCTTGG AGAGGGAGCCACACAATGAATGG TCTAGTGTCCATTCATTGTGTGG ATGGACACTAGAGCTTTTAGAGG TTTCCTAGGATTTGGCTCCATGG AAGCCATGGAGCCAAATCCTAGG AGGATTTGGCTCCATGGCTTAGG GGATTTGGCTCCATGGCTTAGGG AGATATGTTGCCCTAAGCCATGG ACTTATGGGGATACTTGGGCAGG TGGGGATACTTGGGCAGGAGTGG GTCGACACCCAATTCTGAAATGG GGGTGTCGACATAGCAGAATAGG GAATAGGCGTTACTCAACAGAGG CAACAGAGGAGAGCAAGAAATGG GGCTCTAGTCTAGGATCTACTGG TAGATCCTAGACTAGAGCCCTGG TGCTTCCAGGGCTCTAGTCTAGG CTAGAGCCCTGGAAGCATCCAGG TGACTTCCTGGATGCTTCCAGGG CTGACTTCCTGGATGCTTCCAGG GCAGTTTTAGGCTGACTTCCTGG CAAGTGGTACAAGCAGTTTTAGG AAAGCCTTAGGCATCTCCTATGG CCTTAGGCATCTCCTATGGCAGG TCTCCTATGGCAGGAAGAAGCGG TCTCCGCTTCTTCCTGCCATAGG TGAGTCTGACTGCCTTGAGGAGG TGATGAGTCTGACTGCCTTGAGG ATAGAAAGAGCAGAAGACAGTGG GACAGTGGCAATGAGAGTGAAGG AGGAGAAATATCAGCACTTGTGG AATATCAGCACTTGTGGAGATGG ATATCAGCACTTGTGGAGATGGG TATCAGCACTTGTGGAGATGGGG ATCAGCACTTGTGGAGATGGGGG AGCACTTGTGGAGATGGGGGTGG AAATGGGGCACCATGCTCCTTGG AATGGGGCACCATGCTCCTTGGG CATCAATATCCCAAGGAGCATGG GGGAGCAGCAGGAAGCACTATGG GGAGCAGCAGGAAGCACTATGGG CAGGAAGCACTATGGGCGCACGG CGCACGGTCAATGACGCTGACGG GTCAATGACGCTGACGGTACAGG CAGGCCAGACAATTATTGTCTGG GCAGCAGAACAATTTGCTGAGGG CAATTTGCTGAGGGCTATTGAGG ATCTGTTGCAACTCACAGTCTGG TCTGTTGCAACTCACAGTCTGGG CTGTTGCAACTCACAGTCTGGGG CTGGGGCATCAAGCAGCTCCAGG GCAGCTCCAGGCAAGAATCCTGG CCAGGCAAGAATCCTGGCTGTGG TTAGGTATCTTTCCACAGCCAGG GGCTGTGGAAAGATACCTAAAGG AGACCCACCTCCCAACCCCGAGG GACCCACCTCCCAACCCCGAGGG ACCCACCTCCCAACCCCGAGGGG TCCCCTCGGGGTTGGGAGGTGGG TGTCGGGTCCCCTCGGGGTTGGG TCTATTCCTTCGGGCCTGTCGGG sequence:5566

sequence: 5568

sequence: 5576

sequence: 5598

sequence:5599

sequence: 5605

sequence:5606

sequence: 5607

sequence:5609

sequence: 5615

sequence: 5626

sequence: 5634

sequence: 5645

sequence: 5694

sequence: 5697

sequence: 5700

sequence:5701

sequence:5711

sequence: 5739

sequence: 5744

sequence:5804

sequence:5817

sequence:5833

sequence: 5847

sequence:5872

sequence:5876

sequence:5881

sequence:5887

sequence:5893

sequence:5894

sequence:5905

sequence:5917

sequence:5986

sequence:5990

sequence:5999

sequence:6002

sequence: 6037

sequence:6040

sequence:6232

sequence: 6247

sequence:6267

sequence: 6273

sequence: 6274

sequence: 6275

sequence: 6276

sequence: 6279

sequence:6302

sequence:6303

sequence:6312

sequence:7840

sequence:7841

sequence:7848

sequence:7864

sequence:7870

sequence:7889

sequence:7921

sequence:7930

sequence:7962

sequence:7963

sequence:7964

sequence:7981

sequence:7993

sequence:7999

sequence:8011

sequence:8014

sequence:8424

sequence: 8425

sequence: 8426

sequence: 8427

sequence:8434

sequence: 8450
40

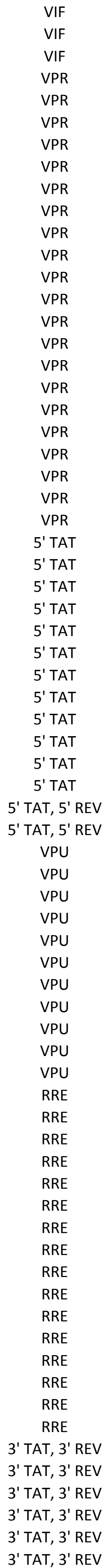


TTCTATTCCTTCGGGCCTGTCGG CCCGAAGGAATAGAAGAAGAAGG GGATCCGTTCACTAATCGAATGG ACGGATCCTTAGCACTTATCTGG CGGATCCTTAGCACTTATCTGGG ATCGTCCCAGATAAGTGCTAAGG CACTTATCTGGGACGATCTGCGG GGTGGTAGCTGAAGAGGCACAGG TCAAGCGGTGGTAGCTGAAGAGG AGAGTAAGTCTCTCAAGCGGTGG TCAAGAGTAAGTCTCTCAAGCGG CTTGATTGTAACGAGGATTGTGG AACGAGGATTGTGGAACTTCTGG ACGAGGATTGTGGAACTTCTGGG TTGTGGAACTTCTGGGACGCAGG TGTGGAACTTCTGGGACGCAGGG GTGGAACTTCTGGGACGCAGGGG TGGAACTTCTGGGACGCAGGGGG ACTTCTGGGACGCAGGGGGTGGG GGTGGGAAGCCCTCAAATATTGG GGGAAGCCCTCAAATATTGGTGG GGAGATTCCACCAATATTTGAGG TCTCCTACAGTATTGGAGTCAGG GTTCCTGACTCCAATACTGTAGG CAAGTGGTCAAAAAGTAGTGTGG TGGTCAAAAAGTAGTGTGGTTGG CAAAAAGTAGTGTGGTTGGATGG TGGTTGGATGGCCTACTGTAAGG GGTTGGATGGCCTACTGTAAGGG CTCATTCTTTCCCTTACAGTAGG CGAGCTGAGCCAGCAGCAGATGG GAGCTGAGCCAGCAGCAGATGGG AGCTGAGCCAGCAGCAGATGGGG TGAGCCAGCAGCAGATGGGGTGG GCTCCCACCCCATCTGCTGCTGG GGGAGCAGCATCTCGAGACCTGG TCTCGAGACCTGGAAAAACATGG TGATTGCTCCATGTTTTTCCAGG CTACCAATGCTGCTTGTGCCTGG TAGCCAGGCACAAGCAGCATTGG TGCCTGGCTAGAAGCACAAGAGG CTCCTCTTGTGCTTCTAGCCAGG CTGGCTAGAAGCACAAGAGGAGG GCTAGAAGCACAAGAGGAGGAGG GGGTTTTCCAGTCACACCTCAGG AAAGGTACCTGAGGTGTGACTGG ATTGGTCTTAAAGGTACCTGAGG AAAGAAAAGGGGGGACTGGAAGG AAGAAAAGGGGGGACTGGAAGGG sequence:8451 sequence: 8459 sequence: 8505 sequence: 8520 sequence: 8521 sequence: 8526 sequence: 8532 sequence: 8557 sequence: 8563 sequence: 8575 sequence: 8578 sequence: 8596 sequence:8605 sequence: 8606 sequence: 8613 sequence: 8614 sequence: 8615 sequence: 8616 sequence: 8620 sequence: 8637 sequence: 8640 sequence: 8647 sequence: 8665 sequence: 8668 sequence: 8852 sequence: 8856 sequence: 8860 sequence: 8872 sequence: 8873 sequence: 8883 sequence: 8907 sequence: 8908 sequence: 8909 sequence:8912 sequence:8916 sequence: 8933 sequence: 8943 sequence: 8951 sequence:8992 sequence:8995 sequence:9008 sequence:9010 sequence:9011 sequence:9014 sequence:9041 sequence:9048 sequence:9057 sequence:9117 sequence:9118
3' TAT, 3' REV

3' TAT, 3' REV 3' REV 3' REV

3' REV

3' REV

3' REV

3' REV

3' REV

3' REV

3' REV

3' REV

3' REV

3' REV

3' REV

3' REV

3' REV

3' REV

3' REV

3' REV

3' REV

3' REV

3' REV

3' REV

NEF

NEF

NEF

NEF

NEF

NEF

NEF

NEF

NEF

NEF

NEF

NEF

NEF

NEF

NEF

NEF

NEF

NEF

NEF

NEF

NEF

NEF

NEF

NEF

NEF 


\begin{tabular}{|c|c|c|c|c|}
\hline LAI Target sequence & 5 ' end of gRNA target site & Strand & $\% \mathrm{GC}$ content & HIV-1 target \\
\hline$\overline{\text { TACCACACACAAGGCTACTTCCCTGATT }}$ & sequence:54, 9186 & + & 45 & LTR U3 NEF \\
\hline СCTTATCTGGCTCAACTGGTACTAGCTT & sequence:141, 9273 & - & 50 & LTR U3 NEF \\
\hline AGGCCAATAAAGGAGAGAACACCAGCTT & sequence:175, 9307 & + & 45 & LTR U3 NEF \\
\hline TACTTCAAGAACTGCTGACATCGAGCTT & sequence: 315,9447 & + & 40 & LTR U3 NEF \\
\hline CTCAGATGCTGCATATAAGCAGCTGCTT & sequence: 414,9546 & + & 45 & LTR U3 \\
\hline ACCCAGTACAGGCAAAAAGCAGCTGCTT & sequence: 430,9562 & - & 50 & LTR U3 \\
\hline TACTGGGTCTCTCTGGTTAGACCAGATT & sequence:450, 9582 & + & 50 & LTR U3 \\
\hline СTCTGGCTAACTAGGGAACCCACTGCTT & sequence:492, 9624 & + & 55 & LTR R \\
\hline ACCCACTGCTTAAGCCTCAATAAAGCTT & sequence:509, 9641 & + & 50 & LTR R \\
\hline GCACTCAAGGCAAGCTTTATTGAGGCTT & sequence:520, 9652 & - & 45 & LTR R \\
\hline GCCTCAATAAAGCTTGCCTTGAGTGCTT & sequence:522, 9654 & + & 45 & LTR R \\
\hline CACTACTTGAAGCACTCAAGGCAAGCTT & sequence:531, 9663 & - & 45 & LTR R \\
\hline GAGAGCTCCTCTGGTTTCCCTTTCGCTT & sequence:658 & - & 60 & \\
\hline GGAGCTCTCTCGACGCAGGACTCGGCTT & sequence:677 & + & 65 & \\
\hline CTCCCCTCGCCTCTTGCCGTGCGCGCTT & sequence:709 & - & 70 & DIS \\
\hline TTCCCATCGATCTAATTCTCCCCCGCTT & sequence:813 & - & 40 & GAG \\
\hline TATGGGCAAGCAGGGAGCTAGAACGATT & sequence:893 & + & 55 & GAG \\
\hline AACTGCGAATCGTTCTAGCTCCCTGCTT & sequence:900 & - & 45 & GAG \\
\hline CTTCTGATGTTTCTAACAGGCCAGGATT & sequence:928 & - & 40 & GAG \\
\hline TGCTCTTCCTCTATCTTGTCTAAAGCTT & sequence:1085 & - & 45 & GAG \\
\hline CCTGTGTCAGCTGCTGCTTGCTGTGCTT & sequence:1130 & - & 60 & GAG \\
\hline CTGCTGTGTCCTGTGTCAGCTGCTGCTT & sequence:1139 & - & 60 & GAG \\
\hline TTATCAGAAGGAGCCACCCCACAAGATT & sequence:1312 & + & 55 & GAG \\
\hline GGATGCACTCTATCCCATTCTGCAGCTT & sequence:1412 & - & 50 & GAG \\
\hline TTGTTGGTCCAAAATGCGAACCCAGATT & sequence: 1750 & + & 40 & GAG \\
\hline TCTAGTGTAGCTGCTGGTCCCAATGCTT & sequence:1793 & - & 55 & GAG \\
\hline GCTGAATTTGTTACTTGGCTCATTGCTT & sequence:1883 & - & 40 & GAG \\
\hline GGAAAGGAAGGACACCAAATGAAAGATT & sequence:2038 & + & 45 & GAG \\
\hline AGCCAACAGCCCCACCAGAAGAGAGCTT & sequence: 2186 & + & 60 & GAG POL \\
\hline ACAGTTCCTTGTCTATCGGCTCCTGCTT & sequence: 2248 & - & 50 & GAG POL \\
\hline TCATCTGCTCCTGTATCTAATAGAGCTT & sequence: 2350 & - & 40 & POL \\
\hline GGTCCTACTAATACTGTACCTATAGCTT & sequence: 2497 & - & 45 & POL \\
\hline AAGTGCAACCAATCTGAGTCAACAGATT & sequence: 2550 & - & 45 & POL \\
\hline TAACTTTTGGGCCATCCATTCCTGGCTT & sequence: 2622 & - & 40 & POL \\
\hline TGGCAAATACTGGAGTATTGTATGGATT & sequence: 2745 & - & 40 & POL \\
\hline TCACCCACATCCAGTACTGTTACTGATT & sequence: 2896 & - & 50 & POL \\
\hline GGGAAAATTGAATTGGGCAAGTCAGATT & sequence:3368 & + & 40 & POL \\
\hline TTGGGCAAGTCAGATTTACCCAGGGATT & sequence: 3380 & + & 45 & POL \\
\hline CTGTTTTCTGCCAGTTCTAGCTCTGCTT & sequence:3478 & - & 45 & POL \\
\hline GCTAGAACTGGCAGAAAACAGAGAGATT & sequence:3485 & + & 45 & POL \\
\hline GATATGTCCATTGGCCTTGCCCCTGCTT & sequence:3576 & - & 50 & POL \\
\hline AGGAGTCTTTCCCCATATTACTATGCTT & sequence:3719 & - & 40 & POL \\
\hline GACAGAGTATTGGCAAGCCACCTGGATT & sequence:3791 & + & 50 & POL \\
\hline AACTCCCACTCAGGAATCCAGGTGGCTT & sequence:3805 & - & 50 & POL \\
\hline GAATTGCTTGTAACTCAGTCTTCTGATT & sequence:4005 & - & 40 & POL \\
\hline GAATCCTGCAAAGCTAGATGAATTGCTT & sequence: 4024 & - & 45 & POL \\
\hline TTCATCTAGCTTTGCAGGATTCGGGATT & sequence: 4030 & + & 40 & POL \\
\hline AGTAATTGGAGAGCAATGGCTAGTGATT & sequence: 4314 & + & 45 & POL \\
\hline CTACAGTCTACTTGTCCATGCATGGCTT & sequence: 4408 & - & 45 & POL \\
\hline TGTAGTCCAGGAATATGGCAACTAGATT & sequence:4431 & + & 45 & POL \\
\hline СCTGTTTCTGCTGGAATAACTTCTGCTT & sequence: 4519 & - & 45 & POL \\
\hline GTAGGGAATTCCAAATTCCTGCTTGATT & sequence:4667 & - & 40 & POL \\
\hline CTGCCCCTTCACCTTTCCAGAGGAGCTT & sequence: 4983 & - & 60 & POL \\
\hline TATGGAAAACAGATGGCAGGTGATGATT & sequence:5076 & + & 45 & POL \\
\hline TGTGTGGCAAGTAGACAGGATGAGGATT & sequence:5103 & + & 50 & $\mathrm{POL}$ \\
\hline TACACATCCCACTAGGGGATGCTAGATT & sequence: 5240 & + & 50 & VIF \\
\hline AGAGATCCTACCTTGTTATGTCCTGCTT & sequence: 5483 & - & 40 & VIF \\
\hline GTTTCGTAACACTAGGCAAAGGTGGCTT & sequence: 5554 & - & 40 & VIF \\
\hline CTCTGTGGCCCTTGGTCTTCTGGGGCTT & sequence:5602 & - & 60 & VPR \\
\hline GCCACACAATGAATGGACACTAGAGCTT & sequence:5633 & + & 50 & VPR \\
\hline ATGGACACTAGAGCTTTTAGAGGAGCTT & sequence:5645 & + & 40 & VPR \\
\hline ATTTTCCTAGGATTTGGCTCCATGGCTT & sequence:5692 & + & 40 & VPR \\
\hline CAGTTTTAGGCTGACTTCCTGGATGCTT & sequence:5899 & - & 45 & 5' TAT \\
\hline CATCCAGGAAGTCAGCCTAAAACTGCTT & sequence:5902 & + & 50 & $5^{\prime}$ TAT \\
\hline TTCCTGCCATAGGAGATGCCTAAGGCTT & sequence:5987 & - & 55 & 5' TAT \\
\hline GAGGAGGTCTTCGTCGCTGTCTCCGCTT & sequence:6016 & - & 60 & 5' TAT 5' REV \\
\hline CGTCATTGACCGTGCGCCCATAGTGCTT & sequence:7852 & - & 65 & RRE \\
\hline CAGCCAGGATTCTTGCCTGGAGCTGCTT & sequence:7991 & - & 60 & RRE \\
\hline CTTTAGGTATCTTTCCACAGCCAGGATT & sequence: 8008 & - & 40 & RRE \\
\hline AGAGAGACAGAGACAGATCCATTCGATT & sequence: 8487 & + & 50 & 3' REV \\
\hline GCCTGTGCCTCTTCAGCTACCACCGCTT & sequence:8556 & + & 60 & 3' REV \\
\hline CCACCGCTTGAGAGACTTACTCTTGATT & sequence:8575 & + & 55 & 3' REV \\
\hline GTTCCTGACTCCAATACTGTAGGAGATT & sequence:8663 & - & 45 & 3' REV \\
\hline
\end{tabular}


GGTAGCTGCTGTATTGCTACTTGTGATT AGCAATACAGCAGCTACCAATGCTGCTT AAACCCACCTCCTCCTCCTCTTGTGCTT sequence:8969

sequence: 8979

sequence:9019
NEF

NEF

NEF 


\begin{tabular}{|c|c|c|c|c|}
\hline LAI Target sequence & 5' end of gRNA target site & Strand & \% GC content & HIV-1 target \\
\hline GAGCGTCAGTATTAAGCGGGGGAGAAT & sequence: 800 & + & 55 & GAG \\
\hline ATTCGGTTAAGGCCAGGGGGAAAGAAA & sequence:844 & + & 60 & GAG \\
\hline TCGCAGTTAATCCTGGCCTGTTAGAAA & sequence:920 & + & 55 & GAG \\
\hline GAGGAAGAGCAAAACAAAAGTAAGAAA & sequence:1102 & + & 40 & GAG \\
\hline AGACCATCAATGAGGAAGCTGCAGAAT & sequence:1397 & + & 45 & GAG \\
\hline ATAATCCACCTATCCCAGTAGGAGAAA & sequence:1544 & + & 40 & GAG \\
\hline TGGGACCAGCAGCTACACTAGAAGAAA & sequence:1799 & + & 55 & GAG \\
\hline AAGAGGCAATTTTAGGAACCAAAGAAA & sequence:1926 & + & 40 & GAG \\
\hline TGGCAAAGAAGGGCACATAGCCAGAAA & sequence:1974 & + & 50 & GAG \\
\hline TGTTGGCTCTGGTCTGCTCTGAAGAAA & sequence:2131 & - & 55 & GAG POL \\
\hline TGTTGGCTCTGGTCTGCTCTGAAGAAA & sequence: 2167 & - & 55 & GAG POL \\
\hline GACAGTATGATCAGATACTCATAGAAA & sequence: 2458 & + & 40 & $\mathrm{POL}$ \\
\hline CCACATCCCGCAGGGTTAAAAAAGAAA & sequence:2868 & + & 55 & POL \\
\hline ACCACACCAGACAAAAAACATCAGAAA & sequence: 3228 & + & 40 & POL \\
\hline CAGTACAGCCTATAGTGCTGCCAGAAA & sequence:3304 & + & 50 & POL \\
\hline AAGAAGCAGAGCTAGAACTGGCAGAAA & sequence: 3475 & + & 45 & $\mathrm{POL}$ \\
\hline СACTCCATGTACTGGTTCTTTTAGAAT & sequence: 3510 & - & 45 & POL \\
\hline AGGCAGTGCAAAAAATAACCACAGAAA & sequence:3694 & + & 40 & POL \\
\hline AGAAAGAACCCATAGTAGGAGCAGAAA & sequence:3874 & + & 40 & POL \\
\hline GATAAGGCCCAAGATGAACATGAGAAA & sequence:4281 & + & 45 & POL \\
\hline ACCTGCCACCTGTAGTAGCAAAAGAAA & sequence:4345 & + & 55 & POL \\
\hline ATCCCCAAAGTCAAGGAGTAGTAGAAT & sequence:4696 & + & 45 & POL \\
\hline TGGGGGGTACAGTGCAGGGGAAAGAAT & sequence:4838 & + & 70 & POL cPPT \\
\hline TCACTATGAAAGCCCTCATCCAAGAAT & sequence:5202 & + & 45 & VIF \\
\hline CATATTGGGGTCTGCATACAGGAGAAA & sequence:5279 & + & 45 & VIF \\
\hline ATCTGGGTCAGGGAGTCTCCATAGAAT & sequence: 5315 & + & 60 & VIF \\
\hline CTGTTTTTCAGACTCTGCTATAAGAAA & sequence:5415 & + & 40 & VIF \\
\hline CTAGAGCTTTTAGAGGAGCTTAAGAAT & sequence:5652 & + & 45 & VPR \\
\hline GGCAGGAGTGGAAGCCATAATAAGAAT & sequence:5756 & + & 55 & VPR \\
\hline CAGAATTGGGTGTCGACATAGCAGAAT & sequence:5810 & + & 45 & VPR \\
\hline CGTTACTCAACAGAGGAGAGCAAGAAA & sequence:5840 & + & 50 & VPR \\
\hline TTGTGTGGTCCATAGTAATCATAGAAT & sequence: 6155 & + & 40 & VPU \\
\hline ACAGTGGCAATGAGAGTGAAGGAGAAA & sequence:6248 & + & 45 & VPU \\
\hline GGGCATCAAGCAGCTCCAGGCAAGAAT & sequence:7984 & + & 65 & RRE \\
\hline CAGTATTGGAGTCAGGAACTAAAGAAT & sequence:8672 & + & 45 & 3' REV \\
\hline TGGATGGCCTACTGTAAGGGAAAGAAT & sequence: 8876 & + & 55 & NEF \\
\hline
\end{tabular}




\begin{tabular}{|c|c|c|c|c|}
\hline LAI Target sequence & 5' end of gRNA target site & Strand & \% GC content & HIV-1 target \\
\hline ATATCTTGTCTTCGTTGGGAGTGAAT & sequence:12, 9144 & - & 40 & LTR U3 NEF \\
\hline GTGTGGTAGATCCACAGATCAAGGAT & sequence:36, 9168 & - & 50 & LTR U3 NEF \\
\hline CAGAACTACACACCAGGGCCAGGGGT & sequence:84, 9216 & + & 60 & LTR U3 NEF \\
\hline GGTCAGATATCCACTGACCTTTGGAT & sequence:107, 9239 & + & 50 & LTR U3 NEF \\
\hline GTAGCACCATCCAAAGGTCAGTGGAT & sequence:115, 9247 & - & 50 & LTR U3 NEF \\
\hline TTACACCCTGTGAGCCTGCATGGAAT & sequence:204, 9336 & + & 55 & LTR U3 NEF \\
\hline ACCCTGTGAGCCTGCATGGAATGGAT & sequence:208, 9340 & + & 60 & LTR U3 NEF \\
\hline TGACCCTGAGAGAGAAGTGTTAGAGT & sequence:233, 9365 & + & 50 & LTR U3 NEF \\
\hline ССАСТСТАAСАСТTСТСТСTCAGGGT & sequence:235, 9367 & - & 45 & LTR U3 NEF \\
\hline CGTGGCCCGAGAGCTGCATCCGGAGT & sequence:290, 9422 & + & 70 & LTR U3 NEF \\
\hline TCAGCAGTTCTTGAAGTACTCCGGAT & sequence:307, 9439 & - & 40 & LTR U3 NEF \\
\hline GCAGCTGCTTTTTTGCCTGTACTGGGT & sequence:432, 9564 & + & 50 & LTR U3 \\
\hline GCTTTATTGAGGCTTAAGCAGTGGGT & sequence:509, 9641 & - & 40 & LTR R \\
\hline AAGCCTCAATAAAGCTTGCCTTGAGT & sequence:520, 9652 & + & 45 & LTR R \\
\hline TCTGAGGGATCTCTAGTTACCAGAGT & sequence:578, 9710 & - & 45 & LTR U5 \\
\hline ACACTGACTAAAAGGGTCTGAGGGAT & sequence:594, 9726 & - & 45 & LTR U5 \\
\hline GCCGTGCGCGCTTCAGCAAGCCGAGT & sequence:696 & - & 70 & DIS \\
\hline CGGAGGCTAGAAGGAGAGAGATGGGT & sequence:770 & + & 60 & Psi \\
\hline AGCGTCAGTATTAAGCGGGGGAGAAT & sequence:801 & + & 55 & GAG \\
\hline TTCTTTCCCCCTGGCCTTAACCGAAT & sequence:844 & - & 50 & GAG \\
\hline CTAACAGGCCAGGATTAACTGCGAAT & sequence:918 & - & 45 & GAG \\
\hline TTCTGATGTTTCTAACAGGCCAGGAT & sequence:929 & - & 40 & GAG \\
\hline GCTACAACCATCCCTTCAGACAGGAT & sequence:978 & + & 50 & GAG \\
\hline GTTCTTCTGATCCTGTCTGAAGGGAT & sequence:987 & - & 45 & GAG \\
\hline АСССTCTATTGTGTGCATCAAAGGAT & sequence:1039 & + & 45 & GAG \\
\hline CCCCTGGATGTTCTGCACTATAGGGT & sequence:1184 & - & 55 & GAG \\
\hline GCCTGATGTACCATTTGCCCCTGGAT & sequence:1201 & - & 55 & GAG \\
\hline GACCATCAATGAGGAAGCTGCAGAAT & sequence:1398 & + & 50 & GAG \\
\hline TCAATGAGGAAGCTGCAGAATGGGAT & sequence:1403 & + & 45 & GAG \\
\hline GAGGAAGCTGCAGAATGGGATAGAGT & sequence:1408 & + & 55 & GAG \\
\hline TGCAATAGGCCCTGCATGCACTGGAT & sequence:1436 & - & 55 & GAG \\
\hline TAGTACCCTTCAGGAACAAATAGGAT & sequence:1509 & + & 40 & GAG \\
\hline ACCCTTCAGGAACAAATAGGATGGAT & sequence:1513 & + & 45 & GAG \\
\hline АATTTCTCCTACTGGGATAGGTGGAT & sequence:1547 & - & 40 & GAG \\
\hline TTTGGTCCTTGTCTTATGTCCAGAAT & sequence:1633 & - & 40 & GAG \\
\hline ACCGGTCTACATAGTCTCTAAAGGGT & sequence:1662 & - & 45 & GAG \\
\hline TGTGAAGCTTGCTCGGCTCTTAGAGT & sequence:1696 & - & 55 & GAG \\
\hline GCTTCACAGGAGGTAAAAAATTGGAT & sequence:1714 & + & 40 & GAG \\
\hline GAAATGATGACAGCATGTCAGGGAGT & sequence:1822 & + & 40 & GAG \\
\hline GGAGGACCCGGCCATAAGGCAAGAGT & sequence:1849 & + & 70 & GAG \\
\hline CCAAAACTCTTGCCTTATGGCCGGGT & sequence:1854 & - & 45 & GAG \\
\hline СTTCCTACAAGGGAAGGCCAGGGAAT & sequence:2105 & + & 55 & GAG POL \\
\hline CCAGAAGAGAGCTTCAGGTCTGGGGT & sequence: 2200 & + & 55 & GAG POL \\
\hline ATCGGCTCCTGCTTCTGAGAGGGAGT & sequence:2236 & - & 55 & GAG POL \\
\hline GAGTGATCTGAGGGAAGTTAAAGGAT & sequence:2276 & - & 45 & GAG POL \\
\hline GTGACGAGGGGTCGTTGCCAAAGAGT & sequence: 2298 & - & 65 & GAG POL \\
\hline ССССTATCTTTATTGTGACGAGGGGT & sequence:2312 & - & 45 & GAG POL \\
\hline GGCAAATACTGGAGTATTGTATGGAT & sequence: 2746 & - & 40 & POL \\
\hline AATTGAACTTCCCAGAAGTCTTGAGT & sequence:2835 & - & 40 & POL \\
\hline GACTTCTGGGAAGTTCAATTAGGAAT & sequence:2841 & + & 40 & POL \\
\hline ATTAGGAATACCACATCCCGCAGGGT & sequence:2858 & + & 45 & POL \\
\hline TCAGTACAATGTGCTTCCACAGGGAT & sequence:3017 & + & 45 & POL \\
\hline TGTGCTTCCACAGGGATGGAAAGGAT & sequence:3026 & + & 55 & POL \\
\hline CAGAAAGAACCTCCATTCCTTTGGAT & sequence:3249 & + & 45 & POL \\
\hline AAGAACCTCCATTCCTTTGGATGGGT & sequence:3253 & + & 45 & POL \\
\hline GGAGTTCATAACCCATCCAAAGGAAT & sequence:3263 & - & 45 & POL \\
\hline GTACTGTCCATTTATCAGGATGGAGT & sequence:3284 & - & 40 & POL \\
\hline AGGCTGTACTGTCCATTTATCAGGAT & sequence:3289 & - & 40 & POL \\
\hline TGGGCAAGTCAGATTTACCCAGGGAT & sequence:3381 & + & 50 & POL \\
\hline TTAGTGCTTTGGTTCCTCTAAGGAGT & sequence: 3428 & - & 40 & POL \\
\hline CAGGAAAATATGCAAGAACGAGGGGT & sequence:3637 & + & 40 & POL \\
\hline AACATGGGAAACATGGTGGACAGAGT & sequence: 3773 & + & 45 & $\mathrm{POL}$ \\
\hline ACAGAGTATTGGCAAGCCACCTGGAT & sequence:3792 & + & 50 & POL \\
\hline TTGGCAAGCCACCTGGATTCCTGAGT & sequence:3800 & + & 55 & POL \\
\hline AGCCACCTGGATTCCTGAGTGGGAGT & sequence:3806 & + & 55 & POL \\
\hline GTATTGACAAACTCCCACTCAGGAAT & sequence:3816 & - & 45 & POL \\
\hline AGAACGTTTCTGCTCCTACTATGGGT & sequence:3881 & - & 45 & POL \\
\hline CCCGAATCCTGCAAAGCTAGATGAAT & sequence:4029 & - & 55 & POL \\
\hline TCATCTAGCTTTGCAGGATTCGGGAT & sequence:4031 & + & 40 & POL \\
\hline TCACTTTTATCTGGTTGTGCTTGAAT & sequence:4101 & - & 40 & POL \\
\hline AAGGAAAAGGTCTATCTGGCATGGGT & sequence:4167 & + & 45 & POL \\
\hline GCATGGGTACCAGCACACAAAGGAAT & sequence:4185 & + & 55 & POL \\
\hline GGACAAGTAGACTGTAGTCCAGGAAT & sequence:4419 & + & 50 & POL \\
\hline
\end{tabular}


CTGGCTACATGAACTGCTACCAGGAT GGTAGCAGTTCATGTAGCCAGTGGAT GTTTCCTGCCCTGTTTCTGCTGGAAT AAGGCCGCCTGTTGGTGGGCGGGAAT TTGGTGGGCGGGAATCAAGCAGGAAT GCGGGAATCAAGCAGGAATTTGGAAT CCTTGACTTTGGGGATTGTAGGGAAT CCCTACAATCCCCAAAGTCAAGGAGT TCCCCAAAGTCAAGGAGTAGTAGAAT GTCAAGGAGTAGTAGAATCTATGAAT TAAAAGAAAAGGGGGGATTGGGGGGT GGGGGGTACAGTGCAGGGGAAAGAAT CTTTGCTGGTCCTTTCCAAAGTGGAT ATGATTGTGTGGCAAGTAGACAGGAT GTGTGGCAAGTAGACAGGATGAGGAT GTATGTTTCAGGGAAAGCTAGGGGAT CACTATGAAAGCCCTCATCCAAGAAT CAGAAGTACACATCCCACTAGGGGAT ACCAATCTAGCATCCCCTAGTGGGAT CAGGAGAAAGAGACTGGCATCTGGGT AGAGACTGGCATCTGGGTCAGGGAGT TCTGGGTCAGGGAGTCTCCATAGAAT ACATATAGTTAGCCCTAGGTGTGAAT TCAAGCAGGACATAACAAGGTAGGAT CTAGTGTTACGAAACTGACAGAGGAT GCCACAGAGGGAGCCACACAATGAAT TAGAGCTTTTAGAGGAGCTTAAGAAT GAAGCTGTTAGACATTTTCCTAGGAT ACTTATGGGGATACTTGGGCAGGAGT GCAGGAGTGGAAGCCATAATAAGAAT TGGATAAACAGCAGTTGTTGCAGAAT TGTCGACACCCAATTCTGAAATGGAT AGAATTGGGTGTCGACATAGCAGAAT CCATTTCTTGCTCTCCTCTGTTGAGT ATGCTTCCAGGGCTCTAGTCTAGGAT AGCAGTTTTAGGCTGACTTCCTGGAT TGTGTGGTCCATAGTAATCATAGAAT GAGCAGAAGACAGTGGCAATGAGAGT ATATCAGCACTTGTGGAGATGGGGGT AAATGGGGCACCATGCTCCTTGGGAT GCTGCTTGATGCCCCAGACTGTGAGT GGCATCAAGCAGCTCCAGGCAAGAAT TTTAGGTATCTTTCCACAGCCAGGAT TGGCTGTGGAAAGATACCTAAAGGAT CTTCTATTCCTTCGGGCCTGTCGGGT CAGATCCATTCGATTAGTGAACGGAT AGGATCCGTTCACTAATCGAATGGAT GCTAAGGATCCGTTCACTAATCGAAT GATCGTCCCAGATAAGTGCTAAGGAT CCACAATCCTCGTTACAATCAAGAGT TGTGGAACTTCTGGGACGCAGGGGGT GGGAAGCCCTCAAATATTGGTGGAAT GGTGGAATCTCCTACAGTATTGGAGT AGTATTGGAGTCAGGAACTAAAGAAT GTGGTCAAAAAGTAGTGTGGTTGGAT GGATGGCCTACTGTAAGGGAAAGAAT CGAGCTGAGCCAGCAGCAGATGGGGT sequence: 4482 sequence: 4487 sequence: 4530 sequence: 4644 sequence: 4655 sequence: 4662 sequence: 4686 sequence: 4689 sequence: 4697 sequence: 4705 sequence: 4820 sequence: 4839 sequence: 4960 sequence: 5098 sequence:5104 sequence: 5163 sequence: 5203 sequence: 5234 sequence: 5245 sequence: 5297 sequence: 5305 sequence: 5316 sequence: 5454 sequence: 5481 sequence: 5567 sequence: 5621 sequence: 5653 sequence: 5679 sequence:5739 sequence: 5757 sequence:5781 sequence:5802 sequence:5811 sequence: 5844 sequence: 5879 sequence:5903 sequence: 6156 sequence:6239 sequence: 6274 sequence:6302 sequence:7972 sequence:7985 sequence:8009 sequence: 8013 sequence: 8449 sequence: 8500 sequence: 8503 sequence: 8507 sequence: 8524 sequence: 8593 sequence: 8614 sequence: 8640 sequence: 8658 sequence: 8673 sequence: 8855 sequence: 8877 sequence: 8907
POL
POL

POL

POL

POL

POL

$\mathrm{POL}$

POL

POL

$\mathrm{POL}$

POL CPPT

POL CPPT

POL

POL

POL

VIF

VIF

VIF

VIF

VIF

VIF

VIF

VIF

VIF

VIF

VPR

VPR

VPR

VPR

VPR

VPR

VPR

VPR

VPR

5' TAT

5 ' TAT

VPU

VPU

VPU

VPU

RRE

RRE

RRE

RRE

3' TAT 3' REV

3' REV

3' REV

3' REV

3' REV

3' REV

3' REV

3' REV

3' REV

3' REV

NEF

NEF

NEF 


\begin{tabular}{|c|c|c|c|c|}
\hline LAI Target sequence & 5' end of gRNA target site & Strand & \% GC content & HIV-1 target \\
\hline TTTGGATGGTGCTACAAGCTAGTACCAG & sequence:126, 9258 & + & 50 & LTR U3 NEF \\
\hline TTTATTGGCCTCTTCTACCTTATCTGGC & sequence:158, 9290 & - & 50 & LTR U3 NEF \\
\hline TTTGACAGCCGCCTAGCATTTCATCACG & sequence:264, 9396 & + & 54 & LTR U3 NEF \\
\hline TTTCATCACGTGGCCCGAGAGCTGCATC & sequence:282, 9414 & + & 62 & LTR U3 NEF \\
\hline TTTTTGCCTGTACTGGGTCTCTCTGGTT & sequence:440, 9572 & + & 54 & LTR U3 \\
\hline TTTTGCCTGTACTGGGTCTCTCTGGTTA & sequence: 441,9573 & + & 54 & LTR U3 \\
\hline TTTGCCTGTACTGGGTCTCTCTGGTTAG & sequence: 442,9574 & + & 54 & LTR U3 \\
\hline TTTGAGCCTGGGAGCTCTCTGGCTAACT & sequence:476, 9608 & + & 58 & LTR R \\
\hline TTTATTGAGGCTTAAGCAGTGGGTTCCC & sequence: 505,9637 & - & 54 & LTR R \\
\hline TTTCCACACTGACTAAAAGGGTCTGAGG & sequence:597, 9729 & - & 50 & LTR U5 \\
\hline TTTTCCACACTGACTAAAAGGGTCTGAG & sequence:598, 9730 & - & 50 & LTR U5 \\
\hline TTTTAGTCAGTGTGGAAAATCTCTAGCA & sequence:607, 9739 & + & 42 & LTR U5 \\
\hline TTTAGTCAGTGTGGAAAATCTCTAGCAG & sequence:608 & + & 46 & LTR U5 \\
\hline TTTCAAGTCCCTGTTCGGGCGCCACTGC & sequence:632 & - & 67 & LTR U5 \\
\hline TTTCGCTTTCAAGTCCCTGTTCGGGCGC & sequence:638 & - & 62 & PBS \\
\hline TTTCCCTTTCGCTTTCAAGTCCCTGTTC & sequence:644 & - & 50 & PBS \\
\hline TTTGGCGTACTCACCAGTCGCCTCCCCT & sequence:730 & - & 67 & DIS \\
\hline TTTTTGGCGTACTCACCAGTCGCCTCCC & sequence:732 & - & 67 & DIS \\
\hline TTTTGACTAGCGGAGGCTAGAAGGAGAG & sequence:760 & + & 58 & Psi \\
\hline TTTGACTAGCGGAGGCTAGAAGGAGAGA & sequence:761 & + & 54 & Psi \\
\hline TTTCCCATCGATCTAATTCTCCCCCGCT & sequence:814 & - & 54 & GAG \\
\hline TTTTCCCATCGATCTAATTCTCCCCCGC & sequence:815 & - & 58 & GAG \\
\hline TTTTTCCCATCGATCTAATTCTCCCCCG & sequence: 816 & - & 54 & GAG \\
\hline TTTTTTCCCATCGATCTAATTCTCCCCC & sequence:817 & - & 50 & GAG \\
\hline TTTCCCCCTGGCCTTAACCGAATTTTTT & sequence:839 & - & 46 & GAG \\
\hline TTTCTTTCCCCCTGGCCTTAACCGAATT & sequence: 843 & - & 50 & GAG \\
\hline ТTTTCTTTTCCCCCTGGCCTTAACCGAAT & sequence:844 & - & 54 & GAG \\
\hline TTTTTCTTTCCCCCTGGCCTTAACCGAA & sequence:845 & - & 54 & GAG \\
\hline TTTTTTCTTTCCCCCTGGCCTTAACCGA & sequence:846 & - & 54 & GAG \\
\hline TTTATATTTTTTCTTTCCCCCTGGCCTT & sequence:852 & - & 42 & GAG \\
\hline TTTCTAACAGGCCAGGATTAACTGCGAA & sequence:919 & - & 46 & GAG \\
\hline TTTGATGCACACAATAGAGGGTTGCTAC & sequence:1033 & - & 46 & GAG \\
\hline TTTGCTCTTCCTCTATCTTGTCTAAAGC & sequence:1087 & - & 42 & GAG \\
\hline TTTTGCTCTTCCTCTATCTTGTCTAAAG & sequence:1088 & - & 42 & GAG \\
\hline TTTAGACAAGATAGAGGAAGAGCAAAAC & sequence:1089 & + & 42 & GAG \\
\hline TTTTGTTTTGCTCTTCCTCTATCTTGTC & sequence:1093 & - & 42 & GAG \\
\hline TTTGGCTGACCTGGCTGCTGTGTCCTGT & sequence:1153 & - & 62 & GAG \\
\hline TTTTGGCTGACCTGGCTGCTGTGTCCTG & sequence:1154 & - & 67 & GAG \\
\hline TTTGCCCCTGGATGTTCTGCACTATAGG & sequence:1186 & - & 54 & GAG \\
\hline TTTAAAGTTCTAGGTGATATGGCCTGAT & sequence:1220 & - & 42 & GAG \\
\hline TTTACCCATGCATTTAAAGTTCTAGGTG & sequence:1232 & - & 42 & GAG \\
\hline TTTCAGCCCAGAAGTGATACCCATGTTT & sequence:1278 & + & 46 & GAG \\
\hline TTTTCAGCATTATCAGAAGGAGCCACCC & sequence:1303 & + & 54 & GAG \\
\hline TTTCAGCATTATCAGAAGGAGCCACCCC & sequence:1304 & + & 54 & GAG \\
\hline TTTAAATCTTGTGGGGTGGCTCCTTCTG & sequence:1316 & - & 54 & GAG \\
\hline TTTAGCATGGTGTTTAAATCTTGTGGGG & sequence:1328 & - & 46 & GAG \\
\hline TTTAAACACCATGCTAAACACAGTGGGG & sequence:1338 & + & 50 & GAG \\
\hline TTTGCATGGCTGCTTGATGTCCCCCCAC & sequence:1360 & - & 62 & GAG \\
\hline TTTAACATTTGCATGGCTGCTTGATGTC & sequence:1367 & - & 46 & GAG \\
\hline TTTTAACATTTGCATGGCTGCTTGATGT & sequence:1368 & - & 42 & GAG \\
\hline TTTGTTCCTGAAGGGTACTAGTAGTTCC & sequence:1501 & - & 46 & GAG \\
\hline TTTCTCCTACTGGGATAGGTGGATTATT & sequence:1543 & - & 42 & GAG \\
\hline TTTGGTCCTTGTCTTATGTCCAGAATGC & sequence:1631 & - & 46 & GAG \\
\hline TTTTGGTCCTTGTCTTATGTCCAGAATG & sequence:1632 & - & 46 & GAG \\
\hline TTTAGAGACTATGTAGACCGGTTCTATA & sequence:1666 & + & 42 & GAG \\
\hline TTTATAGAACCGGTCTACATAGTCTCTA & sequence:1668 & - & 42 & GAG \\
\hline TTTTATAGAACCGGTCTACATAGTCTCT & sequence:1669 & - & 42 & GAG \\
\hline TTTACCTCCTGTGAAGCTTGCTCGGCTC & sequence:1703 & - & 62 & GAG \\
\hline TTTTACCTCCTGTGAAGCTTGCTCGGCT & sequence:1704 & - & 58 & GAG \\
\hline TTTTTACCTCCTGTGAAGCTTGCTCGGC & sequence:1705 & - & 58 & GAG \\
\hline TTTTTTACCTCCTGTGAAGCTTGCTCGG & sequence:1706 & - & 54 & GAG \\
\hline TTTGGACCAACAAGGTTTCTGTCATCCA & sequence:1735 & - & 46 & GAG \\
\hline TTTTGGACCAACAAGGTTTCTGTCATCC & sequence:1736 & - & 50 & GAG \\
\hline TTTTAAAAGCATTGGGACCAGCAGCTAC & sequence:1787 & + & 50 & GAG \\
\hline TTTAAAAGCATTGGGACCAGCAGCTACA & sequence:1788 & + & 50 & GAG \\
\hline TTTCTTCTAGTGTAGCTGCTGGTCCCAA & sequence:1798 & - & 50 & GAG \\
\hline TTTTGGCTGAAGCAATGAGCCAAGTAAC & sequence:1874 & + & 50 & GAG \\
\hline TTTGGCTGAAGCAATGAGCCAAGTAACA & sequence:1875 & + & 46 & GAG \\
\hline TTTGTTACTTGGCTCATTGCTTCAGCCA & sequence:1877 & - & 46 & GAG \\
\hline TTTCAATTGTGGCAAAGAAGGGCACATA & sequence:1965 & + & 42 & GAG \\
\hline
\end{tabular}


TTTCTGGCTATGTGCCCTTCTTTGCCAC TTTCCTAGGGGCCCTGCAATTTCTGGCT TTTTCCTAGGGGCCCTGCAATTTCTGGC TTTTTCCTAGGGGCCCTGCAATTTCTGG TTTCCAACAGCCCTTTTTCCTAGGGGCC TTTCCACATTTCCAACAGCCCTTTTTCC TTTGGTGTCCTTCCTTTCCACATTTCCA TTTTTTAGGGAAGATCTGGCCTTCCTAC TTTTTAGGGAAGATCTGGCCTTCCTACA TTTTAGGGAAGATCTGGCCTTCCTACAA TTTAGGGAAGATCTGGCCTTCCTACAAG TTTTCTTCAGAGCAGACCAGAGCCAACA TTTCTTCAGAGCAGACCAGAGCCAACAG TTTCTTCAGAGCAGACCAGAGCCAACAG TTTAACTTCCCTCAGATCACTCTTTGGC TTTGGCAACGACCCCTCGTCACAATAAA TTTAGTTGCCCCCCTATCTTTATTGTGA TTTCCATCTTCCTGGCAAACTCATTTCT TTTGGTTTCCATCTTCCTGGCAAACTCA TTTTGGTTTCCATCTTCCTGGCAAACTC TTTTTGGTTTCCATCTTCCTGGCAAACT TTTGCCAGGAAGATGGAAACCAAAAATG TTTGATAAAACCTCCAATTCCCCCTATC TTTAAAGTGCAACCAATCTGAGTCAACA TTTGGGCCATCCATTCCTGGCTTTAATT TTTTGGGCCATCCATTCCTGGCTTTAAT TTTAACTTTTGGGCCATCCATTCCTGGC TTTTCCCTTCCTTTTCCATTTCTGTACA TTTAACCCTGCGGGATGTGGTATTCCTA TTTTAACCCTGCGGGATGTGGTATTCCT TTTTTAACCCTGCGGGATGTGGTATTCC TTTTTTAACCCTGCGGGATGTGGTATTC TTTCTTTTTTAACCCTGCGGGATGTGGT TTTTCTTTTTTAACCCTGCGGGATGTGG TTTTTCTTTTTTAACCCTGCGGGATGTG TTTTTCAGTTCCCTTAGATGAAGACTTC TTTTCAGTTCCCTTAGATGAAGACTTCA TTTCAGTTCCCTTAGATGAAGACTTCAG TTTCCATCCCTGTGGAAGCACATTGTAC TTTGGAATATTGCTGGTGATCCTTTCCA TTTTGTTCTATGCTGCCCTATTTCTAAG TTTGTCTGGTGTGGTAAGTCCCCACCTC TTTTGTCTGGTGTGGTAAGTCCCCACCT TTTTTGTCTGGTGTGGTAAGTCCCCACC TTTTTTGTCTGGTGTGGTAAGTCCCCAC TTTGGATGGGTTATGAACTCCATCCTGA TTTATCAGGATGGAGTTCATAACCCATC TTTCTGGCAGCACTATAGGCTGTACTGT TTTTCTGGCAGCACTATAGGCTGTACTG TTTTTCTGGCAGCACTATAGGCTGTACT TTTTCCCACTAACTTCTGTATGTCATTG TTTAATCCCTGGGTAAATCTGACTTGCC TTTACCCAGGGATTAAAGTAAGGCAATT TTTCTGCCAGTTCTAGCTCTGCTTCTTC TTTTCTGCCAGTTCTAGCTCTGCTTCTT TTTAGAATCTCTCTGTTTTCTGCCAGTT TTTTAGAATCTCTCTGTTTTCTGCCAGT TTTTGATGGGTCATAATACACTCCATGT TTTGATATGTCCATTGGCCTTGCCCCTG TTTACATCATTAGTGTGGGCACCCCTCG TTTTACATCATTAGTGTGGGCACCCCTC TTTCTGTGGTTATTTTTTGCACTGCCTC TTTCCCCATATTACTATGCTTTCTGTGG TTTAGGAGTCTTTCCCCATATTACTATG TTTCCCATGTTTCCTTTTGTATGGGTAG TTTCACTAAAGGAGGGGTATTGACAAAC TTTCTGCTCCTACTATGGGTTCTTTCTC TTTAGTCTCCCTGCTAGCTGCCCCATCT TTTCCTAATTTAGTCTCCCTGCTAGCTG TTTTCCTAATTTAGTCTCCCTGCTAGCT TTTGTTGTGTCAGTTAGGGTGACAACTT sequence:1973 sequence:1992 sequence:1993 sequence:1994 sequence: 2007 sequence: 2015 sequence:2029 sequence: 2085 sequence: 2086 sequence: 2087 sequence: 2088 sequence: 2130 sequence: 2131 sequence: 2167 sequence: 2280 sequence: 2302 sequence: 2320 sequence: 2390 sequence: 2395 sequence: 2396 sequence: 2397 sequence:2399 sequence: 2426 sequence: 2554 sequence: 2617 sequence: 2618 sequence: 2624 sequence: 2697 sequence:2860 sequence: 2861 sequence: 2862 sequence: 2863 sequence: 2867 sequence: 2868 sequence: 2869 sequence: 2930 sequence: 2931 sequence: 2932 sequence: 3020 sequence: 3042 sequence:3161 sequence:3215 sequence:3216 sequence:3217 sequence: 3218 sequence: 3268 sequence:3272 sequence:3303 sequence:3304 sequence:3305 sequence:3347 sequence: 3383 sequence: 3394 sequence: 3474 sequence: 3475 sequence: 3490 sequence: 3491 sequence: 3527 sequence: 3579 sequence: 3655 sequence: 3656 sequence:3693 sequence: 3712 sequence: 3722 sequence: 3756 sequence: 3830 sequence: 3873 sequence:3911 sequence:3919 sequence: 3920 sequence:3979
GAG

GAG

GAG

GAG

GAG

GAG

GAG

GAG POL

GAG POL

GAG POL

GAG POL

GAG POL

GAG POL

GAG POL

GAG POL

GAG POL

GAG POL

POL

POL

POL

POL

POL

POL

POL

POL

POL

POL

POL

POL

POL

POL

POL

POL

POL

POL

POL

POL

POL

POL

POL

POL

POL

POL

POL

POL

POL

POL

POL

POL

POL

POL

POL

POL

POL

POL

POL

POL

POL

POL

POL

POL

POL

POL

POL

POL

POL

POL

POL

POL

POL

POL 
TTTACTTCTAATCCCGAATCCTGCAAAG TTTATCTGGTTGTGCTTGAATGATTCCT TTTTATCTGGTTGTGCTTGAATGATTCC TTTGTGTGCTGGTACCCATGCCAGATAG TTTCCTCCAATTCCTTTGTGTGCTGGTA TTTTTAGATGGAATAGATAAGGCCCAAG TTTTAGATGGAATAGATAAGGCCCAAGA TTTAGATGGAATAGATAAGGCCCAAGAT TTTCTCATGTTCATCTTGGGCCTTATCT TTTGCTACTACAGGTGGCAGGTTAAAAT TTTTAACCTGCCACCTGTAGTAGCAAAA TTTAACCTGCCACCTGTAGTAGCAAAAG TTTCTTTTGCTACTACAGGTGGCAGGTT TTTAGCTGACATTTATCACAGCTGGCTA TTTTAGCTGACATTTATCACAGCTGGCT TTTAGAAGGAAAAGTTATCCTGGTAGCA TTTCCTGCCCTGTTTCTGCTGGAATAAC TTTAAAAGAAAGTATGCTGTTTCCTGCC TTTTAAAATTAGCAGGAAGATGGCCAGT TTTAAAATTAGCAGGAAGATGGCCAGTA TTTACTGGCCATCTTCCTGCTAATTTTA TTTTACTGGCCATCTTCCTGCTAATTTT TTTTTACTGGCCATCTTCCTGCTAATTT TTTCACCAGTACTACGGTTAAGGCCGCC TTTGGGGATTGTAGGGAATTCCAAATTC TTTGGAATTCCCTACAATCCCCAAAGTC TTTGTACTGCTGTCTTAAGATGTTCAGC TTTTAAAAGAAAAGGGGGGATTGGGGGG TTTAAAAGAAAAGGGGGGATTGGGGGGT TTTCCCCTGCACTGTACCCCCCAATCCC TTTTCGGGTTTATTACAGGGACAGCAGA TTTCGGGTTTATTACAGGGACAGCAGAG TTTATTACAGGGACAGCAGAGATCCACT TTTCCAAAGTGGATCTCTGCTGTCCCTG TTTGCTGGTCCTTTCCAAAGTGGATCTC TTTGGAAAGGACCAGCAAAGCTCCTCTG TTTCCAGAGGAGCTTTGCTGGTCCTTTC TTTCCATGTTCTAATCCTCATCCTGTCT TTTTCCATGTTCTAATCCTCATCCTGTC TTTCAGGGAAAGCTAGGGGATGGTTTTA TTTTATAGACATCACTATGAAAGCCCTC TTTATAGACATCACTATGAAAGCCCTCA TTTCTCCTGTATGCAGACCCCAATATGT TTTCCTCCATTCTATGGAGACTCCCTGA TTTTCCTCCATTCTATGGAGACTCCCTG TTTTTCCTCCATTCTATGGAGACTCCCT TTTTTCAGACTCTGCTATAAGAAAGGCC TTTTCAGACTCTGCTATAAGAAAGGCCT TTTCAGACTCTGCTATAAGAAAGGCCTT TTTGGTGTTATTAATGCTGCTAGTGCCA TTTTGGTGTTATTAATGCTGCTAGTGCC TTTTTGGTGTTATTAATGCTGCTAGTGC TTTCGTAACACTAGGCAAAGGTGGCTTT TTTGCCTAGTGTTACGAAACTGACAGAG TTTTAGAGGAGCTTAAGAATGAAGCTGT TTTAGAGGAGCTTAAGAATGAAGCTGTT TTTTCCTAGGATTTGGCTCCATGGCTTA TTTCCTAGGATTTGGCTCCATGGCTTAG TTTGGCTCCATGGCTTAGGGCAACATAT TTTCATAGATATGTTGCCCTAAGCCATG TTTATCCATTTCAGAATTGGGTGTCGAC TTTCAGAATTGGGTGTCGACATAGCAGA TTTCTTGCTCTCCTCTGTTGAGTAACGC TTTAGGCTGACTTCCTGGATGCTTCCAG TTTTAGGCTGACTTCCTGGATGCTTCCA TTTACAATAGCAAGTGGTACAAGCAGTT TTTTACAATAGCAAGTGGTACAAGCAGT TTTTTACAATAGCAAGTGGTACAAGCAG TTTCACAACAAAAGCCTTAGGCATCTCC TTTCTCCTTCACTCTCATTGCCACTGTC TTTCCACCCCCATCTCCACAAGTGCTGA sequence: 4039 sequence: 4094 sequence: 4095 sequence: 4178 sequence: 4192 sequence: 4266 sequence: 4267 sequence: 4268 sequence: 4280 sequence: 4339 sequence: 4340 sequence: 4341 sequence: 4344 sequence: 4375 sequence: 4376 sequence: 4466 sequence: 4527 sequence: 4546 sequence: 4567 sequence: 4568 sequence: 4570 sequence: 4571 sequence: 4572 sequence: 4625 sequence:4677 sequence: 4680 sequence: 4770 sequence: 4817 sequence:4818 sequence:4833 sequence:4931 sequence:4932 sequence:4939 sequence: 4946 sequence: 4957 sequence: 4966 sequence: 4970 sequence:5115 sequence:5116 sequence:5168 sequence:5191 sequence:5192 sequence:5278 sequence:5322 sequence:5323 sequence:5324 sequence:5418 sequence:5419 sequence: 5420 sequence:5519 sequence: 5520 sequence:5521 sequence:5553 sequence:5562 sequence:5659 sequence: 5660 sequence:5693 sequence:5694 sequence:5704 sequence: 5712 sequence:5799 sequence:5807 sequence:5839 sequence:5895 sequence: 5896 sequence: 5922 sequence:5923 sequence:5924 sequence:5976 sequence: 6247 sequence:6277
POL

POL

POL

POL

POL

POL

POL

POL

POL

POL

POL

POL

POL

$\mathrm{POL}$

POL

POL

$\mathrm{POL}$

POL

POL

POL

$\mathrm{POL}$

POL

POL

POL

POL

$\mathrm{POL}$

POL

POL CPPT

POL CPPT

POL cPPT

POL

POL

POL

POL

POL

$\mathrm{POL}$

POL

POL

$\mathrm{POL}$

VIF

VIF

VIF

VIF

VIF

VIF

VIF

VIF

VIF

VIF

VIF

VIF

VIF

VIF

VIF

VPR

VPR

VPR

VPR

VPR

VPR

VPR

VPR

VPR

5' TAT

5' TAT

5' TAT

5' TAT

5 ' TAT

5' TAT

VPU

VPU 
NL4-3

\begin{tabular}{|c|c|c|c|c|}
\hline NL4-3 Target sequence & 5' end of gRNA target site & Strand & \% GC content & HIV-1 target \\
\hline GACAAGAGATCCTTGATCTGTGG & sequence:28 & + & 45 & LTR U3 \\
\hline TGTGGTAGATCCACAGATCAAGG & sequence:38, 9113 & - & 45 & LTR U3 Nef \\
\hline CTGTGGATCTACCACACACAAGG & sequence: 45,9120 & + & 50 & LTR U3 Nef \\
\hline CAGGGAAGTAGCCTTGTGTGTGG & sequence:56, 9131 & - & 55 & LTR U3 Nef \\
\hline CACAAGGCTACTTCCCTGATTGG & sequence:61, 9136 & + & 50 & LTR U3 Nef \\
\hline GTGTGTAGTTCTGCCAATCAGGG & sequence: 74,9149 & - & 45 & LTR U3 Nef \\
\hline GGTGTGTAGTTCTGCCAATCAGG & sequence: 75,9150 & - & 50 & LTR U3 Nef \\
\hline GATTGGCAGAACTACACACCAGG & sequence:78, 9153 & + & 50 & LTR U3 Nef \\
\hline ATTGGCAGAACTACACACCAGGG & sequence:79, 9154 & + & 45 & LTR U3 Nef \\
\hline CAGAACTACACACCAGGGCCAGG & sequence:84, 9159 & + & 60 & LTR U3 Nef \\
\hline AGAACTACACACCAGGGCCAGGG & sequence: 85,9160 & + & 55 & LTR U3 Nef \\
\hline GGATATCTGATCCCTGGCCCTGG & sequence:96 & - & 60 & LTR U3 \\
\hline GTCAGTGGATATCTGATCCCTGG & sequence:102 & - & 50 & LTR U3 \\
\hline ATCAGATATCCACTGACCTTTGG & sequence:108 & + & 40 & LTR U3 \\
\hline GATATCCACTGACCTTTGGATGG & sequence:112, 9187 & + & 45 & LTR U3 Nef \\
\hline AAGCACCATCCAAAGGTCAGTGG & sequence:117 & - & 50 & LTR U3 \\
\hline TAACTTGAAGCACCATCCAAAGG & sequence:124 & - & 40 & LTR U3 \\
\hline TCTACTTGCTCTGGTTCAACTGG & sequence:150 & - & 45 & LTR U3 \\
\hline TGAACCAGAGCAAGTAGAAGAGG & sequence:155 & + & 45 & LTR U3 \\
\hline TTGGCCTCTTCTACTTGCTCTGG & sequence:159 & - & 50 & LTR U3 \\
\hline CAAGTAGAAGAGGCCAATGAAGG & sequence:165 & + & 45 & LTR U3 \\
\hline GCTGTTGTTCTCTCCTTCATTGG & sequence:178 & - & 45 & LTR U3 \\
\hline TTACACCCTATGAGCCAGCATGG & sequence:204 & + & 50 & LTR U3 \\
\hline TACACCCTATGAGCCAGCATGGG & sequence:205 & + & 50 & LTR U3 \\
\hline CCCTATGAGCCAGCATGGGATGG & sequence:209 & + & 60 & LTR U3 \\
\hline TCCATCCCATGCTGGCTCATAGG & sequence:210 & - & 55 & LTR U3 \\
\hline TATGAGCCAGCATGGGATGGAGG & sequence:212 & + & 55 & LTR U3 \\
\hline CCAGCATGGGATGGAGGACCCGG & sequence:218 & + & 65 & LTR U3 \\
\hline GCATGGGATGGAGGACCCGGAGG & sequence:221 & + & 70 & LTR U3 \\
\hline CATGGGATGGAGGACCCGGAGGG & sequence:222 & + & 65 & LTR U3 \\
\hline АСАCTAATACTTCTCCCTCCGGG & sequence:236 & - & 45 & LTR U3 \\
\hline CACACTAATACTTCTCCCTCCGG & sequence:237 & - & 45 & LTR U3 \\
\hline CGGAGGGAGAAGTATTAGTGTGG & sequence:238 & + & 50 & LTR U3 \\
\hline CCTCCTAGCATTTCGTCACATGG & sequence: 272 & + & 50 & LTR U3 \\
\hline GGGCCATGTGACGAAATGCTAGG & sequence: 275 & - & 55 & LTR U3 \\
\hline CATGGCCCGAGAGCTGCATCCGG & sequence:290 & + & 65 & LTR U3 \\
\hline GTACTCCGGATGCAGCTCTCGGG & sequence:295, 9370 & - & 60 & LTR U3 Nef \\
\hline AGTACTCCGGATGCAGCTCTCGG & sequence:296, 9371 & - & 55 & LTR U3 Nef \\
\hline CAGCAGTCTTTGTAGTACTCCGG & sequence:309 & - & 45 & LTR U3 \\
\hline CTGACATCGAGCTTTCTACAAGG & sequence:329 & + & 45 & LTR U3 \\
\hline TGACATCGAGCTTTCTACAAGGG & sequence:330 & + & 40 & LTR U3 \\
\hline TTCTACAAGGGACTTTCCGCTGG & sequence:342 & + & 50 & LTR U3 \\
\hline TCTACAAGGGACTTTCCGCTGGG & sequence:343 & + & 50 & LTR U3 \\
\hline CTACAAGGGACTTTCCGCTGGGG & sequence: 344,9419 & + & 55 & LTR U3 \\
\hline CTTTCCGCTGGGGACTTTCCAGG & sequence:354, 9429 & + & 60 & LTR U3 \\
\hline TTTCCGCTGGGGACTTTCCAGGG & sequence: 355,9430 & + & 55 & LTR U3 \\
\hline CCGCTGGGGACTTTCCAGGGAGG & sequence:358, 9433 & + & 70 & LTR U3 \\
\hline GGGGACTTTCCAGGGAGGTGTGG & sequence:363 & + & 65 & LTR U3 \\
\hline TTTCCAGGGAGGTGTGGCCTGGG & sequence:369 & + & 60 & LTR U3 \\
\hline CAGCTGCTTTTTGCCTGTACTGG & sequence: 433,9508 & + & 50 & LTR U3 \\
\hline AGCTGCTTTTTGCCTGTACTGGG & sequence:434, 9509 & + & 45 & LTR U3 \\
\hline TTGCCTGTACTGGGTCTCTCTGG & sequence:443, 9518 & + & 55 & LTR U3 \\
\hline TAACCAGAGAGACCCAGTACAGG & sequence:446, 9521 & - & 50 & LTR U3 \\
\hline GGTTAGACCAGATCTGAGCCTGG & sequence:464,9539 & + & 55 & LTR R \\
\hline GTTAGACCAGATCTGAGCCTGGG & sequence: 465,9540 & + & 50 & LTR R \\
\hline AGAGCTCCCAGGCTCAGATCTGG & sequence: 471,9546 & - & 60 & LTR R \\
\hline ATCTGAGCCTGGGAGCTCTCTGG & sequence: 475,9550 & + & 60 & LTR R \\
\hline TAGTTAGCCAGAGAGCTCCCAGG & sequence: 482,9557 & - & 55 & LTR R \\
\hline TGGGAGCTCTCTGGCTAACTAGG & sequence:484, 9559 & + & 55 & LTR R \\
\hline GGGAGCTCTCTGGCTAACTAGGG & sequence: 485,9560 & + & 55 & LTR R \\
\hline CTTTATTGAGGCTTAAGCAGTGG & sequence: 511,9586 & - & 40 & LTR R \\
\hline ACTCAAGGCAAGCTTTATTGAGG & sequence:523,9598 & - & 40 & LTR R \\
\hline CACACTACTTTGAGCACTCAAGG & sequence:538 & - & 45 & LTR R \\
\hline GCCCGTCTGTTGTGTGACTCTGG & sequence:562, 9637 & + & 60 & LTR U5 \\
\hline ACCAGAGTCACACAACAGACGGG & sequence:563, 9638 & - & 50 & LTR U5 \\
\hline TACCAGAGTCACACAACAGACGG & sequence:564, 9639 & - & 45 & LTR U5 \\
\hline CACTGACTAAAAGGGTCTGAGGG & sequence:596, 9671 & - & 45 & LTR U5 \\
\hline ACACTGACTAAAAGGGTCTGAGG & sequence:597, 9672 & - & 45 & LTR U5 \\
\hline TCAGACCCTTTTAGTCAGTGTGG & sequence:599, 9674 & + & 45 & LTR U5 \\
\hline GTGTGGAAAATCTCTAGCAGTGG & sequence:616 & + & 45 & LTR U5 \\
\hline
\end{tabular}


TCTAGCAGTGGCGCCCGAACAGG CTAGCAGTGGCGCCCGAACAGGG TCGCTTTCAAGTCCCTGTTCGGG TTCGCTTTCAAGTCCCTGTTCGG GAAAGCGAAAGTAAAGCCAGAGG CTGCGTCGAGAGATCTCCTCTGG CAGAGGAGATCTCTCGACGCAGG AGATCTCTCGACGCAGGACTCGG TCGGCTTGCTGAAGCGCGCACGG GCTGAAGCGCGCACGGCAAGAGG CCAAAAATTTTGACTAGCGGAGG TTTGACTAGCGGAGGCTAGAAGG GAGGCTAGAAGGAGAGAGATGGG AGAGATGGGTGCGAGAGCGTCGG GCGAGAGCGTCGGTATTAAGCGG CGAGAGCGTCGGTATTAAGCGGG GAGAGCGTCGGTATTAAGCGGGG AGAGCGTCGGTATTAAGCGGGGG GCGGGGGAGAATTAGATAAATGG CGGGGGAGAATTAGATAAATGGG GAAAAAATTCGGTTAAGGCCAGG AAAATTCGGTTAAGGCCAGGGGG AACATATAGTATGGGCAAGCAGG ACATATAGTATGGGCAAGCAGGG GAACGATTCGCAGTTAATCCTGG GGCCTTTTAGAGACATCAGAAGG AGCCTTCTGATGTCTCTAAAAGG AGAAGGCTGTAGACAAATACTGG GAAGGCTGTAGACAAATACTGGG CTACAACCATCCCTTCAGACAGG TCTGATCCTGTCTGAAGGGATGG TTCTTCTGATCCTGTCTGAAGGG GTTCTTCTGATCCTGTCTGAAGG TCCTCTATTGTGTGCATCAAAGG TCCTTTGATGCACACAATAGAGG GGAAGCCTTAGATAAGATAGAGG TGACACAGGAAACAACAGCCAGG TAGGGTAATTTTGGCTGACCTGG CCCTATAGTGCAGAACCTCCAGG CCTATAGTGCAGAACCTCCAGGG CTATAGTGCAGAACCTCCAGGGG GCAGAACCTCCAGGGGCAAATGG GATGTACCATTTGCCCCTGGAGG CCAGGGGCAAATGGTACATCAGG GGTATTTAAATCTTGTGGGGTGG ATACCATGCTAAACACAGTGGGG TACCATGCTAAACACAGTGGGGG ACCATGCTAAACACAGTGGGGGG TCCCCCCACTGTGTTTAGCATGG TTCTGCAGCTTCCTCATTGATGG TCAATGAGGAAGCTGCAGAATGG CAATGAGGAAGCTGCAGAATGGG AGATTGCATCCAGTGCATGCAGG GATTGCATCCAGTGCATGCAGGG GCAATAGGCCCTGCATGCACTGG CATGCAGGGCCTATTGCACCAGG CTCATCTGGCCTGGTGCAATAGG CTTGGTTCTCTCATCTGGCCTGG CAGGCCAGATGAGAGAACCAAGG AGGCCAGATGAGAGAACCAAGGG GGCCAGATGAGAGAACCAAGGGG TTCCCCTTGGTTCTCTCATCTGG CCAAGGGGAAGTGACATAGCAGG AGGAACTACTAGTACCCTTCAGG AGTACCCTTCAGGAACAAATAGG CCCTTCAGGAACAAATAGGATGG TCCATCCTATTTGTTCCTGAAGG CATAATCCACCTATCCCAGTAGG ATTTCTCCTACTGGGATAGGTGG TAGATTTCTCCTACTGGGATAGG GTATAGCCCTACCAGCATTCTGG sequence: 628

sequence: 629

sequence: 641

sequence: 642

sequence:656

sequence: 672

sequence: 673

sequence: 679

sequence: 698

sequence:705

sequence: 753

sequence: 761

sequence: 772

sequence: 786

sequence:796

sequence:797

sequence:798

sequence:799

sequence: 815

sequence: 816

sequence: 838

sequence: 841

sequence: 884

sequence: 885

sequence:913

sequence:934

sequence:936

sequence:951

sequence: 952

sequence:979

sequence:985

sequence:989

sequence:990

sequence: 1040

sequence:1041

sequence:1083

sequence:1149

sequence:1167

sequence: 1185

sequence:1186

sequence:1187

sequence:1194

sequence: 1200

sequence: 1203

sequence:1325

sequence:1343

sequence:1344

sequence: 1345

sequence:1346

sequence: 1400

sequence:1403

sequence:1404

sequence:1429

sequence: 1430

sequence:1438

sequence:1444

sequence:1453

sequence:1462

sequence:1463

sequence:1464

sequence:1465

sequence:1467

sequence: 1480

sequence: 1500

sequence:1510

sequence:1514

sequence:1515

sequence:1543

sequence: 1549

sequence:1552

sequence:1617
LTR U5

LTR U5

50

45

45 
TTATGTCCAGAATGCTGGTAGGG CTTATGTCCAGAATGCTGGTAGG AGCATTCTGGACATAAGACAAGG GGACATAAGACAAGGACCAAAGG TAGTCTCTAAAGGGTTCCTTTGG CGGTCTACATAGTCTCTAAAGGG TCGGTCTACATAGTCTCTAAAGG AGCCGAGCAAGCTTCACAAGAGG TACCTCTTGTGAAGCTTGCTCGG TTGGATGACAGAAACCTTGTTGG GTTCGCATTTTGGACCAACAAGG TACAATCTGGGTTCGCATTTTGG ATTTCTTCTAGTGTCGCTCCTGG AGAAATGATGACAGCATGTCAGG GAAATGATGACAGCATGTCAGGG GATGACAGCATGTCAGGGAGTGG ATGACAGCATGTCAGGGAGTGGG TGACAGCATGTCAGGGAGTGGGG GACAGCATGTCAGGGAGTGGGGG ACAGCATGTCAGGGAGTGGGGGG TGTCAGGGAGTGGGGGGACCCGG AAAACTCTTGCTTTATGGCCGGG CAAAACTCTTGCTTTATGGCCGG CGGCCATAAAGCAAGAGTTTTGG CAGCCAAAACTCTTGCTTTATGG TGGTAGCTGGATTTGTTACTTGG GGCACATAGCCAAAAATTGCAGG GCACATAGCCAAAAATTGCAGGG CCAAAAATTGCAGGGCCCCTAGG TTGCAGGGCCCCTAGGAAAAAGG TGCAGGGCCCCTAGGAAAAAGGG CCCCTAGGAAAAAGGGCTGTTGG TCCAACAGCCCTTTTTCCTAGGG TTCCAACAGCCCTTTTTTCCTAGG AAAAAGGGCTGTTGGAAATGTGG GGGCTGTTGGAAATGTGGAAAGG TGTTGGAAATGTGGAAAGGAAGG GAAAGATTGTACTGAGAGACAGG GAAGATCTGGCCTTCCCACAAGG AAGATCTGGCCTTCCCACAAGGG TCTGGCCTTCCCACAAGGGAAGG CCTTCCCACAAGGGAAGGCCAGG CTTCCCACAAGGGAAGGCCAGGG AATTCCCTGGCCTTCCCTTGTGG CTGCTCTGAAGAAAATTCCCTGG TCTGGTGGGGCTGTTGGCTCTGG CTCTCTTCTGGTGGGGCTGTTGG CCCCACCAGAAGAGAGCTTCAGG ACCTGAAGCTCTCTTCTGGTGGG AACCTGAAGCTCTCTTCTGGTGG CCAGAAGAGAGCTTCAGGTTTGG CAGAAGAGAGCTTCAGGTTTGGG AGAAGAGAGCTTCAGGTTTGGGG AACAACTCCCTCTCAGAAGCAGG ATCGGCTCCTGCTTCTGAGAGGG TATCGGCTCCTGCTTCTGAGAGG GAAGCAGGAGCCGATAGACAAGG GGATACAGTTCCTTGTCTATCGG AGTGATCTGAGGGAAGCTAAAGG GCTTCCCTCAGATCACTCTTTGG GCTGCCAAAGAGTGATCTGAGGG CGCTGCCAAAGAGTGATCTGAGG CCCCTCGTCACAATAAAGATAGG CCCTCGTCACAATAAAGATAGGG CCTCGTCACAATAAAGATAGGGG CTCGTCACAATAAAGATAGGGGG TCGTCACAATAAAGATAGGGGGG AAAGATAGGGGGGCAATTAAAGG CCAAAAATGATAGGGGGAATTGG AAAATGATAGGGGGAATTGGAGG ATCTGCGGACATAAAGCTATAGG sequence: 1623

sequence: 1624

sequence: 1630

sequence:1638

sequence: 1654

sequence: 1663

sequence:1664

sequence: 1704

sequence: 1706

sequence: 1734

sequence: 1748

sequence: 1758

sequence: 1804

sequence:1821

sequence: 1822

sequence: 1827

sequence: 1828

sequence: 1829

sequence: 1830

sequence:1831

sequence:1837

sequence: 1855

sequence: 1856

sequence:1857

sequence: 1860

sequence:1893

sequence: 1985

sequence:1986

sequence:1994

sequence: 2001

sequence: 2002

sequence:2009

sequence: 2010

sequence: 2011

sequence: 2017

sequence: 2022

sequence: 2026

sequence:2058

sequence:2094

sequence: 2095

sequence:2099

sequence:2104

sequence: 2105

sequence:2109

sequence: 2122

sequence: 2146

sequence: 2152

sequence: 2159

sequence: 2160

sequence: 2161

sequence: 2164

sequence: 2165

sequence: 2166

sequence: 2196

sequence:2203

sequence:2204

sequence: 2211

sequence: 2221

sequence: 2242

sequence: 2248

sequence: 2252

sequence: 2253

sequence: 2277

sequence: 2278

sequence: 2279

sequence: 2280

sequence:2281

sequence: 2291

sequence: 2382

sequence: 2385

sequence: 2448
40 
CCTACACCTGTCAACATAATTGG ACTTTTGGGCCATCCATTCCTGG TTAGGAATACCACATCCTGCAGG TAGGAATACCACATCCTGCAGGG TGTTTTAACCCTGCAGGATGTGG ATCAGTAACAGTACTGGATGTGG TCAGTAACAGTACTGGATGTGGG TCAGTACAATGTGCTTCCACAGG CAGTACAATGTGCTTCCACAGGG ACAATGTGCTTCCACAGGGATGG GTGCTTCCACAGGGATGGAAAGG GGTGATCCTTTCCATCCCTGTGG ATGCTACACTGGAATATTGCTGG AACTGAGACAACATCTGTTGAGG TGAGACAACATCTGTTGAGGTGG GAGACAACATCTGTTGAGGTGGG AGACAACATCTGTTGAGGTGGGG AGAAAGAACCTCCATTCCTTTTG AGAACCTCCATTCCTTTGGATGG GAACCTCCATTCCTTTGGATGGG TCATAACCCATCCAAAGGAATGG GGAGTTCATAACCCATCCAAAGG GTACTGTCCATTTATCAGGATGG GGCTGTACTGTCCATTTATCAGG GCCTATAGTGCTGCCAGAAAAGG TCCTTTTCTGGCAGCACTATAGG ACAGTCCAGCTGTCCTTTTTCTGG TGGGCAAGTCAGATTTATGCAGG GGGCAAGTCAGATTTATGCAGGG AGCTCTGCTTCTTCTGTTAGTGG AGAAGAAGCAGAGCTAGAACTGG AGCTAGAACTGGCAGAAAACAGG GCTAGAACTGGCAGAAAACAGGG CAGGGAGATTCTAAAAGAACCGG TAGCAGAAATACAGAAGCAGGGG GAAATACAGAAGCAGGGGCAAGG AGAAGCAGGGGCAAGGCCAATGG AAAAGGAAACATGGGAAGCATGG AGGAAACATGGGAAGCATGGTGG AAGCATGGTGGACAGAGTATTGG CAGAGTATTGGCAAGCCACCTGG AAGCCACCTGGATTCCTGAGTGG AGCCACCTGGATTCCTGAGTGGG AAACTCCCACTCAGGAATCCAGG GTATTGACAAACTCCCACTCAGG CCCCTCCCTTAGTGAAGTTATGG ACCATAACTTCACTAAGGGAGGG TACCATAACTTCACTAAGGGAGG CTGGTACCATAACTTCACTAAGG ATGTAGATGGGGCAGCCAATAGG TGTAGATGGGGCAGCCAATAGGG ACAAAAAGTTGTCCCCCTAACGG GATTTGTTGTGTCCGTTAGGGGG TGATTTGTTGTGTCCGTTAGGGG CTGATTTGTTGTGTCCGTTAGGG TCTGATTTGTTGTGTCCGTTAGG AGCAATTCATCTAGCTTTGCAGG TCATCTAGCTTTGCAGGATTCGG CATCTAGCTTTGCAGGATTCGGG AGGAAAAAGTCTACCTGGCATGG GGAAAAAGTCTACCTGGCATGGG TGTGTGCTGGTACCCATGCCAGG CCAGCACACAAAGGAATTGGAGG GTAGATAAATTGGTCAGTGCTGG TCACAGTAATTGGAGAGCAATGG CTTTTGCTACTACAGGTGGTAGG TAGCTGACATTTATCACAGCTGG CTAAAAGGGGAAGCCATGCATGG GGACAAGTAGACTGTAGCCCAGG TAGACTGTAGCCCAGGAATATGG AATCTAGCTGCCATATTCCTGGG sequence: 2487

sequence: 2589

sequence: 2823

sequence: 2824

sequence: 2832

sequence: 2861

sequence: 2862

sequence: 2981

sequence:2982

sequence: 2986

sequence:2991

sequence: 2997

sequence:3018

sequence: 3160

sequence: 3163

sequence:3164

sequence:3165

sequence:3214

sequence: 3218

sequence: 3219

sequence: 3225

sequence: 3230

sequence: 3251

sequence: 3255

sequence: 3275

sequence: 3276

sequence: 3288

sequence: 3345

sequence: 3346

sequence: 3429

sequence: 3437

sequence: 3448

sequence: 3449

sequence: 3467

sequence: 3526

sequence:3531

sequence: 3538

sequence: 3730

sequence: 3733

sequence: 3745

sequence: 3757

sequence: 3769

sequence: 3770

sequence: 3775

sequence: 3783

sequence:3805

sequence:3806

sequence:3807

sequence:3811

sequence:3871

sequence: 3872

sequence: 3938

sequence:3950

sequence:3951

sequence: 3952

sequence:3953

sequence:3989

sequence:3995

sequence:3996

sequence: 4132

sequence: 4133

sequence: 4145

sequence:4158

sequence:4191

sequence: 4274

sequence: 4310

sequence: 4342

sequence:4362

sequence: 4383

sequence:4390

sequence: 4400 
CAATCTAGCTGCCATATTCCTGG GTAGCAGTTCATGTAGCCAGTGG GAAGTAATTCCAGCAGAGACAGG GTTTCTTGCCCTGTCTCTGCTGG GGCGGCCTTAACTGTAGTACTGG CTACAGTTAAGGCCGCCTGTTGG CAGTTAAGGCCGCCTGTTGGTGG AGTTAAGGCCGCCTGTTGGTGGG TAAGGCCGCCTGTTGGTGGGCGG AAGGCCGCCTGTTGGTGGGCGGG GATCCCCGCCCACCAACAGGCGG CTTGATCCCCGCCCACCAACAGG TTGGTGGGCGGGGATCAAGCAGG CCCTACAATCCCCAAAGTCAAGG TCCTTGACTTTGGGGATTGTAGG TATAGGACAGGTAAGAGATCAGG TAAAAGAAAAGGGGGGATTGGGG AAAAGAAAAGGGGGGATTGGGGG AAAGAAAAGGGGGGATTGGGGGG GGGATTGGGGGGTACAGTGCAGG GGATTGGGGGGTACAGTGCAGGG GATTGGGGGGTACAGTGCAGGGG GGGACAGCAGAGATCCAGTTTGG AGCAGAGATCCAGTTTGGAAAGG TTTGCTGGTCCTTTCCAAACTGG AAGGACCAGCAAAGCTCCTCTGG CCAGCAAAGCTCCTCTGGAAAGG AAGCTCCTCTGGAAAGGTGAAGG AGCTCCTCTGGAAAGGTGAAGGG GCTCCTCTGGAAAGGTGAAGGGG CTGCCCCTTCACCTTTCCAGAGG GCAAAGATCATCAGGGATTATGG GATTATGGAAAACAGATGGCAGG TGATTGTGTGGCAAGTAGACAGG TGTGGCAAGTAGACAGGATGAGG ACAGGATGAGGATTAACACATGG TTTCAAGGAAAGCTAAGGACTGG TCAGAAGTACACATCCCACTAGG CAGAAGTACACATCCCACTAGGG AGAAGTACACATCCCACTAGGGG CTAATTTAGCATCCCCTAGTGGG ACTAATTTAGCATCCCCTAGTGG ACATATTGGGGTCTGCATACAGG TGCATACAGGAGAAAGAGACTGG GTCAGGGAGTCTCCATAGAATGG AGGGAGTCTCCATAGAATGGAGG TTAGTTGGTCTGCTAGGTCAGGG ATTAGTTGGTCTGCTAGGTCAGG GATGAATTAGTTGGTCTGCTAGG TAGGACGTATAGTTAGTCCTAGG CCTAGGTGTGAATATCAAGCAGG CAAGCAGGACATAACAAGGTAGG GGTAGGATCTCTACAGTACTTGG AGCCACCTTTGCCTAGTGTTAGG TTCCTAACACTAGGCAAAGGTGG AGTTTCCTAACACTAGGCAAAGG TCTGTCAGTTTCCTAACACTAGG GGAAACTGACAGAGGACAGATGG GAACAAGCCCCAGAAGACCAAGG AACAAGCCCCAGAAGACCAAGGG CTGTGGCCCTTGGTCTTCTGGGG TCTGTGGCCCTTGGTCTTCTGGG CTCTGTGGCCCTTGGTCTTCTGG AGAAGACCAAGGGCCACAGAGGG ATGGCTCCCTCTGTGGCCCTTGG TCATTGTATGGCTCCCTCTGTGG AGAGGGAGCCATACAATGAATGG ATGGACACTAGAGCTTTTAGAGG AAGTTATGGAGCCATATCCTAGG AGGATATGGCTCCATAACTTAGG ATGAAACTTACGGGGATACTTGG sequence:4401 sequence:4452 sequence: 4488 sequence:4497 sequence: 4594 sequence: 4600 sequence:4603 sequence:4604 sequence:4607 sequence:4608 sequence:4612 sequence:4615 sequence:4619 sequence:4653 sequence:4654 sequence:4712 sequence:4784 sequence:4785 sequence:4786 sequence:4797 sequence:4798 sequence:4799 sequence:4912 sequence: 4917 sequence:4926 sequence:4936 sequence:4941 sequence:4947 sequence:4948 sequence:4949 sequence:4952 sequence:5022 sequence:5037 sequence:5063 sequence:5069 sequence:5081 sequence:5132 sequence:5197 sequence:5198 sequence:5199 sequence:5211 sequence:5212 sequence:5242 sequence:5255 sequence:5285 sequence:5288 sequence:5337 sequence:5338 sequence:5343 sequence:5414 sequence:5431 sequence:5446 sequence:5463 sequence:5519 sequence:5521 sequence:5524 sequence:5530 sequence:5540 sequence:5562 sequence:5563 sequence:5569 sequence:5570 sequence:5571 sequence:5573 sequence:5579 sequence:5586 sequence:5590 sequence:5609 sequence:5661 sequence:5664 sequence:5698
Pol

Pol Pol Pol Pol Pol Pol Pol Pol Pol Pol Pol Pol Pol Pol Pol Pol Pol cPPT Pol cPPT

Pol Pol Pol

Pol Pol Pol Pol Pol Pol Pol Pol Pol Pol Pol Pol Vif Pol Vif Pol Vif Vif Vif Vif Vif Vif Vif Vif Vif Vif Vif Vif Vif Vif Vif Vif Vif Vif Vif Vif Vif Vif Vif Vif Vpr Vif Vpr Vif Vpr Vif Vpr Vif Vpr Vif Vpr Vif Vpr Vif Vpr Vif Vpr Vif Vpr 
TGAAACTTACGGGGATACTTGGG ACTTACGGGGATACTTGGGCAGG CGGGGATACTTGGGCAGGAGTGG GTCGACACCCAATTCTGAAATGG GGGTGTCGACATAGCAGAATAGG GAATAGGCGTTACTCGACAGAGG CGACAGAGGAGAGCAAGAAATGG GGCTCTAGTCTAGGATCTACTGG TAGATCCTAGACTAGAGCCCTGG TGCTTCCAGGGCTCTAGTCTAGG CTAGAGCCCTGGAAGCATCCAGG TGACTTCCTGGATGCTTCCAGGG CTGACTTCCTGGATGCTTCCAGG GCAGTTTTAGGCTGACTTCCTGG AAAGCCTTAGGCATCTCCTATGG CCTTAGGCATCTCCTATGGCAGG TCTCCTATGGCAGGAAGAAGCGG TCTCCGCTTCTTCCTGCCATAGG ATAGAAAGAGCAGAAGACAGTGG GACAGTGGCAATGAGAGTGAAGG AGGAGAAGTATCAGCACTTGTGG AGTATCAGCACTTGTGGAGATGG GTATCAGCACTTGTGGAGATGGG TATCAGCACTTGTGGAGATGGGG ATCAGCACTTGTGGAGATGGGGG AGCACTTGTGGAGATGGGGGTGG AAATGGGGCACCATGCTCCTTGG AATGGGGCACCATGCTCCTTGGG CATCAATATCCCAAGGAGCATGG GGGAGCAGCAGGAAGCACTATGG GGAGCAGCAGGAAGCACTATGGG CGCAGCGTCAATGACGCTGACGG GTCAATGACGCTGACGGTACAGG GCAGCAGAACAATTTGCTGAGGG CAATTTGCTGAGGGCTATTGAGG ATCTGTTGCAACTCACAGTCTGG TCTGTTGCAACTCACAGTCTGGG CTGTTGCAACTCACAGTCTGGGG CTGGGGCATCAAACAGCTCCAGG ACAGCTCCAGGCAAGAATCCTGG CCAGGCAAGAATCCTGGCTGTGG TTAGGTATCTTTCCACAGCCAGG GGCTGTGGAAAGATACCTAAAGG ACCCACCTCCCAATCCCGAGGGG TCCCCTCGGGATTGGGAGGTGGG GTCCCCTCGGGATTGGGAGGTGG TGTCGGGTCCCCTCGGGATTGGG CTGTCGGGTCCCCTCGGGATTGG CAATCCCGAGGGGACCCGACAGG TCTATTCCTTCGGGCCTGTCGGG TTCTATTCCTTCGGGCCTGTCGG CCCGAAGGAATAGAAGAAGAAGG GGATCCGTTCACTAATCGAATGG ACGGATCCTTAGCACTTATCTGG CGGATCCTTAGCACTTATCTGGG ATCGTCCCAGATAAGTGCTAAGG CACTTATCTGGGACGATCTGCGG GGTGGTAGCTGAAGAGGCACAGG TCAAGCGGTGGTAGCTGAAGAGG AGAGTAAGTCTCTCAAGCGGTGG TCAAGAGTAAGTCTCTCAAGCGG CTTGATTGTAACGAGGATTGTGG AACGAGGATTGTGGAACTTCTGG ACGAGGATTGTGGAACTTCTGGG TTGTGGAACTTCTGGGACGCAGG TGTGGAACTTCTGGGACGCAGGG GTGGAACTTCTGGGACGCAGGGG TGGAACTTCTGGGACGCAGGGGG ACTTCTGGGACGCAGGGGGTGGG GGTGGGAAGCCCTCAAATATTGG GGGAAGCCCTCAAATATTGGTGG sequence:5699

sequence: 5703

sequence: 5708

sequence: 5768

sequence: 5781

sequence:5797

sequence:5811

sequence:5836

sequence:5840

sequence: 5845

sequence:5851

sequence: 5857

sequence:5858

sequence: 5869

sequence: 5950

sequence:5954

sequence:5963

sequence:5966

sequence:6196

sequence:6211

sequence: 6231

sequence:6237

sequence:6238

sequence: 6239

sequence: 6240

sequence: 6243

sequence: 6266

sequence:6267

sequence: 6276

sequence: 7783

sequence:7784

sequence: 7807

sequence: 7813

sequence:7864

sequence: 7873

sequence:7905

sequence:7906

sequence: 7907

sequence: 7924

sequence:7936

sequence:7942

sequence:7954

sequence: 7957

sequence:8369

sequence: 8370

sequence: 8371

sequence: 8377

sequence: 8378

sequence: 8379

sequence:8393

sequence:8394

sequence: 8402

sequence: 8448

sequence: 8463

sequence:8464

sequence: 8469

sequence: 8475

sequence: 8500

sequence: 8506

sequence: 8518

sequence:8521

sequence: 8539

sequence: 8548

sequence: 8549

sequence: 8556

sequence: 8557

sequence: 8558

sequence: 8559

sequence: 8563

sequence: 8580

sequence: 8583

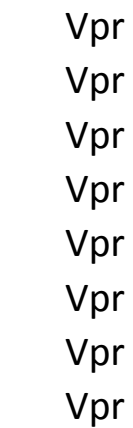

Vpr 5 'Tat

Vpr 5' Tat

5' Tat

5' Tat

5 ' Tat

5' Tat

5' Tat

5' Tat

5 ' Tat

5' Tat 
GGAGATTCCACCAATATTTGAGG TCTCCTACAGTATTGGAGTCAGG GTTCCTGACTCCAATACTGTAGG TGATTGGATGGCCTGCTGTAAGG GATTGGATGGCCTGCTGTAAGGG CTCATTCTTTCCCTTACAGCAGG CGAGCTGAGCCAGCAGCAGATGG GAGCTGAGCCAGCAGCAGATGGG AGCTGAGCCAGCAGCAGATGGGG TGAGCCAGCAGCAGATGGGGTGG GCTCCCACCCCATCTGCTGCTGG TCTCGAGACCTAGAAAAACATGG CTAACAATGCTGCTTGTGCCTGG TGCCTGGCTAGAAGCACAAGAGG CTCCTCTTGTGCTTCTAGCCAGG CTGGCTAGAAGCACAAGAGGAGG GCACAAGAGGAGGAAGAGGTGGG GGGTTTTCCAGTCACACCTCAGG AAAGGTACCTGAGGTGTGACTGG ATTGGTCTTAAAGGTACCTGAGG AAAGAAAAGGGGGGACTGGAAGG AAGAAAAGGGGGGACTGGAAGGG GACAAGATATCCTTGATCTGTGG GAACTACACACCAGGGCCAGGGG GGATATCTGACCCCTGGCCCTGG GTCAGTGGATATCTGACCCCTGG GTCAGATATCCACTGACCTTTGG TAGCTTGTAGCACCATCCAAAGG AGTACCAGTTGAGCCAGATAAGG TCTACCTTATCTGGCTCAACTGG TGAGCCAGATAAGGTAGAAGAGG TTGGCCTCTTCTACCTTATCTGG AAGGTAGAAGAGGCCAATAAAGG GCTGGTGTTCTCTCCTTTATTGG GCTCACAGGGTGTAACAAGCTGG TTACACCCTGTGAGCCTGCATGG CCCTGTGAGCCTGCATGGAATGG TCCATTCCATGCAGGCTCACAGG CAGGGTCATCCATTCCATGCAGG ACTCTAACACTTCTCTCTCAGGG CTGAGAGAGAAGTGTTAGAGTGG AGAGAGAAGTGTTAGAGTGGAGG CCGCCTAGCATTTCATCACGTGG GGGCCACGTGATGAAATGCTAGG CGTGGCCCGAGAGCTGCATCCGG CAGCAGTTCTTGAAGTACTCCGG CTGACATCGAGCTTGCTACAAGG TGACATCGAGCTTGCTACAAGGG TGCTACAAGGGACTTTCCGCTGG GCTACAAGGGACTTTCCGCTGGG GGGGACTTTCCAGGGAGGCGTGG CTTTCCAGGGAGGCGTGGCCTGG TTTCCAGGGAGGCGTGGCCTGGG TGCTTATATGCAGCATCTGAGGG CTGCTTATATGCAGCATCTGAGG CACACTACTTGAAGCACTCAAGG sequence: 8590 sequence: 8608 sequence: 8611 sequence:8815 sequence: 8816 sequence: 8826 sequence:8850 sequence: 8851 sequence: 8852 sequence:8855 sequence: 8859 sequence: 8886 sequence: 8935 sequence: 8951 sequence: 8953 sequence: 8954 sequence: 8964 sequence: 8984 sequence:8991 sequence:9000 sequence:9060 sequence:9061 sequence:9103 sequence:9161 sequence:9171 sequence:9177 sequence: 9183 sequence:9199 sequence:9221 sequence: 9225 sequence:9230 sequence:9234 sequence: 9240 sequence:9253 sequence:9271 sequence:9279 sequence:9284 sequence:9285 sequence:9293 sequence:9311 sequence:9313 sequence:9316 sequence:9347 sequence:9350 sequence:9365 sequence:9384 sequence:9404 sequence:9405 sequence:9417 sequence:9418 sequence:9438 sequence: 9443 sequence:9444 sequence:9487 sequence: 9488 sequence:9613

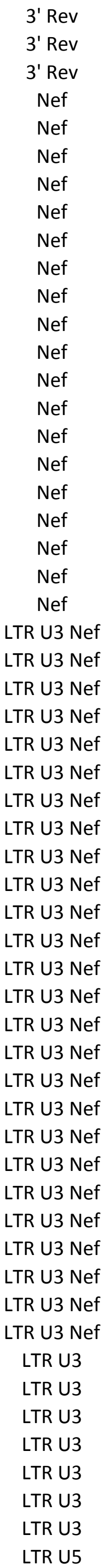




\begin{tabular}{|c|c|c|c|c|}
\hline BK132 Target sequence & 5' end of gRNA target site & Strand & \% GC content & HIV-1 target \\
\hline $\begin{array}{l}\text { CTGGTTTCTCTTTCGCTTTCAGG } \\
\text {. }\end{array}$ & sequence:4 & - & 45 & \\
\hline GAAAGCGAAAGAGAAACCAGAGG & sequence:7 & + & 45 & \\
\hline CTGCGTCGAGAGAGCTCCTCTGG & sequence:23 & - & 65 & \\
\hline CAGAGGAGCTCTCTCGACGCAGG & sequence:24 & + & 65 & \\
\hline AGCTCTCTCGACGCAGGACTCGG & sequence:30 & + & 60 & \\
\hline TCGGCTTGCTGAAGCGCGCACGG & sequence:49 & + & 65 & \\
\hline GCTGAAGCGCGCACGGCAAGAGG & sequence:56 & + & 70 & \\
\hline CCAAAAATTTTGACTAGCGGAGG & sequence:104 & + & 40 & \\
\hline TTTGACTAGCGGAGGCTAGAAGG & sequence:112 & + & 50 & \\
\hline GAGGCTAGAAGGAGAGAGATGGG & sequence:123 & + & 50 & Gag \\
\hline GCGAGAGCGTCAGTATTAAGCGG & sequence:147 & + & 50 & Gag \\
\hline CGAGAGCGTCAGTATTAAGCGGG & sequence:148 & + & 50 & Gag \\
\hline GAGAGCGTCAGTATTAAGCGGGG & sequence:149 & + & 50 & Gag \\
\hline AGAGCGTCAGTATTAAGCGGGGG & sequence:150 & + & 50 & Gag \\
\hline GCGGGGGACAATTAGATAGATGG & sequence:166 & + & 50 & Gag \\
\hline CGGGGGACAATTAGATAGATGGG & sequence:167 & + & 45 & Gag \\
\hline GATGGGAGAAAATTCGGTTACGG & sequence:184 & + & 40 & Gag \\
\hline GAGAAAATTCGGTTACGGCCAGG & sequence:189 & + & 50 & Gag \\
\hline AGAAAATTCGGTTACGGCCAGGG & sequence:190 & + & 45 & Gag \\
\hline GAAAATTCGGTTACGGCCAGGGG & sequence:191 & + & 50 & Gag \\
\hline AAAATTCGGTTACGGCCAGGGGG & sequence:192 & + & 50 & Gag \\
\hline AACATATAGTATGGGCAAGCAGG & sequence:235 & + & 40 & Gag \\
\hline ACATATAGTATGGGCAAGCAGGG & sequence:236 & + & 40 & Gag \\
\hline GAACGATTCGCAGTTAATCCTGG & sequence:264 & + & 45 & Gag \\
\hline CGCAGTTAATCCTGGCCTATTGG & sequence: 272 & + & 50 & Gag \\
\hline TCTGATGTTTCCAATAGGCCAGG & sequence:282 & - & 45 & Gag \\
\hline GGCCTATTGGAAACATCAGAAGG & sequence: 285 & + & 45 & Gag \\
\hline AGCCTTCTGATGTTTCCAATAGG & sequence: 287 & - & 40 & Gag \\
\hline AGAAGGCTGTAGACAAATACTGG & sequence:302 & + & 40 & Gag \\
\hline GAAGGCTGTAGACAAATACTGGG & sequence:303 & + & 40 & Gag \\
\hline CTACAACCAAGCCTTCAGACAGG & sequence:330 & + & 50 & Gag \\
\hline TCTGATCCTGTCTGAAGGCTTGG & sequence:336 & - & 50 & Gag \\
\hline GTTCTTCTGATCCTGTCTGAAGG & sequence:341 & - & 45 & Gag \\
\hline GATAGAGGTAAAAGACACCAAGG & sequence:413 & + & 40 & Gag \\
\hline GGAAGCTTTAGAGAAGATAGAGG & sequence:434 & + & 40 & Gag \\
\hline TAGGGTAATTTTGGGTAACCTGG & sequence:518 & - & 40 & Gag \\
\hline CCCTATAGTGCAAAATATGCAGG & sequence:536 & + & 40 & Gag \\
\hline GCAAAATATGCAGGGGCAAATGG & sequence:545 & + & 45 & Gag \\
\hline GCAGGGGCAAATGGTACATCAGG & sequence:554 & + & 55 & Gag \\
\hline GGTGTTTAAATCTTGTGGGGTGG & sequence:676 & - & 45 & Gag \\
\hline AAACACCATGCTAAACACAGTGG & sequence:692 & + & 40 & Gag \\
\hline AACACCATGCTAAACACAGTGGG & sequence:693 & + & 40 & Gag \\
\hline ACACCATGCTAAACACAGTGGGG & sequence:694 & + & 45 & Gag \\
\hline CACCATGCTAAACACAGTGGGGG & sequence:695 & + & 50 & Gag \\
\hline ACCATGCTAAACACAGTGGGGGG & sequence:696 & + & 50 & Gag \\
\hline TCCCCCCACTGTGTTTAGCATGG & sequence:697 & - & 55 & Gag \\
\hline TTCTGCAGCTTCCTCATTGATGG & sequence:751 & - & 45 & Gag \\
\hline TCAATGAGGAAGCTGCAGAATGG & sequence: 754 & + & 45 & Gag \\
\hline CAATGAGGAAGCTGCAGAATGGG & sequence:755 & + & 45 & Gag \\
\hline AGATTGCATCCAGTGCATGCAGG & sequence:780 & + & 50 & Gag \\
\hline GATTGCATCCAGTGCATGCAGGG & sequence:781 & + & 50 & Gag \\
\hline GCAATAGGCCCTGCATGCACTGG & sequence:789 & - & 60 & Gag \\
\hline CATGCAGGGCCTATTGCACCAGG & sequence:795 & + & 60 & Gag \\
\hline СTCATCTGGCCTGGTGCAATAGG & sequence:804 & - & 55 & Gag \\
\hline CTTGGTTCTCTCATCTGGCCTGG & sequence:813 & - & 55 & Gag \\
\hline CAGGCCAGATGAGAGAACCAAGG & sequence: 814 & + & 55 & Gag \\
\hline AGGCCAGATGAGAGAACCAAGGG & sequence: 815 & + & 50 & Gag \\
\hline GGCCAGATGAGAGAACCAAGGGG & sequence:816 & + & 55 & Gag \\
\hline TTCCCCTTGGTTCTCTCATCTGG & sequence: 818 & - & 50 & Gag \\
\hline CCAAGGGGAAGTGACATAGCAGG & sequence:831 & + & 55 & Gag \\
\hline AGGAACTACTAGTACCCTTCAGG & sequence: 851 & + & 45 & Gag \\
\hline AGTACCCTTCAGGAACAAATAGG & sequence:861 & + & 40 & Gag \\
\hline CCCTTCAGGAACAAATAGGATGG & sequence:865 & + & 45 & Gag \\
\hline TCCATCCTATTTGTTCCTGAAGG & sequence:866 & - & 40 & Gag \\
\hline ACATAATCCACCTATCCCAGTGG & sequence:893 & + & 45 & Gag \\
\hline САTAАTCCACСTATCCCAGTGGG & sequence:894 & + & 45 & Gag \\
\hline ATTTCTCCCACTGGGATAGGTGG & sequence:900 & - & 50 & Gag \\
\hline TAAATTTCTCCCACTGGGATAGG & sequence:903 & - & 40 & Gag \\
\hline GTATAGCCCTACCAGCATTTTGG & sequence:968 & + & 45 & Gag \\
\hline GGACATAAGACAAGGACCAAAGG & sequence:989 & + & 45 & Gag \\
\hline
\end{tabular}


TAGTCTCTAAAGGGTTCCTTTGG CGGTCTACATAGTCTCTAAAGGG CCTTTAGAGACTATGTAGACCGG AAGAGCCGAGCAAGCCTCACAGG AGCCGAGCAAGCCTCACAGGAGG TACCTCCTGTGAGGCTTGCTCGG CCTCACAGGAGGTAAAAAATTGG TTGGATGACAGAAACCTTGTTGG GTTCGCATTTTGGACCAACAAGG TACAATCTGGGTTCGCATTTTGG ATTTCTTCTAGTGTAGCTGCTGG AGAAATGATGACAGCATGTCAGG GAAATGATGACAGCATGTCAGGG GATGACAGCATGTCAGGGAGTGG ATGACAGCATGTCAGGGAGTGGG ACAGCATGTCAGGGAGTGGGAGG TGTCAGGGAGTGGGAGGTCCCGG AGTGGGAGGTCCCGGCCATAAGG AAAACTCTTGCCTTATGGCCGGG CAAAACTCTTGCCTTATGGCCGG CGGCCATAAGGCAAGAGTTTTGG CCATAAGGCAAGAGTTTTGGCGG TGGTAGCTGAATTTGTCACTTGG GCTACCATAATGATGCAGAAAGG GGCACATAGCCCGAAATTGCAGG GCACATAGCCCGAAATTGCAGGG CCCGAAATTGCAGGGCCCCTAGG TCCTAGGGGCCCTGCAATTTCGG TTGCAGGGCCCCTAGGAAGAAGG TGCAGGGCCCCTAGGAAGAAGGG TCCAACAGCCCTTCTTCCTAGGG CTCCAACAGCCCTTCTTCCTAGG GGGCTGTTGGAGATGTGGAAAGG GAAAATCTGGCCTTCCCACAAGG AAAATCTGGCCTTCCCACAAGGG TCTGGCCTTCCCACAAGGGAAGG CCTTCCCACAAGGGAAGGCCAGG CTTCCCACAAGGGAAGGCCAGGG AATTCCCTGGCCTTCCCTTGTGG CTGCTCTGAAGAAAATTCCCTGG CTCTCTGCTGGTGGGGCTGTCGG CCCCACCAGCAGAGAGCTTCAGG CCAGCAGAGAGCTTCAGGTTTGG CAGCAGAGAGCTTCAGGTTTGGG AGCAGAGAGCTTCAGGTTTGGGG AACAACTCCCTCTCAGAAGCAGG ATTGTCTCCTGCTTCTGAGAGGG TATTGTCTCCTGCTTCTGAGAGG GAAGCAGGAGACAATAGACAAGG AGTGATTTGAGGGCAGTTAAAGG ACTGCCCTCAAATCACTCTTTGG GTTGCCAAAGAGTGATTTGAGGG CCCCTCGTCACAATAAAGATAGG CCCTCGTCACAATAAAGATAGGG CCTCGTCACAATAAAGATAGGGG CTCGTCACAATAAAGATAGGGGG TCGTCACAATAAAGATAGGGGGG AAAGATAGGGGGGCAACTAAAGG GGAAGCTCTATTAGATACAGGGG CCAAAAATGATAGGGGGAATTGG AAAATGATAGGGGGAATTGGAGG CCTACACCTGTCAACATAATTGG ACTTTTGGGCCATCCATTCCTGG GACTTCTGGGAAGTTCAATTAGG TTAGGAATACCACATCCCGCAGG TAGGAATACCACATCCCGCAGGG TTTTTCAACCCTGCGGGATGTGG ATCAGTAACGGTACTGGATGTGG TCAGTAACGGTACTGGATGTGGG CTCATTGTTTGTACTCGGTATGG GGTGTCTCATTGTTTGTACTCGG sequence:1005 sequence:1014 sequence: 1015 sequence:1052 sequence: 1055 sequence: 1057 sequence:1066 sequence: 1085 sequence:1099 sequence:1109 sequence: 1155 sequence:1172 sequence: 1173 sequence: 1178 sequence: 1179 sequence:1182 sequence: 1188 sequence:1196 sequence:1206 sequence: 1207 sequence: 1208 sequence:1211 sequence: 1244 sequence: 1260 sequence:1336 sequence: 1337 sequence:1345 sequence: 1346 sequence:1352 sequence:1353 sequence:1361 sequence:1362 sequence: 1373 sequence:1445 sequence: 1446 sequence: 1450 sequence:1455 sequence:1456 sequence: 1460 sequence:1473 sequence:1503 sequence: 1510 sequence: 1515 sequence:1516 sequence:1517 sequence: 1547 sequence:1554 sequence: 1555 sequence: 1562 sequence:1593 sequence:1599 sequence:1603 sequence:1628 sequence:1629 sequence:1630 sequence:1631 sequence:1632 sequence:1642 sequence:1663 sequence: 1733 sequence:1736 sequence:1838 sequence: 1940 sequence: 2156 sequence: 2174 sequence: 2175 sequence: 2183 sequence: 2212 sequence: 2213 sequence: 2292 sequence: 2297 
AGTACAAACAATGAGACACCAGG GTACAAACAATGAGACACCAGGG TCAGTACAATGTGCTTCCACAGG CAGTACAATGTGCTTCCACAGGG ACAATGTGCTTCCACAGGGATGG GTGCTTCCACAGGGATGGAAAGG GGTGATCCTTTCCATCCCTGTGG AACTGAGACAACATCTGTTGAGG TGAGACAACATCTGTTGAGGTGG GAGACAACATCTGTTGAGGTGGG AGACAACATCTGTTGAGGTGGGG AGAAAGAACCTCCATTCCTTTGG AGAACCTCCATTCCTTTGGATGG GAACCTCCATTCCTTTGGATGGG TCATAACCCATCCAAAGGAATGG GGAGTTCATAACCCATCCAAAGG GTACTGTCCATTTATCAGGATGG GGTTGTACTGTCCATTTATCAGG AGTACAACCTATAGTGCTGCCGG TCTTTTTCCGGCAGCACTATAGG TGCTGCCGGAAAAAGACAGCTGG ACAGTCCAGCTGTCTTTTTTCCGG TGGGCAAGTCAGATTTACCCAGG GGGCAAGTCAGATTTACCCAGGG TTAGTGCTTTGGTTCCCCTAAGG AGCTCTGCCTCTTTTGTTAGTGG AAAAGAGGCAGAGCTAGAACTGG AGCTAGAACTGGCAGAAAACAGG GCTAGAACTGGCAGAAAACAGGG TAGCAGAAATACAGAAGCAGGGG GAAATACAGAAGCAGGGGCAAGG AGAAGCAGGGGCAAGGCCAATGG AAGAAACATGGGAAACATGGTGG AAACATGGTGGACAGAGTATTGG CAGAGTATTGGCAAGCCACCTGG AAGCCACCTGGATTCCTGAGTGG AGCCACCTGGATTCCTGAGTGGG AAACTCCCACTCAGGAATCCAGG GTATTGACAAACTCCCACTCAGG CCCCTCCCTTAGTGAAATTATGG CTATGGGCTCTTTTTTCTAACTGG AAGGTTTCTGCTCCTACTATGGG GAAGGTTTCTGCTCCTACTATGG GCAGAAACCTTCTATGTAGATGG AACCTTCTATGTAGATGGGGCGG AGCCGCCCCATCTACATAGAAGG ATGTAGATGGGGCGGCTAACAGG TGTAGATGGGGCGGCTAACAGGG GCTAACAGGGAGACTAAATTAGG GCAGGATATGTGACTAACAGAGG AGCAATTCACCTAGCTTTGCAGG TCACCTAGCTTTGCAGGATTCGG CACCTAGCTTTGCAGGATTCGGG ATCCCGAATCCTGCAAAGCTAGG ACAAAAGGAAAAGGTCTACCTGG AGGAAAAGGTCTACCTGGCATGG GGAAAAGGTCTACCTGGCATGGG TGTGTGCTGGTACCCATGCCAGG GGCATGGGTACCAGCACACAAGG GCATGGGTACCAGCACACAAGGG GTACCAGCACACAAGGGAATTGG CCAGCACACAAGGGAATTGGAGG AATTAGTCAGTGCTGGAATCAGG TCACAGTAATTGGAGAGCAATGG CTTTTGCTACTACAGGTGGCAGG TAGCTGACATTTATCACAGCTGG CTAAAAGGAGAAGCCATGCATGG AGGAGAAGCCATGCATGGACAGG ACAGTCTACCTGTCCATGCATGG GGACAGGTAGACTGTAGTCCAGG TAGACTGTAGTCCAGGAATATGG sequence: 2300

sequence: 2301

sequence: 2332

sequence: 2333

sequence: 2337

sequence: 2342

sequence: 2348

sequence: 2511

sequence: 2514

sequence: 2515

sequence: 2516

sequence: 2565

sequence: 2569

sequence: 2570

sequence: 2576

sequence: 2581

sequence: 2602

sequence: 2606

sequence: 2620

sequence: 2627

sequence: 2634

sequence: 2639

sequence: 2696

sequence: 2697

sequence: 2746

sequence: 2780

sequence: 2788

sequence: 2799

sequence: 2800

sequence: 2877

sequence: 2882

sequence: 2889

sequence:3084

sequence:3096

sequence: 3108

sequence: 3120

sequence:3121

sequence:3126

sequence:3134

sequence:3156

sequence: 3181

sequence:3197

sequence:3198

sequence:3209

sequence: 3214

sequence:3216

sequence: 3222

sequence:3223

sequence: 3236

sequence: 3263

sequence: 3340

sequence: 3346

sequence: 3347

sequence:3349

sequence: 3478

sequence: 3483

sequence:3484

sequence:3496

sequence:3499

sequence: 3500

sequence: 3506

sequence:3509

sequence: 3549

sequence: 3625

sequence: 3661

sequence: 3693

sequence: 3713

sequence: 3718

sequence: 3726

sequence: 3734

sequence: 3741
40

40

45

45

50

55

55

40

45

45

45

40

45

45

40

45

40

40

45

45

55

45

50

50

45

45

45

45

45

40

50

60

40

40

55

55

55

50

45

45

40

40

45

40

45

55

55

55

40

45

45

45

50

50

40

50

50

60

60

55

50

55

40

40

50

40

45

55

50

55

40
Pol

Pol Pol Pol Pol Pol Pol Pol Pol Pol Pol Pol Pol Pol Pol Pol Pol Pol Pol Pol Pol Pol Pol Pol Pol Pol Pol Pol Pol Pol Pol Pol Pol Pol Pol Pol Pol Pol Pol Pol Pol Pol Pol Pol Pol Pol Pol Pol Pol Pol Pol Pol Pol Pol Pol Pol Pol Pol Pol Pol Pol Pol Pol Pol Pol Pol Pol Pol Pol Pol Pol 
CAATCTAGTTGCCATATTCCTGG

TGGCTACATGAACTGCTACCAGG GTAGCAGTTCATGTAGCCAGTGG GAAGTTATTCCAGCAGAGACAGG AAGTTATTCCAGCAGAGACAGGG TATTCCAGCAGAGACAGGGCAGG GTTTCCTGCCCTGTCTCTGCTGG CAGCAATTTCACCAGTACTACGG TTTCACCAGTACTACGGTTAAGG GGCAGCCTTAACCGTAGTACTGG CTACGGTTAAGGCTGCCTGTTGG CGGTTAAGGCTGCCTGTTGGTGG GGTTAAGGCTGCCTGTTGGTGGG TAAGGCTGCCTGTTGGTGGGCGG AAGGCTGCCTGTTGGTGGGCGGG AGGCTGCCTGTTGGTGGGCGGGG CTTGATCCCCGCCCACCAACAGG TTGGTGGGCGGGGATCAAGCAGG CTTGACTTTGGGGATTGTAGGGG CCCTACAATCCCCAAAGTCAAGG TCCTTGACTTTGGGGATTGTAGG TCTACTACTCCTTGACTTTGGGG TATAGGACAGGTAAGAGATCAGG TAAAAGAAAAGGGGGGATTGGGG AAAAGAAAAGGGGGGATTGGGGG AAAGAAAAGGGGGGATTGGGGGG GGGATTGGGGGGTACAGTGCAGG GGATTGGGGGGTACAGTGCAGGG GATTGGGGGGTACAGTGCAGGGG GGGACAGCAGAGATCCACTTTGG AGCAGAGATCCACTTTGGAAAGG TTTGCTGGTCCTTTCCAAAGTGG AAGGACCAGCAAAGCTTCTGTGG CCAGCAAAGCTTCTGTGGAAAGG AGCTTCTGTGGAAAGGTGAAGGG GCTTCTGTGGAAAGGTGAAGGGG GATTATGGAAAACAGATGGCAGG TGATTGTGTGGCAAGTAGACAGG TGTGGCAAGTAGACAGGATGAGG TCAGAAGTACATATCCCACTAGG AGTACATATCCCACTAGGAGAGG CCCACTAGGAGAGGCTAGCTTGG ACCAAGCTAGCCTCTCCTAGTGG ACATATTGGGGTCTGCATACAGG TGCATACAGGAGAAAGAGAATGG AGGAGAAAGAGAATGGCATTTGG AGAGAATGGCATTTGGGTCAAGG GTCAAGGAGTCTCCATAGAATGG AAGGAGTCTCCATAGAATGGAGG GTCTCCATAGAATGGAGGAAAGG TCTTCCTTTCCTCCATTCTATGG TATAACACACAAGTAGACCCAGG TTAGTTGGTCTGCTAGGCCTGGG ATTAGTTGGTCTGCTAGGCCTGG GATGAATTAGTTGGTCTGCTAGG CCTAGTTGTGAATATCAAGCAGG CAAGCAGGACATAACAAGGTAGG GGTAGGATCTCTACAGTACTTGG AGCCACCTTTGCCTAGTGTTAGG TTCCTAACACTAGGCAAAGGTGG AGTTTCCTAACACTAGGCAAAGG TCTGTCAGTTTCCTAACACTAGG GGAAACTGACAGAGGATAGATGG GAACAAGCCCCAGAAGACCAAGG AACAAGCCCCAGAAGACCAAGGG CTGTGGCCCTTGGTCTTCTGGGG TCTGTGGCCCTTGGTCTTCTGGG CTCTGTGGCCCTTGGTCTTCTGG ATGGCTCTCTCTGTGGCCCTTGG TCATTGTATGGCTCTCTCTGTGG AGAGAGAGCCATACAATGAATGG sequence: 3752 sequence: 3799 sequence:3803 sequence: 3839 sequence: 3840 sequence: 3844 sequence: 3848 sequence: 3934 sequence: 3940 sequence: 3945 sequence:3951 sequence: 3954 sequence:3955 sequence: 3958 sequence:3959 sequence:3960 sequence:3966 sequence: 3970 sequence:4003 sequence: 4004 sequence:4005 sequence: 4013 sequence:4063 sequence:4135 sequence:4136 sequence: 4137 sequence: 4148 sequence:4149 sequence: 4150 sequence: 4263 sequence: 4268 sequence:4277 sequence: 4287 sequence: 4292 sequence:4299 sequence: 4300 sequence:4388 sequence: 4414 sequence: 4420 sequence: 4548 sequence: 4553 sequence: 4562 sequence:4563 sequence: 4593 sequence: 4606 sequence:4613 sequence: 4620 sequence: 4636 sequence: 4639 sequence:4644 sequence: 4648 sequence: 4671 sequence: 4688 sequence:4689 sequence: 4694 sequence: 4782 sequence:4797 sequence: 4814 sequence:4870 sequence: 4872 sequence: 4875 sequence:4881 sequence:4891 sequence: 4913 sequence:4914 sequence: 4920 sequence: 4921 sequence: 4922 sequence:4930 sequence:4937 sequence:4941
Pol

Pol Pol Pol Pol Pol Pol Pol Pol Pol Pol Pol Pol Pol Pol Pol Pol Pol Pol Pol Pol Pol Pol Pol cPPT Pol cPPT Pol cPPT Pol cPPT Pol cPPT Pol cPPT

Pol Pol Pol Pol Pol Pol Pol Pol Vif Pol Vif Pol Vif Vif Vif Vif Vif Vif Vif Vif Vif Vif Vif Vif Vif Vif Vif Vif Vif Vif Vif Vif Vif Vif Vif Vif Vif Vif Vpr Vif $V p r$ Vif $V p r$ Vif Vpr Vif $V p r$ Vif Vpr Vif Vpr Vif Vpr 
ATGGACACTAGAGCTTTTAGAGG AAGCTGTTAGACACTTTCCTAGG TTAGACACTTTCCTAGGACATGG TTTCCTAGGACATGGCTCCACGG AATCCGTGGAGCCATGTCCTAGG AGGACATGGCTCCACGGATTAGG GGACATGGCTCCACGGATTAGGG AGATATATTGCCCTAATCCGTGG AATTATGGGGACACTTGGGCAGG TGGGGACACTTGGGCAGGAGTGG GGACACTTGGGCAGGAGTGGAGG GGGTGTCGACATAGCAGAATAGG CTACAAAGGAGAGCAAGAAATGG GGCTCTAGTCTAGGATCTACTGG TAGATCCTAGACTAGAGCCCTGG TGCTTCCAGGGCTCTAGTCTAGG CTAGAGCCCTGGAAGCATCCAGG TGACTTCCTGGATGCTTCCAGGG CTGACTTCCTGGATGCTTCCAGG TCCAGGAAGTCAGCCTAAGACGG GCCGTCTTAGGCTGACTTCCTGG CAAGTGTTACAAGCCGTCTTAGG AAAGCCTTAGGCATCTCCTATGG CCTTAGGCATCTCCTATGGCAGG TCTCCTATGGCAGGAAGAAGCGG TCTCCGCTTCTTCCTGCCATAGG ACAGCGACGAAGAGCTCCTCAGG TGATGAGTCTGACGGTCCTGAGG GAGAAGCTTGATGAGTCTGACGG ATAGAAAGAGCAGAAGACAGTGG GACAGTGGCAATGAGAGTGAAGG CAATGAGAGTGAAGGAGATCAGG GGAAGAATTGTCAGCACTTGTGG ATTGTCAGCACTTGTGGAGATGG TTGTCAGCACTTGTGGAGATGGG TGTCAGCACTTGTGGAGATGGGG AGATGGGGCATCTTGCTCCTTGG GATGGGGCATCTTGCTCCTTGGG GGGAGCAGCAGGAAGCACTATGG GGAGCAGCAGGAAGCACTATGGG CGCAGCGTCAGTGACGCTGACGG GTCAGTGACGCTGACGGTACAGG CAGGCCAGACTATTATTGTCTGG AACAGCAGAACAATCTGCTGAGG ACAGCAGAACAATCTGCTGAGGG CAATCTGCTGAGGGCTATTGAGG ATCTGTTGCAACTCACAGTCTGG TCTGTTGCAACTCACAGTCTGGG CTGTTGCAACTCACAGTCTGGGG CTGGGGCATCAAGCAGCTCCAGG CCAGGCAAGAGTCTTAGCTGTGG AGCTGTGGAAAGATACCTAAAGG TCGATTCCTCCGGGCCTGTCGGG TTCGATTCCTCCGGGCCTGTCGG CCCGGAGGAATCGAAGAAGAAGG ACCTTCTTCTTCGATTCCTCCGG GAGAGAGACAGAGACACATCCGG AGACACATCCGGACGCTTAGTGG ACATCCGGACGCTTAGTGGATGG GAATCCATCCACTAAGCGTCCGG CAATTTTCTGGGTCGATCTGCGG GGTGGTAGCTGAAGAGGCACAGG TCAAGCGGTGGTAGCTGAAGAGG AGAGTAAGTCTCTCAAGCGGTGG TCAAGAGTAAGTCTCTCAAGCGG CTTGATTGTAACGAGGATTGTGG AACGAGGATTGTGGAACTTCTGG ACGAGGATTGTGGAACTTCTGGG TTGTGGAACTTCTGGGACGCAGG TGTGGAACTTCTGGGACGCAGGG GTGGAACTTCTGGGACGCAGGGG sequence: 4960 sequence: 4995 sequence:5001 sequence:5009 sequence:5012 sequence:5015 sequence:5016 sequence:5026 sequence:5054 sequence:5059 sequence:5062 sequence:5132 sequence: 5162 sequence: 5187 sequence:5191 sequence:5196 sequence:5202 sequence:5208 sequence:5209 sequence:5219 sequence: 5220 sequence:5232 sequence:5301 sequence: 5305 sequence:5314 sequence:5317 sequence:5339 sequence: 5355 sequence: 5363 sequence: 5550 sequence: 5565 sequence: 5573 sequence:5594 sequence: 5600 sequence:5601 sequence:5602 sequence: 5617 sequence: 5618 sequence: 7121 sequence: 7122 sequence: 7145 sequence:7151 sequence: 7170 sequence:7201 sequence: 7202 sequence: 7211 sequence: 7243 sequence: 7244 sequence:7245 sequence:7262 sequence:7280 sequence: 7295 sequence:7731 sequence:7732 sequence: 7740 sequence:7741 sequence: 7767 sequence: 7778 sequence: 7782 sequence:7786 sequence: 7813 sequence:7838 sequence:7844 sequence:7856 sequence:7859 sequence:7877 sequence:7886 sequence:7887 sequence:7894 sequence:7895 sequence:7896

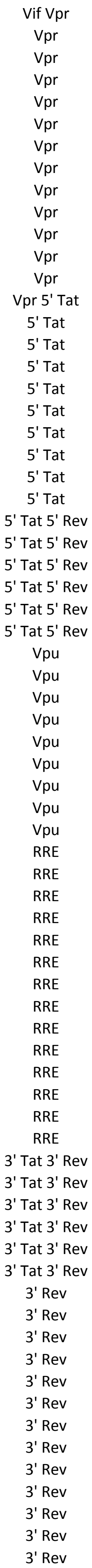


TGGAACTTCTGGGACGCAGGGGG ACTTCTGGGACGCAGGGGGTGGG GGTGGGAAGCCCTCAAATATTGG GGGAAGCCCTCAAATATTGGTGG GGAGATTCCACCAATATTTGAGG TCTCCTACAGTATTGGAGTCAGG GTTCCTGACTCCAATACTGTAGG AGCAAGTGGTCAAAAATGAGTGG AGTGGTCAAAAATGAGTGGGTGG TGAGTGGGTGGCCTGCTGTAAGG GAGTGGGTGGCCTGCTGTAAGGG CTCATTCTTTCCCTTACAGCAGG TGAGCCAGCAGCAGATGGAGTGG GAGCCAGCAGCAGATGGAGTGGG GCTCCCACTCCATCTGCTGCTGG GGGAGCAGCATCTAGAGACCTGG TCTAGAGACCTGGAAAAACATGG TGATTGCTCCATGTTTTTCCAGG CTACCAATGCTGATTGTGCCTGG TAGCCAGGCACAATCAGCATTGG TGCCTGGCTAGAAGCACAAGAGG CTCCTCTTGTGCTTCTAGCCAGG CTGGCTAGAAGCACAAGAGGAGG GCTAGAAGCACAAGAGGAGGAGG AAGGTACCTGAGGTTTGACTGGG ATTGGTCTTAAAGGTACCTGAGG AGAGCTCCCTTGTAAGTCATTGG AAAGAAAAGGGGGGACTGGAAGG AAGAAAAGGGGGGACTGGAAGGG GACAAGATATCCTTGATCTGTGG TATGGTAGACCCACAGATCAAGG CTGTGGGTCTACCATACACAAGG CAGGGAAGTAGCCTTGTGTATGG CACAAGGCTACTTCCCTGATTGG GTGTGTAATTCTGCCAATCAGGG GGTGTGTAATTCTGCCAATCAGG GATTGGCAGAATTACACACCAGG ATTGGCAGAATTACACACCAGGG CAGAATTACACACCAGGGCCAGG AGAATTACACACCAGGGCCAGGG GAATTACACACCAGGGCCAGGGG GGGAATCTGACCCCTGGCCCTGG GTCAGTGGGAATCTGACCCCTGG GTCAGATTCCCACTGACCTTTGG GATTCCCACTGACCTTTGGATGG AGCACCATCCAAAGGTCAGTGGG AAGCACCATCCAAAGGTCAGTGG TAGCTTGAAGCACCATCCAAAGG AGTACCAGTTGAGCCAGACAAGG TCTACCTTGTCTGGCTCAACTGG TGAGCCAGACAAGGTAGAAGAGG TTGGCCTCTTCTACCTTGTCTGG AAGGTAGAAGAGGCCAATGAAGG AGGTAGAAGAGGCCAATGAAGGG GGTAGAAGAGGCCAATGAAGGGG GCAGTTGTTTTCCCCTTCATTGG TTACACCCTATGAGCCAGCATGG TACACCCTATGAGCCAGCATGGG CCCTATGAGCCAGCATGGGATGG TCCATCCCATGCTGGCTCATAGG CCAGCATGGGATGGAAGACCCGG GTGGAAGTTTGACAGCCGCCTGG CCGCCTGGCATTGCATCACATGG GGGCCATGTGATGCAATGCCAGG CATGGCCCGAGAGAAGCATCCGG GTACTCCGGATGCTTCTCTCGGG AGTACTCCGGATGCTTCTCTCGG GAAGCATCCGGAGTACTACAAGG CAGCAGTCCTTGTAGTACTCCGG CTGACATCGAGCTTTCTACAAGG TGACATCGAGCTTTCTACAAGGG sequence:7897

sequence:7901

sequence:7918

sequence:7921

sequence: 7928

sequence:7946

sequence:7949

sequence: 8131

sequence: 8135

sequence: 8147

sequence: 8148

sequence: 8158

sequence: 8196

sequence: 8197

sequence: 8200

sequence: 8217

sequence: 8227

sequence: 8235

sequence: 8276

sequence: 8279

sequence: 8292

sequence: 8294

sequence: 8295

sequence: 8298

sequence: 8334

sequence:8344

sequence: 8362

sequence: 8404

sequence: 8405

sequence: 8447

sequence: 8457

sequence: 8464

sequence: 8475

sequence: 8480

sequence: 8493

sequence: 8494

sequence: 8497

sequence:8498

sequence: 8503

sequence: 8504

sequence: 8505

sequence: 8515

sequence: 8521

sequence: 8527

sequence:8531

sequence: 8535

sequence: 8536

sequence: 8543

sequence: 8565

sequence: 8569

sequence: 8574

sequence: 8578

sequence:8584

sequence: 8585

sequence: 8586

sequence:8597

sequence: 8623

sequence: 8624

sequence: 8628

sequence: 8629

sequence: 8637

sequence: 8676

sequence:8691

sequence:8694

sequence:8709

sequence:8714

sequence:8715

sequence:8721

sequence: 8728

sequence: 8748

sequence: 8749

\section{3' Rev \\ 3' Rev \\ 3' Rev \\ 3' Rev \\ 3' Rev \\ 3' Rev \\ 3' Rev \\ Nef \\ Nef \\ Nef \\ Nef \\ Nef \\ Nef \\ Nef \\ Nef \\ Nef \\ Nef \\ Nef \\ Nef \\ Nef \\ Nef \\ Nef \\ Nef \\ Nef \\ Nef \\ Nef \\ Nef \\ Nef \\ Nef}

Nef LTR U3

Nef LTR U3

Nef LTR U3

Nef LTR U3

Nef LTR U3

Nef LTR U3

Nef LTR U3

Nef LTR U3

Nef LTR U3

Nef LTR U3

Nef LTR U3

Nef LTR U3

Nef LTR U3

Nef LTR U3

Nef LTR U3

Nef LTR U3

Nef LTR U3

Nef LTR U3

Nef LTR U3

Nef LTR U3

Nef LTR U3

Nef LTR U3

Nef LTR U3

Nef LTR U3

Nef LTR U3

Nef LTR U3

Nef LTR U3

Nef LTR U3

Nef LTR U3

Nef LTR U3

Nef LTR U3

Nef LTR U3

Nef LTR U3

Nef LTR U3

Nef LTR U3

Nef LTR U3

Nef LTR U3

Nef LTR U3

Nef LTR U3

Nef LTR U3

LTR U3

LTR U3 
TTCTACAAGGGACTTTCCGCTGG TCTACAAGGGACTTTCCGCTGGG CTACAAGGGACTTTCCGCTGGGG CTTTCCGCTGGGGACTTTCCAGG TTTCCGCTGGGGACTTTCCAGGG CCGCTGGGGACTTTCCAGGGAGG GGGGACTTTCCAGGGAGGCGTGG CTTTCCAGGGAGGCGTGGCCTGG TTTCCAGGGAGGCGTGGCCTGGG TGCTTATATGCAGCATCTGAGGG CTGCTTATATGCAGCATCTGAGG CAGCTGCTTTTTGCCTGTACTGG AGCTGCTTTTTGGCTGTACTGGG TTGCCTGTACTGGGTCTCTCTGG TAACCAGAGAGACCCAGTACAGG GGTTAGACCAGATCAGAGCCTGG GTTAGACCAGATCAGAGCCTGGG AGAGCTCCCAGGCTCTGATCTGG ATCAGAGCCTGGGAGCTCTCTGG TAGTTAGCCAGAGAGCTCCCAGG TGGGAGCTCTCTGGCTAACTAGG GGGAGCTCTCTGGCTAACTAGGG CTTTATTGAGGCTTAAGCAGTGG ACTCAAGGCAAGCTTTATTGAGG CACACTACTTGAAGCACTCAAGG sequence:8761

sequence: 8762

sequence: 8763

sequence: 8773

sequence: 8774

sequence: 8777

sequence: 8782

sequence: 8787

sequence: 8788

sequence:8831

sequence: 8832

sequence: 8852

sequence: 8853

sequence: 8862

sequence: 8865

sequence: 8883

sequence: 8884

sequence: 8890

sequence:8894

sequence:8901

sequence: 8903

sequence:8904

sequence:8930

sequence: 8942

sequence: 8957 
HIV 671

\begin{tabular}{|c|c|c|c|c|}
\hline HIV-1 671 Target sequence & 5' end of gRNA target site & Strand & \% GC content & HIV-1 target \\
\hline CAGCTAGCCAGAGAGCTCCCAGG & sequence: 4 & - & 65 & LTR R \\
\hline CTGGGAGCTCTCTGGCTAGCTGG & sequence:5 & + & 65 & LTR R \\
\hline TGGGAGCTCTCTGGCTAGCTGGG & sequence: 6 & + & 60 & LTR R \\
\hline GGGAGCTCTCTGGCTAGCTGGGG & sequence:7 & + & 65 & LTR R \\
\hline CTTTATTGAGGCTTAAGCAGTGG & sequence:33 & - & 40 & LTR R \\
\hline ACTCAAGGCAAGCTTTATTGAGG & sequence: 45 & - & 40 & LTR R \\
\hline CACACTACTTAAAGCACTCAAGG & sequence:60 & - & 40 & LTR U5 \\
\hline GCCCGTCTGTTATGTGACTCTGG & sequence: 84 & + & 55 & LTR U5 \\
\hline ACCAGAGTCACATAACAGACGGG & sequence: 85 & - & 45 & LTR U5 \\
\hline TACCAGAGTCACATAACAGACGG & sequence: 86 & - & 40 & LTR U5 \\
\hline СACTGACTAAAAGGGTCTGAGGG & sequence:118 & - & 45 & LTR U5 \\
\hline ACACTGACTAAAAGGGTCTGAGG & sequence:119 & - & 45 & LTR U5 \\
\hline TCAGACCCTTTTAGTCAGTGTGG & sequence:121 & + & 45 & LTR U5 \\
\hline GTGTGGAAAATCTCTAGCAGTGG & sequence:138 & + & 45 & LTR U5 \\
\hline TCTAGCAGTGGCGCCCGAACAGG & sequence:150 & + & 65 & LTR U5 \\
\hline CTAGCAGTGGCGCCCGAACAGGG & sequence:151 & + & 65 & LTR U5 \\
\hline CTTTCTTCAAGTCCCTGTTCGGG & sequence:163 & - & 45 & \\
\hline ACTTTCTTCAAGTCCCTGTTCGG & sequence:164 & - & 40 & \\
\hline CTGCGTCGAGAGATCTCCTCTGG & sequence:191 & - & 60 & \\
\hline CAGAGGAGATCTCTCGACGCAGG & sequence:192 & + & 60 & \\
\hline AGATCTCTCGACGCAGGACTCGG & sequence:198 & + & 55 & \\
\hline TGCTGAAGAAGAAGCGCGCGCGG & sequence:223 & + & 60 & \\
\hline GAAGAAGCGCGCGCGGCAAGAGG & sequence:230 & + & 70 & \\
\hline TTTGACTAGCGGAGGCTAGAAGG & sequence:287 & + & 50 & \\
\hline GAGGCTAGAAGGAGAGAGATGGG & sequence:298 & + & 50 & \\
\hline GCGAGAGCGTCAGTATTAAGCGG & sequence:322 & + & 50 & Gag \\
\hline CGAGAGCGTCAGTATTAAGCGGG & sequence:323 & + & 50 & Gag \\
\hline GAGAGCGTCAGTATTAAGCGGGG & sequence:324 & + & 50 & Gag \\
\hline AGAGCGTCAGTATTAAGCGGGGG & sequence:325 & + & 50 & Gag \\
\hline GCGGGGGAGAATTAGATAGATGG & sequence:341 & + & 50 & Gag \\
\hline CGGGGGAGAATTAGATAGATGGG & sequence:342 & + & 45 & Gag \\
\hline GAAAAAATTCGGTTAAGGCCAGG & sequence:364 & + & 40 & Gag \\
\hline AAAATTCGGTTAAGGCCAGGGGG & sequence:367 & + & 45 & Gag \\
\hline GAACGATTCGCAGTTAATCCTGG & sequence:439 & + & 45 & Gag \\
\hline GGCCTTTTAGAGACATCAGAAGG & sequence:460 & + & 45 & Gag \\
\hline AGCCTTCTGATGTCTCTAAAAGG & sequence:462 & - & 40 & Gag \\
\hline AGAAGGCTGTAGACAAATACTGG & sequence:477 & + & 40 & Gag \\
\hline GAAGGCTGTAGACAAATACTGGG & sequence:478 & + & 40 & Gag \\
\hline CTACAACCAGCCCTTCAGACAGG & sequence:505 & + & 55 & Gag \\
\hline TCTGATCCTGTCTGAAGGGCTGG & sequence:511 & - & 55 & Gag \\
\hline TTCTTCTGATCCTGTCTGAAGGG & sequence:515 & - & 40 & Gag \\
\hline GTTCTTCTGATCCTGTCTGAAGG & sequence:516 & - & 45 & Gag \\
\hline сCCTCTATTGTGTGCATGCAAGG & sequence:566 & + & 50 & Gag \\
\hline TCCTTGCATGCACACAATAGAGG & sequence:567 & - & 45 & Gag \\
\hline TTGTGTGCATGCAAGGATAGAGG & sequence:573 & + & 45 & Gag \\
\hline GATAGAGGTAAAAGACACCAAGG & sequence:588 & + & 40 & Gag \\
\hline CAGCAAGCAGAAGCTGACGCAGG & sequence:670 & + & 60 & Gag \\
\hline TGACGCAGGAAAAAACAACCCGG & sequence:684 & + & 45 & Gag \\
\hline TAGGGTAATTCTGGCTGACCGGG & sequence:702 & - & 50 & Gag \\
\hline ATAGGGTAATTCTGGCTGACCGG & sequence:703 & - & 45 & Gag \\
\hline TCTGCACTATAGGGTAATTCTGG & sequence:711 & - & 40 & Gag \\
\hline CTTGGAGATTCTGCACTATAGGG & sequence:720 & - & 40 & Gag \\
\hline CCTATAGTGCAGAATCTCCAAGG & sequence:721 & + & 45 & Gag \\
\hline CTATAGTGCAGAATCTCCAAGGG & sequence:722 & + & 40 & Gag \\
\hline GCAGAATCTCCAAGGGCAAATGG & sequence:729 & + & 50 & Gag \\
\hline CCAAGGGCAAATGGTACATCAGG & sequence:738 & + & 50 & Gag \\
\hline GGGTATTACTTCTGGGCTGAAGG & sequence:812 & - & 50 & Gag \\
\hline GGTGTTTAAATCTTGTGGGGTGG & sequence:860 & - & 45 & Gag \\
\hline AAACACCATGCTAAACACAGTGG & sequence:876 & + & 40 & Gag \\
\hline AACACCATGCTAAACACAGTGGG & sequence:877 & + & 40 & Gag \\
\hline ACACCATGCTAAACACAGTGGGG & sequence:878 & + & 45 & Gag \\
\hline CACCATGCTAAACACAGTGGGGG & sequence:879 & + & 50 & Gag \\
\hline ACCATGCTAAACACAGTGGGGGG & sequence:880 & + & 50 & Gag \\
\hline TCCCCCCACTGTGTTTAGCATGG & sequence:881 & - & 55 & Gag \\
\hline TTCTGCAGCTTCCTCATTGATGG & sequence:935 & - & 45 & Gag \\
\hline TCAATGAGGAAGCTGCAGAATGG & sequence:938 & + & 45 & Gag \\
\hline CAATGAGGAAGCTGCAGAATGGG & sequence:939 & + & 45 & Gag \\
\hline AGATTGCATCCAGTGCATGCCGG & sequence:964 & + & 50 & Gag \\
\hline GATTGCATCCAGTGCATGCCGGG & sequence:965 & + & 55 & Gag \\
\hline GCAATAGGCCCGGCATGCACTGG & sequence:973 & - & 65 & Gag \\
\hline
\end{tabular}


CATGCCGGGCCTATTGCACCAGG CTGGCCTGGTGCAATAGGCCCGG CTCATCTGGCCTGGTGCAATAGG CTTGGTTCTCTCATCTGGCCTGG CAGGCCAGATGAGAGAACCAAGG AGGCCAGATGAGAGAACCAAGGG GGCCAGATGAGAGAACCAAGGGG TTCCCCTTGGTTCTCTCATCTGG CCAAGGGGAAGTGACATAGCAGG AGGAACTACTAGTACCCTTCAGG AGTACCCTTCAGGAACAAATAGG CCCTTCAGGAACAAATAGGATGG TCCATCCTATTTGTTCCTGAAGG AATAATCCACCTATCCCAGTAGG ATTTCTCCTACTGGGATAGGTGG GTATAGCCCTACCAGCATTCTGG TTATGTCCAGAATGCTGGTAGGG TTTATGTCCAGAATGCTGGTAGG GGACATAAAACAAGGACCAAAGG TAGTCTCTAAAGGGTTCCTTTGG AAGAGCTGAGCAAGCCTCACAGG TTGGATGACAGAAACCTTGTTGG GTTCGAATTTTGGACCAACAAGG ATTTCTTCTAGTGTAGCTGCTGG AGAAATGATGACCGCATGTCAGG GAAATGATGACCGCATGTCAGGG ATGACCGCATGTCAGGGAGTAGG TGACCGCATGTCAGGGAGTAGGG GACCGCATGTCAGGGAGTAGGGG ACCGCATGTCAGGGAGTAGGGGG TCCCCCTACTCCCTGACATGCGG TGTCAGGGAGTAGGGGGACCCGG AAAACTCTCGCTTTATGGCCGGG CAAAACTCTCGCTTTATGGCCGG CGGCCATAAAGCGAGAGTTTTGG CAGCCAAAACTCTCGCTTTATGG TAAAGCGAGAGTTTTGGCTGAGG TGGCAGGTGCACTTGTTACTTGG CCTGCCATAATGATGCAGAGAGG ATTGCCTCTCTGCATCATTATGG GGCACATAGCCAGAAATTGCAGG GCACATAGCCAGAAATTGCAGGG TCTAGGGGCCCTGCAATTTCTGG ATTGCAGGGCCCCTAGAAAAAGG TTGCAGGGCCCCTAGAAAAAGGG GCCCCTAGAAAAAGGGCTGTTGG TCCAACAGCCCTTTTTCTAGGGG TTCCAACAGCCCTTTTTTCTAGGG TTTCCAACAGCCCTTTTTCTAGG GCTGTTGGAAATGTGAAAGAAGG GAAGATTTGGCCTTCCCACAAGG AAGATTTGGCCTTCCCACAAGGG AGATTTGGCCTTCCCACAAGGGG TTTGGCCTTCCCACAAGGGGAGG CCTTCCCACAAGGGGAGGCCAGG CTTCCCACAAGGGGAGGCCAGGG CTGCTCTGAAGAAAATTCCCTGG TGAGAAGGGGCTGTTGGCTCTGG CTGCTCTGAGAAGGGGCTGTTGG CTCTGGTCTGCTCTGAGAAGGGG GCTCTGGTCTGCTCTGAGAAGGG GGCTCTGGTCTGCTCTGAGAAGG TCTGGTGGGGCTGTTGGCTCTGG CTCTCTTCTGGTGGGGCTGTTGG CCCCACCAGAAGAGAGCTTCAGG ACCTGAAGCTCTCTTCTGGTGGG AACCTGAAGCTCTCTTCTGGTGG CCAGAAGAGAGCTTCAGGTTTGG CAGAAGAGAGCTTCAGGTTTGGG AGAAGAGAGCTTCAGGTTTGGGG AGACAACAACTCCCTCTCAGAGG sequence:979

sequence:983

sequence:988

sequence:997

sequence:998

sequence:999

sequence: 1000

sequence:1002

sequence:1015

sequence:1035

sequence:1045

sequence: 1049

sequence: 1050

sequence:1078

sequence: 1084

sequence: 1152

sequence:1158

sequence:1159

sequence:1173

sequence:1189

sequence:1236

sequence:1269

sequence: 1283

sequence:1339

sequence:1356

sequence:1357

sequence:1363

sequence:1364

sequence:1365

sequence:1366

sequence:1367

sequence:1372

sequence: 1390

sequence:1391

sequence:1392

sequence:1395

sequence:1398

sequence: 1428

sequence:1444

sequence: 1448

sequence: 1520

sequence:1521

sequence:1529

sequence:1535

sequence:1536

sequence:1543

sequence:1544

sequence: 1545

sequence:1546

sequence: 1558

sequence:1624

sequence: 1625

sequence:1626

sequence: 1629

sequence:1634

sequence:1635

sequence: 1652

sequence:1676

sequence:1682

sequence: 1689

sequence: 1690

sequence:1691

sequence:1706

sequence:1712

sequence:1719

sequence: 1720

sequence:1721

sequence:1724

sequence: 1725

sequence:1726

sequence: 1752
65

65 
AACAACTCCCTCTCAGAGGCAGG GTTGGCTCCTGCCTCTGAGAGGG TGTTGGCTCCTGCCTCTGAGAGG GAGGCAGGAGCCAACAGACAAGG GGATACAGTTCCTTGTCTGTTGG AGTGATTTGAGGGAAGCTAAAGG GCTTCCCTCAAATCACTCTTTGG GCTGCCAAAGAGTGATTTGAGGG AAAGATAGGGGGGCAGCTAAAGG AAGGAAGCTCTATTAGACACAGG AAATGGATTTGCCAGGAAGATGG CCAAAAATGATAGGGGGAATTGG AAAATGATAGGGGGAATTGGAGG CCTACACCTGTCAACATAATTGG AGAAATCTGTTGACTCAGCTTGG ACTTTTGGGCCATCCATTCCTGG ATGGCCCAAAAGTCAAACAATGG TGTACAGAACTGGAAAAGGAAGG GACTTCTGGGAAGTTCAATTAGG ATTAGGAATACCACACCCTGCGG TTAGGAATACCACACCCTGCGGG TAGGAATACCACACCCTGCGGGG TTTTTTAACCCCGCAGGGTGTGG ATCAGTAACAGTACTGGACGTGG TCAGTAACAGTACTGGACGTGGG GGTGTCTCATTGTTTGTACTAGG AGTACAAACAATGAGACACCAGG GTACAAACAATGAGACACCAGGG TCAGTATAATGTGCTTCCACAGG CAGTATAATGTGCTTCCACAGGG ATAATGTGCTTCCACAGGGATGG GTGCTTCCACAGGGATGGAAAGG GGTGATCCTTTCCATCCCTGTGG AGAAAGAACCCCCATTCCTTTGG AGAACCCCCATTCCTTTGGATGG GAACCCCCATTCCTTTGGATGGG TAACCCATCCAAAGGAATGGGGG ATAACCCATCCAAAGGAATGGGG CATAACCCATCCAAAGGAATGGG TCATAACCCATCCAAAGGAATGG GGAGTTCATAACCCATCCAAAGG GTACTGTCCATTTATCAGGATGG GGCTGTACTGTCCATTTATCAGG TCTTTTTCTGGCAGCACTATAGG GTGCTGCCAGAAAAAGACCCTGG TGACAGCCAGGGTCTTTTTTCTGG CTGTATGTCATTGACAGCCAGGG TCTGTATGTCATTGACAGCCAGG TGGGCAAGTCAGATTTATCCAGG GGGCAAGTCAGATTTATCCAGGG TTAGTGCTTTAGTTCCCCTAAGG AGCTCTGCTTCTGCTGTTAGTGG GAAATACAGAAGCAGGGACAAGG AGAAGCAGGGACAAGGCCAATGG CAGGAAAGTATGCAAAAGTGAGG GGAAAGTATGCAAAAGTGAGGGG AAGAAACATGGGAAGCATGGTGG AAGCATGGTGGACAGAGTATTGG CAGAGTATTGGCAAGCCACCTGG AAGCCACCTGGATTCCTGAGTGG AGCCACCTGGATTCCTGAGTGGG AAACTCCCACTCAGGAATCCAGG GTATTGACAAACTCCCACTCAGG CCCCTCCCTTAGTGAAATTATGG CTATGGGTTCCTTTTCTAACTGG GAAAGTTTCTGCTCCTACTATGG ATGTAGATGGGGCAGCTAATAGG TGTAGATGGGGCAGCTAATAGGG GCAGGATATGTTACTGACAGAGG CAGGATATGTTACTGACAGAGGG CATCTAGCTTTGCAAGATTCAGG sequence: 1756

sequence: 1763

sequence: 1764

sequence: 1771

sequence: 1781

sequence: 1802

sequence: 1808

sequence: 1812

sequence: 1851

sequence: 1870

sequence:1916

sequence: 1942

sequence: 1945

sequence: 2047

sequence: 2071

sequence: 2149

sequence:2159

sequence: 2221

sequence: 2365

sequence: 2382

sequence: 2383

sequence: 2384

sequence: 2392

sequence: 2421

sequence: 2422

sequence:2506

sequence: 2509

sequence: 2510

sequence: 2541

sequence: 2542

sequence: 2546

sequence: 2551

sequence: 2557

sequence: 2774

sequence: 2778

sequence: 2779

sequence: 2782

sequence: 2783

sequence: 2784

sequence: 2785

sequence: 2790

sequence:2811

sequence: 2815

sequence: 2836

sequence: 2842

sequence: 2848

sequence: 2859

sequence: 2860

sequence: 2902

sequence: 2903

sequence:2952

sequence: 2986

sequence: 3088

sequence:3095

sequence:3158

sequence: 3160

sequence: 3290

sequence: 3302

sequence:3314

sequence:3326

sequence: 3327

sequence:3332

sequence: 3340

sequence: 3362

sequence: 3387

sequence: 3404

sequence: 3428

sequence: 3429

sequence: 3469

sequence: 3470

sequence:3553
Gag Pol

Gag Pol

Gag Pol

Gag Pol

Gag Pol

Gag Pol

Gag Pol

Gag Pol

$\mathrm{Pol}$

Pol

Pol

Pol

Pol

Pol

Pol

Pol

Pol

Pol

Pol

Pol

Pol

Pol

Pol

Pol

Pol

Pol

Pol

Pol

Pol

Pol

Pol

Pol

Pol

Pol

Pol

Pol

Pol

Pol

Pol

Pol

Pol

Pol

Pol

Pol

Pol

Pol

Pol

Pol

Pol

Pol

Pol

Pol

Pol

Pol

Pol

Pol

Pol

Pol

Pol

Pol

Pol

Pol

Pol

Pol

Pol

Pol

Pol

Pol

Pol

Pol

Pol 
AGGAAAAGGTCTACCTGGCATGG GGAAAAGGTCTACCTGGCATGGG TGTGTGCTGGCACCCATGCCAGG GCATGGGTGCCAGCACACAAAGG CCAGCACACAAAGGAATTGGAGG TCACAGTAATTGGAGAGCAATGG CTTTTGCTACTACAGGTGGCAGG CTAAAAGGAGAAGCCATGCATGG GGACAAGTAGACTGTAGTCCAGG TAGACTGTAGTCCAGGAATATGG CAATCTAGCTGCCATATTCCTGG TGGCTACATGAACTGCTACCAGG GTAGCAGTTCATGTAGCCAGTGG GAAGTTATTCCAGCAGAAACAGG GGGGCCATTGTCTGTATGTATGG CTGCAGTACTGATGAAATTGGGG CTGCAGTTAAGGCCGCCTGTTGG CAGTTAAGGCCGCCTGTTGGTGG AGTTAAGGCCGCCTGTTGGTGGG TAAGGCCGCCTGTTGGTGGGCGG AAGGCCGCCTGTTGGTGGGCGGG GATCCCCGCCCACCAACAGGCGG CTTGATCCCCGCCCACCAACAGG TTGGTGGGCGGGGATCAAGCAGG CCCTACAATCCCCAAAGTCAAGG TCCTTGACTTTGGGGATTGTAGG TCTACTACTCCTTGACTTTGGGG TATAGGACAGGTAAGAGAGCAGG TCTCAAGACAGCAGTACAAATGG TAAAAGAAAAGGGGGGATTGGGG GGGATTGGGGAGTATAGTGCAGG GGATTGGGGAGTATAGTGCAGGG GATTGGGGAGTATAGTGCAGGGG GGGACAGCAGAGATCCACTTTGG AGCAGAGATCCACTTTGGAAAGG TTTGCTGGTCCTTTCCAAAGTGG AAGGACCAGCAAAGCTTCTCTGG CCAGCAAAGCTTCTCTGGAAAGG AAGCTTCTCTGGAAAGGTGAAGG AGCTTCTCTGGAAAGGTGAAGGG GATTATGGAAAACAGATGGCAGG TGATTGTGTGGCAAGTAGACAGG TGTGGCAAGTAGACAGGATGAGG ACAGGATGAGGATTAGAACATGG CTTTCCCTGACCTATACATATGG GGTCAGGGAAAGCTAAAAAATGG TCAGAAGTACACATCCCACTAGG CAGAAGTACACATCCCACTAGGG AGAAGTACACATCCCACTAGGGG AGTACACATCCCACTAGGGGAGG CTAATTTAGCCTCCCCTAGTGGG ACTAATTTAGCCTCCCCTAGTGG ACATATTGGGGTCTGCATACAGG TGCATACAGGAGAAAGAGACTGG GTCAGGGAGTCTCCATAGAATGG AGGGAGTCTCCATAGAATGGAGG GGGAGTCTCCATAGAATGGAGGG TCTCCATAGAATGGAGGGAAAGG TCTCCTTTCCCTCCATTCTATGG TAGGTCAGGGTCTACTTGTGTGG TTAGTTGGTCTGCTAGGTCAGGG ATTAGTTGGTCTGCTAGGTCAGG CATGAATTAGTTGGTCTGCTAGG TAGGACGTTTAGTTAGCCCTAGG CTGCTTGATATTCACACCTAGGG CCTAGGTGTGAATATCAAGCAGG CAAGCAGGACATAACAAGGTAGG AAGCAGGACATAACAAGGTAGGG GGTAGGGTCTCTACAGTACTTGG AGCCACCTTTGCCTAGTGTTAGG TTCCTAACACTAGGCAAAGGTGG sequence: 3689

sequence: 3690

sequence:3702

sequence:3706

sequence:3715

sequence:3831

sequence:3867

sequence:3919

sequence: 3940

sequence: 3947

sequence:3958

sequence:4005

sequence:4009

sequence:4045

sequence: 4121

sequence:4140

sequence: 4157

sequence: 4160

sequence:4161

sequence:4164

sequence: 4165

sequence:4169

sequence:4172

sequence: 4176

sequence:4210

sequence:4211

sequence: 4219

sequence:4269

sequence:4299

sequence:4341

sequence: 4354

sequence: 4355

sequence:4356

sequence:4469

sequence: 4474

sequence:4483

sequence: 4493

sequence: 4498

sequence: 4504

sequence: 4505

sequence: 4594

sequence: 4620

sequence:4626

sequence: 4638

sequence: 4678

sequence: 4689

sequence: 4754

sequence: 4755

sequence: 4756

sequence: 4759

sequence: 4768

sequence:4769

sequence:4799

sequence: 4812

sequence: 4842

sequence: 4845

sequence:4846

sequence:4851

sequence: 4854

sequence:4881

sequence:4894

sequence: 4895

sequence:4900

sequence:4971

sequence:4987

sequence: 4988

sequence:5003

sequence:5004

sequence: 5020

sequence:5076

sequence:5078

50

50 
AGTTTCCTAACACTAGGCAAAGG TCTGTCAGTTTCCTAACACTAGG GGAAACTGACAGAGGATAGATGG AGGATAGATGGAACAAGCCCCGG GAACAAGCCCCGGAAGACCAAGG AACAAGCCCCGGAAGACCAAGGG CTGTGGCCCTTGGTCTTCCGGGG TCTGTGGCCCTTGGTCTTCCGGG CTCTGTGGCCCTTGGTCTTCCGG ATGGCTCTCTCTGTGGCCCTTGG GCATTGTATGGCTCTCTCTGTGG AGAGAGAGCCATACAATGCATGG TCTAGTGTCCATGCATTGTATGG ATGGACACTAGAGCTTCTAGAGG GCTGTTAGACATTTTCCTAGGGG TTAGACATTTTCCTAGGGGATGG TTTCCTAGGGGATGGCTCCATGG AAGCCATGGAGCCATCCCCTAGG AGATATGTTGTCCTAAGCCATGG ACTTATGGGGATACTTGGGCAGG TGGGGATACTTGGGCAGGAGTGG GGATGTCAACATAGCAGAATAGG AGAATAGGCATTACTCGACGAGG GAATAGGCATTACTCGACGAGGG TTACTCGACGAGGGAGAGTAAGG CGACGAGGGAGAGTAAGGAATGG GGCTCTAGTCTAGGGTCTACTGG TAGACCCTAGACTAGAGCCCTGG GCTTCCAGGGCTCTAGTCTAGGG TGCTTCCAGGGCTCTAGTCTAGG CTAGAGCCCTGGAAGCATCCAGG TGACTTCCTGGATGCTTCCAGGG CTGACTTCCTGGATGCTTCCAGG GCAGTCTTAGGCTGACTTCCTGG AAAGGCTTAGGCATCTCCTATGG GCTTAGGCATCTCCTATGGCAGG TCTCCTATGGCAGGAAGAAGCGG TCTCCGCTTCTTCCTGCCATAGG ACAGCGACGAAGAACTCCTCAGG TGATGAGTCTGACTGTCCTGAGG AGAGAAAGAGCAGAAGACAGTGG CAGTGGCAATGAAAGTGAAGGGG CAATGAAAGTGAAGGGGATCAGG GGAAGAATTATCAGCTCTTGTGG AGATGGGGCATCATGCTCCTTGG GATGGGGCATCATGCTCCTTGGG TACAGATCATCAACGTCCCAAGG GGGAGCAGCAGGAAGCACTATGG GGAGCAGCAGGAAGCACTATGGG CACTATGGGCGCAGCGTCAATGG CGCAGCGTCAATGGCGCTGACGG GTCAATGGCGCTGACGGTACAGG CAGGCCAGACAATTATTGTCTGG CAATTTGCTGAGGGCTATTGAGG ATCTGCTGCAACTCACAGTCTGG TCTGCTGCAACTCACAGTCTGGG CTGCTGCAACTCACAGTCTGGGG CTGGGGCATTAAGCAGCTCCAGG GCAGCTCCAGGCAAGAGTCCTGG CTATAGCCAGGACTCTTGCCTGG GTAGGTATCTTTCTATAGCCAGG GGCTATAGAAAGATACCTACAGG TGTCGGGTCCCCTCGGAGCTGGG TCGATTCCTTCGGGCCTGTCGGG TTCGATTCCTTCGGGCCTGTCGG CCCGAAGGAATCGAAGAAGAAGG ACCTTCTTCTTCGATTCCTTCGG GAAGGAATCGAAGAAGAAGGTGG GAGAGAGACAGAGACGGATCCGG AGAGAGACAGAGACGGATCCGGG GGATCCGGGCCATTAGTGAATGG sequence: 5081

sequence:5087

sequence: 5097

sequence:5109

sequence:5119

sequence: 5120

sequence:5126

sequence:5127

sequence:5128

sequence: 5136

sequence:5143

sequence: 5147

sequence:5155

sequence:5166

sequence: 5203

sequence:5207

sequence:5215

sequence: 5218

sequence:5232

sequence:5260

sequence:5265

sequence: 5338

sequence:5353

sequence:5354

sequence:5363

sequence: 5368

sequence:5393

sequence: 5397

sequence:5401

sequence:5402

sequence: 5408

sequence:5414

sequence: 5415

sequence:5426

sequence:5507

sequence:5511

sequence: 5520

sequence:5523

sequence:5545

sequence: 5561

sequence:5756

sequence: 5773

sequence:5779

sequence: 5800

sequence:5823

sequence: 5824

sequence: 5840

sequence:7346

sequence:7347

sequence:7361

sequence:7370

sequence:7376

sequence:7395

sequence:7436

sequence:7468

sequence:7469

sequence: 7470

sequence:7487

sequence:7499

sequence:7505

sequence: 7517

sequence: 7520

sequence:7940

sequence:7956

sequence: 7957

sequence:7965

sequence:7966

sequence: 7968

sequence:7992

sequence:7993

sequence:8007
40

40

45

50

60

55

65

60

60

60

50

45

40

45

40

40

55

60

40

50

60

40

45

45

50

55

55

55

55

55

60

50

55

55

45

55

50

55

55

50

45

45

45

40

55

55

45

60

55

60

65

60

45

45

50

50

55

60

65

55

40

40

70

60

55

50

40

45

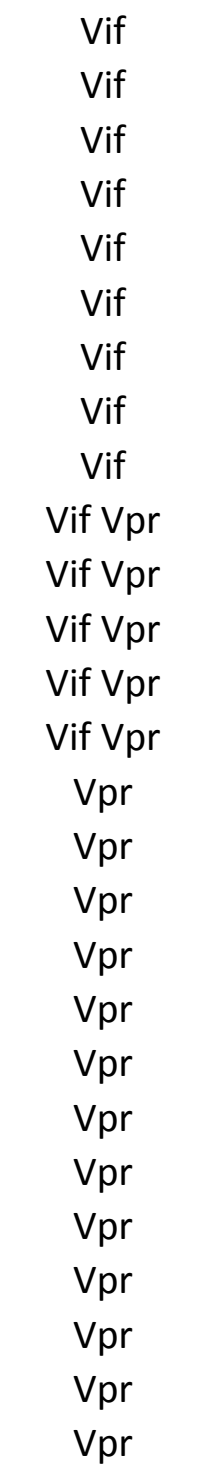

Vpr 5' Tat

Vpr 5' Tat

Vpr 5' Tat

Vpr 5' Tat

Vpr 5' Tat

5 ' Tat

5 ' Tat

5' Tat

5' Tat

5' Tat

5 ' Tat

5' Tat 5' Rev

5' Tat 5' Rev

$\mathrm{Vpu}$

Vpu

Vpu

Vpu

Vpu

Vpu

Vpu

RRE

RRE

RRE

RRE

RRE

RRE

RRE

RRE

RRE

RRE

RRE

RRE

RRE

RRE

RRE

3' Tat 3' Rev

3' Tat 3' Rev

3' Tat 3' Rev

3' Tat 3' Rev

3' Tat 3' Rev

3' Tat 3' Rev

3' Tat 3' Rev

3' Tat 3' Rev

3' Tat 3' Rev 
GAATCCATTCACTAATGGCCCGG CAATCATCTGGGTCGACCTGCGG TGAAGAGGCACAGGCTCCGCAGG GGTGGTAGCTGAAGAGGCACAGG TCAAGCGGTGGTAGCTGAAGAGG AGAGTAAGTCTCTCAAGCGGTGG TCAAGAGTAAGTCTCTCAAGCGG CTTGATTGTAGTGAGGATTGTGG AGTGAGGATTGTGGAACTTCTGG GTGAGGATTGTGGAACTTCTGGG TTGTGGAACTTCTGGGACGCAGG TGTGGAACTTCTGGGACGCAGGG GTGGAACTTCTGGGACGCAGGGG TGGAACTTCTGGGACGCAGGGGG ACTTCTGGGACGCAGGGGGTGGG GGTGGGAAGCCCTCAAATACTGG GGGAAGCCCTCAAATACTGGTGG GAGATTCCACCAGTATTTGAGGG GGAGATTCCACCAGTATTTGAGG GGTGGAATCTCCTGCAATATTGG TCTCCTGCAATATTGGATTCAGG GTTCCTGAATCCAATATTGCAGG AAGTGGTCAAAACATAAGGGGGG TGGTCAAAACATAAGGGGGGTGG CAAAACATAAGGGGGGTGGATGG TAAGGGGGGTGGATGGCCTGCGG GGGTGGATGGCCTGCGGTGAGGG TGCATTCTTTTCCCTCACCGCAGG CGAGCTGAGCCAGCAGCAGACGG GAGCTGAGCCAGCAGCAGACGGG AGCTGAGCCAGCAGCAGACGGGG TGAGCCAGCAGCAGACGGGGTGG GAGCCAGCAGCAGACGGGGTGGG GGGACCAGTATCTCGAGACTTGG TTTTCCAAGTCTCGAGATACTGG TCTCGAGACTTGGAAAAACATGG CTAATAATGCTGCTTGTGTCTGG TGTCTGGCTAGAAGCACAAGAGG CTGGCTAGAAGCACAAGAGGAGG GCTAGAAGCACAAGAGGAGGAGG GGGTTTTCCAGTCAGACCTCAGG AAAGGTACCTGAGGTCTGACTGG ATTGGTCTTAAAGGTACCTGAGG AAAGCTCCCTTGTAAGTCATTGG AATGACTTACAAGGGAGCTTTGG AAAGAAAAGGGGGGACTGGAAGG AAGAAAAGGGGGGACTGGAAGGG GACAAGATATCCTTGATCTGTGG TGTGGTAGACCCACAGATCAAGG CTGTGGGTCTACCACACACAAGG CAGGGAAGTAGCCTTGTGTGTGG CACAAGGCTACTTCCCTGATTGG ACAAGGCTACTTCCCTGATTGGG GTGTGTAGTTATCCCAATCAGGG GGTGTGTAGTTATCCCAATCAGG GATTGGGATAACTACACACCAGG ATTGGGATAACTACACACCAGGG GATAACTACACACCAGGGCCAGG GGATATCTGATCCCTGGCCCTGG ATCAGTGGATATCTGATCCCTGG GATATCCACTGATCTTTGGATGG AAGCACCATCCAAAGATCAGTGG AGTACCAGTTGATCCAGACCAGG TCTACCTGGTCTGGATCAACTGG TGATCCAGACCAGGTAGAAAAGG TTGGCCTTTTCTACCTGGTCTGG CTTCATTGGCCTTTTCTACCTGG CAGGTAGAAAAGGCCAATGAAGG TTACACCCTATGAGCCAACATGG TACACCCTATGAGCCAACATGGG CTATCCCATGTTGGCTCATAGGG sequence:8011 sequence: 8038 sequence: 8054 sequence:8063 sequence: 8069 sequence:8081 sequence: 8084 sequence: 8102 sequence: 8111 sequence: 8112 sequence: 8119 sequence: 8120 sequence: 8121 sequence: 8122 sequence: 8126 sequence: 8143 sequence: 8146 sequence: 8152 sequence: 8153 sequence: 8164 sequence: 8171 sequence: 8174 sequence: 8359 sequence: 8362 sequence: 8366 sequence:8373 sequence: 8379 sequence: 8389 sequence:8413 sequence: 8414 sequence: 8415 sequence: 8418 sequence: 8419 sequence: 8439 sequence: 8443 sequence: 8449 sequence: 8492 sequence: 8508 sequence: 8511 sequence: 8514 sequence: 8541 sequence: 8548 sequence: 8557 sequence: 8575 sequence: 8577 sequence: 8617 sequence: 8618 sequence: 8660 sequence: 8670 sequence: 8677 sequence:8688 sequence: 8693 sequence:8694 sequence:8706 sequence: 8707 sequence: 8710 sequence: 8711 sequence: 8716 sequence: 8728 sequence: 8734 sequence: 8744 sequence: 8749 sequence: 8778 sequence: 8782 sequence: 8787 sequence:8791 sequence:8796 sequence: 8797 sequence: 8836 sequence:8837 sequence: 8841
3' Tat 3' Rev

3' Rev

3' Rev

3' Rev

3' Rev

3' Rev

3' Rev

3' Rev

3' Rev

3' Rev

3' Rev

3' Rev

3' Rev

3' Rev

3' Rev

3' Rev

3' Rev

3' Rev

3' Rev

3' Rev

3' Rev

3' Rev

Nef

Nef

Nef

Nef

Nef

Nef

Nef

Nef

Nef

Nef

Nef

Nef

Nef

Nef

Nef

Nef

Nef

Nef

Nef

Nef

Nef

Nef

Nef

Nef

Nef

LTR U3 Nef

LTR U3 Nef

LTR U3 Nef

LTR U3 Nef

LTR U3 Nef

LTR U3 Nef

LTR U3 Nef

LTR U3 Nef

LTR U3 Nef

LTR U3 Nef

LTR U3 Nef

LTR U3 Nef

LTR U3 Nef

LTR U3 Nef

LTR U3 Nef

LTR U3 Nef

LTR U3 Nef

LTR U3 Nef

LTR U3 Nef

LTR U3 Nef

LTR U3 Nef

LTR U3 Nef

LTR U3 Nef

LTR U3 Nef 
TCTATCCCATGTTGGCTCATAGG

CCAACATGGGATAGATGACCCGG CGGAGAAAGAAGTATTAGTGTGG CCGCCTAGCATTTCATCACATGG GGGCCATGTGATGAAATGCTAGG CATGGCCCGAGAGATACATCCGG GTACTCCGGATGTATCTCTCGGG AGTACTCCGGATGTATCTCTCGG CAGCAGTTCTTGTAGTACTCCGG ACTACAAGAACTGCTGACACCGG CAGCATTTCTTGTAGTACTCCGG GCTGACACCGAGCTTCTACAAGG CTGACACCGAGCTTCTACAAGGG GAAAGTCCCTTGTAGAAGCTCGG TTCTACAAGGGACTTTCCGCTGG TCTACAAGGGACTTTCCGCTGGG CTACAAGGGACTTTCCGCTGGGG CTTTCCGCTGGGGACTTTCCAGG TTTCCGCTGGGGACTTTCCAGGG CCGCTGGGGACTTTCCAGGGAGG GGGGACTTTCCAGGGAGGTGTGG TTTCCAGGGAGGTGTGGCCTGGG AGGTGTGGCCTGGGCGGAACAGG GGTGTGGCCTGGGCGGAACAGGG GTGTGGCCTGGGCGGAACAGGGG ACCACTCCCCTGTTCCGCCCAGG TGCTTATATGCAGCATCTGAGGG CTGCTTATATGCAGCATCTGAGG CAGCTGCTTTTTGCCTGTACTGG AGCTGCTTTTTGCCTGTACTGGG TAACTGGAGAGACCCAGTACAGG AGGCTCAGATCTGGTCTAACTGG AGTTAGACCAGATCTGAGCCTGG GTTAGACCAGATCTGAGCCTGGG AGAGCTCCCAGGCTCAGATCTGG ATCTGAGCCTGGGAGCTCTCTGG CAGCTAGCCAGAGAGCTCCCAGG CTGGGAGCTCTCTGGCTAGCTGG TGGGAGCTCTCTGGCTAGCTGGG GGGAGCTCTCTGGCTAGCTGGGG TAGATTGAGGCTTAAGCAGTGGG ATAGATTGAGGCTTAAGCAGTGG TTAGGTGACCCTATAGATTGAGG GGTCACCTAAATACACTGTTTGG TTGATCCAGACCAGGTAGAAAGG ATTGGCCTTTCTACCTGGTCTGG CCAGGTAGAAAGGCCAATGAAGG CAGGTAGAAAGGCCAATGAAGGG AACAGTTGTTCTCCCTTCATTGG GTTCACCCTATGAGCCAACATGG TTCACCCTATGAGCCAACATGGG GCCAACATGGGATAGATACCCGG TCCGGGTATCTATCCCATGTTGG sequence: 8842 sequence: 8850 sequence: 8870 sequence: 8904 sequence: 8907 sequence: 8922 sequence: 8927 sequence: 8928 sequence: 8941 sequence: 8948 sequence: 8967 sequence: 8986 sequence: 8987 sequence: 8993 sequence:8999 sequence:9000 sequence:9001 sequence:9011 sequence:9012 sequence:9015 sequence: 9020 sequence:9026 sequence:9035 sequence:9036 sequence:9037 sequence:9043 sequence:9069 sequence: 9070 sequence: 9090 sequence:9091 sequence:9103 sequence:9119 sequence:9121 sequence:9122 sequence:9128 sequence:9132 sequence:9139 sequence:9140 sequence:9141 sequence:9142 sequence:9167 sequence:9168 sequence:9180 sequence:9193 sequence:9239 sequence: 9244 sequence:9249 sequence:9250 sequence:9262 sequence:9286 sequence:9287 sequence:9299 sequence:9300
LTR U3 Nef LTR U3 Nef LTR U3 Nef LTR U3 Nef LTR U3 Nef LTR U3 Nef LTR U3 Nef LTR U3 Nef LTR U3 Nef LTR U3 Nef LTR U3 Nef LTR U3 LTR U3 LTR U3 LTR U3 LTR U3 LTR U3 LTR U3 LTR U3 LTR U3 LTR U3 LTR U3 LTR U3 LTR U3 LTR U3 LTR U3 LTR U3 LTR U3 LTR U3 LTR U3 LTR U3 LTR R LTR R LTR R LTR R LTR R LTR R LTR R LTR R LTR R LTR R LTR R LTR R LTR R LTR U5 LTR U5 LTR U5 LTR U5 LTR U5 LTR U5 LTR U5 LTR U5 LTR U5 


\begin{tabular}{|c|c|c|c|c|}
\hline HIV-1 1058 Target sequence & 5' end of gRNA target site & Strand & \% GC content & HIV-1 target \\
\hline GCGGGGGAAAATTAGATACATGG & sequence:14 & + & 45 & Gag \\
\hline CGGGGGAAAATTAGATACATGGG & sequence:15 & + & 40 & Gag \\
\hline CATGGGAGAAAATTCGGTTAAGG & sequence:32 & + & 40 & Gag \\
\hline GAGAAAATTCGGTTAAGGCCAGG & sequence:37 & + & 45 & Gag \\
\hline AGAAAATTCGGTTAAGGCCAGGG & sequence:38 & + & 40 & Gag \\
\hline GAAAATTCGGTTAAGGCCAGGGG & sequence:39 & + & 45 & Gag \\
\hline AAAATTCGGTTAAGGCCAGGGGG & sequence:40 & + & 45 & Gag \\
\hline AACATATAGTATGGGCAAGCAGG & sequence:83 & + & 40 & Gag \\
\hline ACATATAGTATGGGCAAGCAGGG & sequence: 84 & + & 40 & Gag \\
\hline GAACGATTTGCACTTAACCCTGG & sequence:112 & + & 45 & Gag \\
\hline CTGCTGTTTCTAACAGGCCAGGG & sequence:129 & - & 50 & Gag \\
\hline TCTGCTGTTTCTAACAGGCCAGG & sequence:130 & - & 50 & Gag \\
\hline AGCCTTCTGCTGTTTCTAACAGG & sequence:135 & - & 45 & Gag \\
\hline CTACAGCCATCCCTTCAAACAGG & sequence:178 & + & 50 & Gag \\
\hline TCTGATCCTGTTTGAAGGGATGG & sequence:184 & - & 45 & Gag \\
\hline GTTCTTCTGATCCTGTTTGAAGG & sequence:189 & - & 40 & Gag \\
\hline СССTCTATTGTGTACATCAAAGG & sequence:239 & + & 40 & Gag \\
\hline AGAGGTAAGAGACACCAAAGAGG & sequence:264 & + & 45 & Gag \\
\hline AGAGGCTTTAGACAAGATAGAGG & sequence:282 & + & 40 & Gag \\
\hline TGACACAGGAAACAGCAGCCAGG & sequence:354 & + & 55 & Gag \\
\hline TAGGGTAATTTTGGCTGACCTGG & sequence:372 & - & 45 & Gag \\
\hline CTTGGAGGTTCTGCACTATAGGG & sequence:390 & - & 45 & Gag \\
\hline CCTATAGTGCAGAACCTCCAAGG & sequence:391 & + & 50 & Gag \\
\hline GCAGAACCTCCAAGGGCAAATGG & sequence:399 & + & 55 & Gag \\
\hline GATGTACCATTTGCCCTTGGAGG & sequence:405 & - & 50 & Gag \\
\hline CCAAGGGCAAATGGTACATCAGG & sequence:408 & + & 50 & Gag \\
\hline GCAAACATGGGTATTACTTCTGG & sequence: 490 & - & 40 & Gag \\
\hline GGTGTTTAAATCTTGTGGGGTGG & sequence:530 & - & 45 & Gag \\
\hline AAACACCATGCTAAACACAGTGG & sequence:546 & + & 40 & Gag \\
\hline AACACCATGCTAAACACAGTGGG & sequence:547 & + & 40 & Gag \\
\hline ACACCATGCTAAACACAGTGGGG & sequence:548 & + & 45 & Gag \\
\hline CACCATGCTAAACACAGTGGGGG & sequence:549 & + & 50 & Gag \\
\hline ACCATGCTAAACACAGTGGGGGG & sequence:550 & + & 50 & Gag \\
\hline TCCCCCCACTGTGTTTAGCATGG & sequence:551 & - & 55 & Gag \\
\hline TTCTGCAGCTTCCTCATTGATGG & sequence:605 & - & 45 & Gag \\
\hline TCAATGAGGAAGCTGCAGAATGG & sequence:608 & + & 45 & Gag \\
\hline CAATGAGGAAGCTGCAGAATGGG & sequence:609 & + & 45 & Gag \\
\hline AGAATACATCCAGCGCAAGCAGG & sequence:634 & + & 50 & Gag \\
\hline GAATACATCCAGCGCAAGCAGGG & sequence:635 & + & 50 & Gag \\
\hline GCTATAGGCCCTGCTTGCGCTGG & sequence:643 & - & 65 & Gag \\
\hline CAAGCAGGGCCTATAGCACCAGG & sequence:649 & + & 60 & Gag \\
\hline CTTGGGTCTCTTATCTGGCCTGG & sequence:667 & - & 55 & Gag \\
\hline CAGGCCAGATAAGAGACCCAAGG & sequence:668 & + & 55 & Gag \\
\hline AGGCCAGATAAGAGACCCAAGGG & sequence:669 & + & 50 & Gag \\
\hline GGCCAGATAAGAGACCCAAGGGG & sequence:670 & + & 55 & Gag \\
\hline TTCCCCTTGGGTCTCTTATCTGG & sequence:672 & - & 50 & Gag \\
\hline CTGCTATGTCACTTCCCCTTGGG & sequence:684 & - & 50 & Gag \\
\hline CTTATCTGGCCTGGTGCTATAGG & sequence:658 & - & 50 & Gag \\
\hline CCAAGGGGAAGTGACATAGCAGG & sequence:685 & + & 55 & Gag \\
\hline AGGAACTACTAGTACCCTTCAGG & sequence:705 & + & 45 & Gag \\
\hline CCCTTCAGGAACAAATAACATGG & sequence:719 & + & 40 & Gag \\
\hline AATAATCCACCTATCCCAGTAGG & sequence:748 & + & 40 & Gag \\
\hline АTTTCTCCTACTGGGATAGGTGG & sequence:754 & - & 45 & Gag \\
\hline TAGATTTCTCCTACTGGGATAGG & sequence:757 & - & 40 & Gag \\
\hline GTATAGTCCTACCAGCATTCTGG & sequence:822 & + & 45 & Gag \\
\hline CTTATGTCCAGAATGCTGGTAGG & sequence:829 & - & 45 & Gag \\
\hline AGCATTCTGGACATAAGACAAGG & sequence:835 & + & 40 & Gag \\
\hline GGACATAAGACAAGGACCAAAGG & sequence:843 & + & 45 & Gag \\
\hline AAGGACCAAAGGAACCCTTTAGG & sequence:854 & + & 45 & Gag \\
\hline AGGACCAAAGGAACCCTTTAGGG & sequence:855 & + & 45 & Gag \\
\hline TAGTCCCTAAAGGGTTCCTTTGG & sequence:859 & - & 45 & Gag \\
\hline CGGTCTACATAGTCCCTAAAGGG & sequence:868 & - & 45 & Gag \\
\hline CCTTTAGGGACTATGTAGACCGG & sequence:869 & + & 45 & Gag \\
\hline AAGAGCCGAGCAAGCTTCACAGG & sequence:906 & + & 55 & Gag \\
\hline AGCCGAGCAAGCTTCACAGGAGG & sequence:909 & + & 60 & Gag \\
\hline TACCTCCTGTGAAGCTTGCTCGG & sequence:911 & - & 50 & Gag \\
\hline TTGGATGACAGAAACCTTGTTGG & sequence:939 & + & 40 & Gag \\
\hline GTTCGCGTTTTGGACCAACAAGG & sequence:953 & - & 50 & Gag \\
\hline TACAATCTGGGTTCGCGTTTTGG & sequence:963 & - & 45 & Gag \\
\hline AGGACCAGCAGCTACACTAGAGG & sequence:1005 & + & 55 & Gag \\
\hline
\end{tabular}


ATTTCCTCTAGTGTAGCTGCTGG GGAAATGATGACAGCATGTCAGG GAAATGATGACAGCATGTCAGGG GATGACAGCATGTCAGGGAGTGG ATGACAGCATGTCAGGGAGTGGG ACAGCATGTCAGGGAGTGGGAGG TGTCAGGGAGTGGGAGGACCTGG AAAACTCTTGCTTTATGGCCAGG TGGCCATAAAGCAAGAGTTTTGG CAGCCAAAACTCTTGCTTTATGG TGGCACCTGAATTTGTTACTTGG GGTGCCATAATGATGCAGAAAGG GAAAGGCAATTTTAGGAACCAGG GGCACATAGCCAAAAATTGCAGG GCACATAGCCAAAAATTGCAGGG CCAAAAATTGCAGGGCCCCTAGG TTGCAGGGCCCCTAGGAAGAAGG TGCAGGGCCCCTAGGAAGAAGGG TCCAACAGCCCTTCTTCCTAGGG TTCCAACAGCCCTTCTTCCTAGG AAGAAGGGCTGTTGGAAATGTGG GAAAGATTGTGATCAGAGACAGG AAGATCTGGCCTTCCCACAAAGG TCTGGCCTTCCCACAAAGGAAGG CCTTCCCACAAAGGAAGGCCAGG AATTCCCTGGCCTTCCTTTGTGG CTGCTCTGAAGAAAATTCCCTGG GCTGGTGGGGCTGTTGGCTCTGG CTCTCTGCTGGTGGGGCTGTTGG CCCCACCAGCAGAGAGCTTCAGG CCAGCAGAGAGCTTCAGGTTTGG CAGCAGAGAGCTTCAGGTTTGGG AGCAGAGAGCTTCAGGTTTGGGG AGAGAGCTTCAGGTTTGGGGAGG AACAACTCCCTCTCAGAAGCAGG TGTGGCTCCTGCTTCTGAGAGGG TTGTGGCTCCTGCTTCTGAGAGG AGTGATCTGAGGGAAGCTAAAGG GCTTCCCTCAGATCACTCTTTGG GTTGCCAAAGAGTGATCTGAGGG CGTTGCCAAAGAGTGATCTGAGG CCCCTCGTCACAATAAAGATAGG CCCTCGTCACAATAAAGATAGGG CCTCGTCACAATAAAGATAGGGG CTCGTCACAATAAAGATAGGGGG TCGTCACAATAAAGATAGGGGGG AAAGATAGGGGGGCAGCTAAAGG AAATGAATTTGCCCGGAAGATGG CCAAAAATGATAGGGGGAATTGG AAAATGATAGGGGGAATTGGAGG CCTACACCTGTCAACATAATTGG GTACCAGTAAAATTGAAGCCAGG AAATTGAAGCCAGGAATGGATGG ACTTTTGGACCATCCATTCCTGG TGTGCAGAAATGGAAAAGGAAGG GTGCAGAAATGGAAAAGGAAGGG GACTTCTGGGAAGTTCAATTAGG TTAGGAATACCACATCCCGCAGG TAGGAATACCACATCCCGCAGGG TTTTTTAACCCTGCGGGATGTGG ATCAGTAACAGTACTGGATGTGG TCAGTAACAGTACTGGATGTGGG CTCGTTGTTTACACTAGGTATGG GGTGTCTCGTTGTTTACACTAGG AGTGTAAACAACGAGACACCAGG GTGTAAACAACGAGACACCAGGG TCAGTATAATGTGCTTCCACAGG CAGTATAATGTGCTTCCACAGGG ATAATGTGCTTCCACAGGGATGG GTGCTTCCACAGGGATGGAAAGG GGTGATCCTTTCCATCCCTGTGG sequence:1009 sequence: 1026 sequence: 1027 sequence:1032 sequence: 1033 sequence:1036 sequence: 1042 sequence: 1060 sequence:1062 sequence:1065 sequence:1098 sequence:1114 sequence: 1131 sequence:1184 sequence: 1185 sequence: 1193 sequence: 1200 sequence:1201 sequence:1209 sequence: 1210 sequence:1216 sequence: 1257 sequence:1294 sequence:1298 sequence: 1303 sequence:1308 sequence: 1321 sequence: 1345 sequence:1351 sequence: 1358 sequence: 1363 sequence:1364 sequence: 1365 sequence: 1368 sequence:1395 sequence: 1402 sequence:1403 sequence: 1432 sequence:1438 sequence:1442 sequence: 1443 sequence:1467 sequence:1468 sequence:1469 sequence:1470 sequence: 1471 sequence:1481 sequence:1546 sequence: 1572 sequence: 1575 sequence:1677 sequence:1761 sequence: 1770 sequence: 1779 sequence: 1851 sequence:1852 sequence:1995 sequence: 2013 sequence:2014 sequence: 2022 sequence:2051 sequence: 2052 sequence:2131 sequence:2136 sequence: 2139 sequence: 2140 sequence:2171 sequence: 2172 sequence: 2176 sequence: 2181 sequence: 2187 
TGAGACAACATCTGTTGAAGTGG GAGACAACATCTGTTGAAGTGGG AGACAACATCTGTTGAAGTGGGG AGAAAGAACCTCCATTCCTTTGG AGAACCTCCATTCCTTTGGATGG GAACCTCCATTCCTTTGGATGGG AACCTCCATTCCTTTGGATGGGG TACCCCATCCAAAGGAATGGAGG TCATACCCCATCCAAAGGAATGG GGAGTTCATACCCCATCCAAAGG GTACTGTCCATTTATCAGGATGG GGCTGTACTGTCCATTTATCAGG TCTTTCTCTGGCAGCACTATAGG TGCTGCCAGAGAAAGACACCTGG ACAGTCCAGGTGTCTTTCTCTGG TGGGCAAGTCAGATTTATGCAGG GGGCAAGTCAGATTTATGCAGGG GGCACTTATGTAAACTCCTTAGG TTAGTGCTTTGGTTCCCCTAAGG TAGCAGAAATACAGAAGCAGGGG GAAATACAGAAGCAGGGGCAAGG AGAAGCAGGGGCAAGGCCAATGG AGGAAACATGGGAATCATGGTGG CAGATTATTGGCAAGCCACCTGG AAGCCACCTGGATTCCTGAGTGG AGCCACCTGGATTCCTGAGTGGG AAATTCCCACTCAGGAATCCAGG GTATTGACAAATTCCCACTCAGG CCCCTCCCTTAGTGAAATTATGG CTATGGGTTCTTTCTCTAACTGG AAGGTTTCTGCTCCTACTATGGG GAAGGTTTCTGCTCCTACTATGG GCAGAAACCTTCTACGTAGATGG CAGAAACCTTCTACGTAGATGGG AGAAACCTTCTACGTAGATGGGG AGCTGCCCCATCTACGTAGAAGG ACGTAGATGGGGCAGCTAACAGG CGTAGATGGGGCAGCTAACAGGG GCTAACAGGGAGACTAAACTAGG GAGACTAAACTAGGAAAAGCAGG GCAGGATATGTTACCGACAGAGG TACCGACAGAGGAAGACAAAAGG AACCTTTTGTCTTCCTCTGTCGG CTGATTTGTTGTGTCAGTCAGGG AGCAATTCATCTAGCACTGCAGG CATCTAGCACTGCAGGATTCAGG AGGAAAAGGTCTACCTGACATGG TGTGTGCTGGTACCCATGTCAGG ACATGGGTACCAGCACACAAAGG CCAGCACACAAAGGAATTGGGGG AATTAGTCAGTGCTGGGATCAGG AGATGGAATAGATAAGGCCCAGG CTTTTGCTACTATAGGTGGCAGG TAGCTGACATTTATCACAGCTGG CTAAAAGGGGAAGCCATGCATGG GGACAAGTAGACTGTAGTCCAGG TAGACTGTAGTCCAGGAATATGG CAATCTAGTTGCCATATTCCTGG TGGCTACATGAACTGCTACCAGG GTAGCAGTTCATGTAGCCAGTGG TAGCAGTTCATGTAGCCAGTGGG TTCTGCTTCTATATACCCACTGG GAAGTGATCCCAGCAGAGACAGG AAGTGATCCCAGCAGAGACAGGG TTTCCTGCCCTGTCTCTGCTGGG GTTTCCTGCCCTGTCTCTGCTGG CAGCAACTTCACCAGTAGTGCGG CTTCACCAGTAGTGCGGTCAAGG GGCGGCCTTGACCGCACTACTGG GTGCGGTCAAGGCCGCCTGTTGG CGGTCAAGGCCGCCTGTTGGTGG sequence:2353

sequence: 2354

sequence: 2355

sequence: 2404

sequence: 2408

sequence: 2409

sequence: 2410

sequence: 2412

sequence: 2415

sequence: 2420

sequence: 2441

sequence: 2445

sequence: 2466

sequence: 2473

sequence: 2478

sequence: 2535

sequence: 2536

sequence: 2569

sequence: 2585

sequence: 2716

sequence: 2721

sequence: 2728

sequence: 2923

sequence: 2947

sequence: 2959

sequence: 2960

sequence: 2965

sequence: 2973

sequence: 2995

sequence: 3020

sequence:3036

sequence:3037

sequence:3048

sequence:3049

sequence: 3050

sequence:3055

sequence:3061

sequence:3062

sequence:3075

sequence: 3084

sequence:3102

sequence:3113

sequence: 3115

sequence:3142

sequence:3179

sequence: 3186

sequence: 3322

sequence:3335

sequence:3339

sequence: 3348

sequence: 3388

sequence: 3425

sequence: 3500

sequence: 3532

sequence:3552

sequence: 3573

sequence: 3580

sequence:3591

sequence: 3638

sequence: 3642

sequence:3643

sequence: 3658

sequence: 3678

sequence:3679

sequence: 3686

sequence: 3687

sequence: 3773

sequence: 3779

sequence: 3784

sequence: 3790

sequence: 3793
Pol

Pol

Pol

Pol

Pol

Pol

Pol

Pol

Pol

Pol

Pol

Pol

Pol

Pol

Pol

Pol

Pol

Pol

Pol

Pol

Pol

Pol

Pol

Pol

Pol

Pol

Pol

Pol

Pol

Pol

Pol

Pol

Pol

Pol

Pol

Pol

Pol

Pol

Pol

Pol

Pol

Pol

Pol

Pol

Pol

Pol

Pol

Pol

Pol

Pol

Pol

Pol

Pol

Pol

Pol

Pol

Pol

Pol

Pol

Pol

Pol

Pol

Pol

Pol

Pol

Pol

Pol

Pol

Pol

Pol

Pol 
GGTCAAGGCCGCCTGTTGGTGGG CAAGGCCGCCTGTTGGTGGGCGG AAGGCCGCCTGTTGGTGGGCGGG CTTGACCCCCGCCCACCAACAGG TTGGTGGGCGGGGGTCAAGCAGG GCGGGGGTCAAGCAGGAATTTGG CCCTACAATCCCCAAAGTCAAGG TCCTTGACTTTGGGGATTGTAGG TCTACTACTCCTTGACTTTGGGG TATAGGACAGGTAAGAGATCAGG TTTAAAAGAAGAGGGGGGATTGG TTAAAAGAAGAGGGGGGATTGGG TAAAAGAAGAGGGGGGATTGGGG GGGATTGGGGACTATAGTGCAGG GGATTGGGGACTATAGTGCAGGG GATTGGGGACTATAGTGCAGGGG GGGACAGCAGAGATCCACTTTGG AGCAGAGATCCACTTTGGAAAGG TTTGCTGGTCCTTTCCAAAGTGG AAGGACCAGCAAAGCTCCTCTGG CCAGCAAAGCTCCTCTGGAAAGG AAGCTCCTCTGGAAAGGTGAAGG AGCTCCTCTGGAAAGGTGAAGGG GCTCCTCTGGAAAGGTGAAGGGG CTGCCCCTTCACCTTTCCAGAGG GATTATGGAAAACAGATGGCAGG GATGGCAGGTGATGATTGTATGG TGATTGTATGGCAAGTAGACAGG TATGGCAAGTAGACAGGATGAGG ACAGGATGAGGATTAGAACATGG TATCAAGGAAAGCTAAGGACTGG AGAAGTACACATCCCACTAGAGG CCCACTAGAGGATGCTGAATTGG ACCAATTCAGCATCCTCTAGTGG ACATATTGGGGTCTGCATACAGG TGCATACAGGAGAAAGAGAATGG AGGAGAAAGAGAATGGCATTTGG GTCAGGGAGTCTCCATAGAATGG AGGGAGTCTCCATAGAATGGAGG TTAGTTGGTCTGCTAGGTCAGGG ATTAGTTGGTCTGCTAGGTCAGG GATGAATTAGTTGGTCTGCTAGG GCCATAAGAAATGCCCTGTTAGG TCCTAACAGGGCATTTCTTATGG CATAGGTGTGAATATCAAGCAGG CAAGCAGGACATAACAAGGTAGG GGTAGGATCTTTACAGTACTTGG AAAGGTGGCTTTCTCCTTTTTGG TTCGCAACACTAGGCAAAGGTGG AGTTTCGCAACACTAGGCAAAGG GCCTAGTGTTGCGAAACTGACGG TCCGTCAGTTTCGCAACACTAGG TAGTGTTGCGAAACTGACGGAGG CGAAACTGACGGAGGACAGATGG GAACAAGTCCCAGCAGACCAAGG AACAAGTCCCAGCAGACCAAGGG TCTGTGGCCCTTGGTCTGCTGGG AGCAGACCAAGGGCCACAGAGGG ATGGCTCCCTCTGTGGCCCTTGG TCATTGTATGGCTCCCTCTGTGG AGAGGGAGCCATACAATGAATGG TGAGACACTTTCCCAGGATATGG AAGTTGTGAAGCCATATCCTGGG TAAGTTGTGAAGCCATATCCTGG AGGATATGGCTTCACAACTTAGG ACTTATGGGGATACTTGGGTAGG TGGGGATACTTGGGTAGGAGTGG GGATGTCAACATAGCAGAATAGG GAATAGGCATTATTCGACAGAGG CGACAGAGGAGAGCAAGAAATGG GGCTCTAGTTTAGGGTCTACTGG sequence: 3794 sequence: 3797 sequence: 3798 sequence:3805 sequence: 3809 sequence: 3816 sequence: 3843 sequence: 3844 sequence: 3852 sequence: 3902 sequence: 3972 sequence: 3973 sequence: 3974 sequence: 3987 sequence: 3988 sequence:3989 sequence: 4102 sequence: 4107 sequence: 4116 sequence: 4126 sequence: 4131 sequence:4137 sequence: 4138 sequence:4139 sequence: 4142 sequence: 4227 sequence: 4241 sequence: 4253 sequence:4259 sequence: 4271 sequence: 4322 sequence:4389 sequence: 4401 sequence:4402 sequence: 4432 sequence: 4445 sequence: 4452 sequence: 4475 sequence: 4478 sequence: 4527 sequence: 4528 sequence: 4533 sequence: 4585 sequence: 4586 sequence: 4621 sequence:4636 sequence: 4653 sequence: 4696 sequence: 4711 sequence: 4714 sequence:4719 sequence: 4720 sequence: 4722 sequence: 4730 sequence: 4752 sequence: 4753 sequence: 4760 sequence: 4763 sequence:4769 sequence: 4776 sequence: 4780 sequence: 4840 sequence: 4851 sequence: 4852 sequence: 4854 sequence: 4893 sequence: 4898 sequence: 4971 sequence: 4987 sequence:5001 sequence:5026
Pol

Pol Pol Pol Pol Pol Pol Pol Pol Pol

Pol cPPT

Pol cPPT

Pol cPPT

Pol cPPT

Pol cPPT

Pol cPPT

Pol

Pol

Pol

Pol

Pol

Pol

Pol

Pol

Pol

Pol

Pol Vif

Pol Vif

Pol Vif

Pol Vif

Vif

Vif

Vif

Vif

Vif

Vif

Vif

Vif

Vif

Vif

Vif

Vif

Vif

Vif

Vif

Vif

Vif

Vif

Vif

Vif

Vif

Vif

Vif

Vif

Vif $\mathrm{Vpr}$

Vif Vpr

Vif Vpr

Vif Vpr

Vif Vpr

Vif $V p r$

Vif $\mathrm{Vpr}$

$\mathrm{Vpr}$

Vpr

Vpr

Vpr

Vpr

Vpr

Vpr

Vpr

Vpr

Vpr 
TAGACCCTAAACTAGAGCCCTGG GCTTCCAGGGCTCTAGTTTAGGG TGCTTCCAGGGCTCTAGTTTAGG CTAGAGCCCTGGAAGCATCCAGG TGACTTCCTGGATGCTTCCAGGG CTGACTTCCTGGATGCTTCCAGG AGCATCCAGGAAGTCAGCCTAGG GCAGTCCTAGGCTGACTTCCTGG CAATTGGTACAAGCAGTCCTAGG AAAGGCTTAGGCATCTCCTATGG GCTTAGGCATCTCCTATGGCAGG TCTCCTATGGCAGGAAGAAGCGG TCTCCGCTTCTTCCTGCCATAGG TGATCAATCTGACTGTCTGGAGG GCTTGATCAATCTGACTGTCTGG ACAGGTACTACTCACTGCTTTGG GATAGTGGCAATGAGAGTGAAGG ATAGTGGCAATGAGAGTGAAGGG TAGTGGCAATGAGAGTGAAGGGG CAATGAGAGTGAAGGGGATCAGG TGAGAGTGAAGGGGATCAGGAGG GGAGGAATTGTCAGCACTCGTGG ATTGTCAGCACTCGTGGAGATGG TTGTCAGCACTCGTGGAGATGGG TGTCAGCACTCGTGGAGATGGGG ACTACGCTCACCATGCTCCTTGG CATCAATATTCCAAGGAGCATGG GTAGAGCTGCAGAACAGTTGTGG GGGAGCAGCAGGAAGCACTATGG GGAGCAGCAGGAAGCACTATGGG CGCAGCGTCAATGACGCTGACGG GTCAATGACGCTGACGGTACAGG CAGGCCAGACTATTATTGTCTGG AACAGCAGAACAACTTGCTGAGG ACAGCAGAACAACTTGCTGAGGG CAACTTGCTGAGGGCTATTGAGG ACAACATCTGTTGCAACTCACGG ATCTGTTGCAACTCACGGTCTGG TCTGTTGCAACTCACGGTCTGGG CTGTTGCAACTCACGGTCTGGGG CTGGGGCATCAAGCAGCTCCAGG GCAGCTCCAGGCAAGAGTCCTGG CCAGGCAAGAGTCCTGGCTGTGG CTAGGTATCTTTCCACAGCCAGG GGCTGTGGAAAGATACCTAGAGG ACCCGCCTCCCAACCCAGAGGGG TCCCCTCTGGGTTGGGAGGCGGG GTCCCCTCTGGGTTGGGAGGCGG TGTCGGGTCCCCTCTGGGTTGGG CTGTCGGGTCCCCTCTGGGTTGG CAACCCAGAGGGGACCCGACAGG TCTGTTCCTTCGGGCCTGTCGGG TTCTGTTCCTTCGGGCCTGTCGG CCCGAAGGAACAGAAGAAGAAGG ACCTTCTTCTTCTGTTCCTTCGG GAGAGAGACAGAGACAGATCCGG AGACAGATCCGGACCATTAGTGG AGATCCGGACCATTAGTGGATGG GAATCCATCCACTAATGGTCCGG GCTAAGAATCCATCCACTAATGG CAATCATCTGGGTCGATCTACGG AGTCTCTCAATCGGTGGTAGAGG AGAGTAAGTCTCTCAATCGGTGG ACTTACTCTTGATTGTGACGAGG CTTGATTGTGACGAGGACTCTGG GACGAGGACTCTGGAACTTCTGG ACGAGGACTCTGGAACTTCTGGG CTCTGGAACTTCTGGGACGCAGG TCTGGAACTTCTGGGACGCAGGG CTGGAACTTCTGGGACGCAGGGG TGGAACTTCTGGGACGCAGGGGG sequence: 5030

sequence:5034

sequence:5035

sequence: 5041

sequence: 5047

sequence: 5048

sequence:5054

sequence:5059

sequence:5071

sequence: 5140

sequence:5144

sequence: 5153

sequence:5156

sequence:5194

sequence:5197

sequence:5227

sequence: 5404

sequence:5405

sequence:5406

sequence: 5412

sequence: 5415

sequence: 5433

sequence:5439

sequence: 5440

sequence:5441

sequence:5465

sequence: 5475

sequence: 5502

sequence: 6982

sequence: 6983

sequence:7006

sequence:7012

sequence:7031

sequence:7062

sequence: 7063

sequence:7072

sequence:7099

sequence:7104

sequence:7105

sequence:7106

sequence: 7123

sequence:7135

sequence: 7141

sequence: 7153

sequence:7156

sequence: 7568

sequence:7569

sequence: 7570

sequence: 7576

sequence: 7577

sequence:7578

sequence: 7592

sequence:7593

sequence:7601

sequence:7602

sequence:7628

sequence:7639

sequence:7643

sequence: 7647

sequence: 7652

sequence: 7674

sequence: 7711

sequence:7717

sequence:7731

sequence: 7738

sequence: 7747

sequence:7748

sequence: 7755

sequence: 7756

sequence: 7757

sequence: 7758
Vpr 5' Tat Vpr 5' Tat Vpr 5' Tat 5' Tat 5' Tat 5 ' Tat 5' Tat 5' Tat 5 ' Tat 5' Tat 5' Tat 5' Tat 5' Tat 5' Tat 5' Rev 5' Tat 5' Rev 5' Tat 5' Rev Vpu Vpu Vpu Vpu Vpu Vpu Vpu Vpu Vpu Vpu Vpu Vpu RRE RRE RRE RRE RRE RRE RRE RRE RRE RRE RRE RRE RRE RRE RRE RRE RRE

3' Tat 3' Rev 3' Tat 3' Rev 3' Tat 3' Rev 3' Tat 3' Rev 3' Tat 3' Rev 3' Tat 3' Rev 3' Tat 3' Rev 3' Tat 3' Rev 3' Tat 3' Rev 3' Tat 3' Rev 3' Tat 3' Rev 3' Tat 3' Rev 3' Tat $3^{\prime}$ Rev 3' Tat 3' Rev 3' Tat 3' Rev 3' Rev 3' Rev 3' Rev 3' Rev 3' Rev 3' Rev 3' Rev 3' Rev 3' Rev 3' Rev 3' Rev 
ACTTCTGGGACGCAGGGGGTGGG GGTGGGAGATCCTCAAATATTGG GGGAGATCCTCAAATATTGGTGG GGAGATTCCACCAATATTTGAGG TCTCCTACAGTATTGGAGTCAGG GTTCCTGACTCCAATACTGTAGG GTGGTCAAAACGTAGTATTGTGG CAAAACGTAGTATTGTGGGATGG TGCTGGCTCAGTTGCTGGTGCGG TCTGCTGCTGGCTCAGTTGCTGG GCAACTGAGCCAGCAGCAGAAGG CAACTGAGCCAGCAGCAGAAGGG TGAGCCAGCAGCAGAAGGGGTGG GAGCCAGCAGCAGAAGGGGTGGG GCTCCCACCCCTTCTGCTGCTGG GGGAGCAGCATCTCGAGACCTGG TCTCGAGACCTGGAAAGACATGG TGAGTGCTCCATGTCTTTCCAGG CTAACAATGCTGCTTGTGCCTGG TGCCTGGCTAGAAGCACAAGAGG ATCCTCTTGTGCTTCTAGCCAGG GCTAGAAGCACAAGAGGATGAGG AGAAGCACAAGAGGATGAGGAGG AGCACAAGAGGATGAGGAGGTGG GCACAAGAGGATGAGGAGGTGGG GGGTTTTCCAGTCAGACCTCAGG AAAGGCACCTGAGGTCTGACTGG ATTGGTCTTAAAGGCACCTGAGG AAAGAAAAGGGGGGACTGGAAGG AAGAAAAGGGGGGACTGGAAGGG AATTTACTCCCAGCAAAGACAGG AAGGATATCCTGTCTTTGCTGGG CAAGGATATCCTGTCTTTGCTGG GACAGGATATCCTTGATCTGTGG ACAGGATATCCTTGATCTGTGGG TGTGGTAGACCCACAGATCAAGG CTGTGGGTCTACCACACACAAGG CAGGGAAGTAGCCTTGTGTGTGG CACAAGGCTACTTCCCTGATTGG GTGTGTAATTCTGCCAATCAGGG GGTGTGTAATTCTGCCAATCAGG GATTGGCAGAATTACACACCAGG ATTGGCAGAATTACACACCAGGG CAGAATTACACACCAGGGCCAGG AGAATTACACACCAGGGCCAGGG GGATATCTGGTCCCTGGCCCTGG GTCAGGGGATATCTGGTCCCTGG ACCAGATATCCCCTGACCTTTGG TCCAAAGGTCAGGGGATATCTGG GATATCCCCTGACCTTTGGATGG AAGCACCATCCAAAGGTCAGGGG AAAGCACCATCCAAAGGTCAGGG TAAAGCACCATCCAAAGGTCAGG TAGCTTAAAGCACCATCCAAAGG TCTATCTTCTCTGGTTCAACTGG GTGGCCTCTTCTATCTTCTCTGG AAGATAGAAGAGGCCACTGAAGG GCAGTTGTTTTCTCCTTCAGTGG TTACACCCGATAAACCAGCATGG TACACCCGATAAACCAGCATGGG CCCGATAAACCAGCATGGGATGG TCCATCCCATGCTGGTTTATCGG CCAGCATGGGATGGACGACCCGG ACACTAACACTTCTCTCTCCGGG CACACTAACACTTCTCTCTCCGG CGGAGAGAGAAGTGTTAGTGTGG GTCGCTAGCACTCCGTCACGTGG GCATCTCTCGGGCCACGTGACGG CGTGGCCCGAGAGATGCATCCGG GTACTCCGGATGCATCTCTCGGG AGTACTCCGGATGCATCTCTCGG sequence: 7762 sequence:7779 sequence: 7782 sequence:7789 sequence: 7807 sequence: 7810 sequence:7997 sequence: 8002 sequence: 8053 sequence:8058 sequence: 8061 sequence: 8062 sequence:8066 sequence: 8067 sequence: 8070 sequence:8087 sequence:8097 sequence:8105 sequence:8146 sequence:8162 sequence: 8164 sequence: 8168 sequence: 8171 sequence: 8174 sequence: 8175 sequence: 8195 sequence: 8202 sequence:8211 sequence:8271 sequence: 8272 sequence: 8297 sequence:8305 sequence: 8306 sequence: 8314 sequence: 8315 sequence:8324 sequence: 8331 sequence: 8342 sequence: 8347 sequence: 8360 sequence:8361 sequence:8364 sequence: 8365 sequence: 8370 sequence:8371 sequence:8382 sequence: 8388 sequence: 8394 sequence: 8395 sequence: 8398 sequence:8403 sequence: 8404 sequence:8405 sequence: 8410 sequence: 8436 sequence: 8445 sequence: 8451 sequence: 8464 sequence: 8490 sequence: 8491 sequence: 8495 sequence:8496 sequence: 8504 sequence: 8522 sequence: 8523 sequence: 8524 sequence: 8557 sequence: 8569 sequence: 8575 sequence: 8580 sequence:8581

\section{3' Rev \\ 3' Rev \\ 3' Rev \\ 3' Rev \\ 3' Rev \\ 3' Rev \\ Nef \\ Nef \\ Nef \\ Nef \\ Nef \\ Nef \\ Nef \\ Nef \\ Nef \\ Nef \\ Nef \\ Nef \\ Nef \\ Nef \\ Nef \\ Nef \\ Nef \\ Nef \\ Nef \\ Nef \\ Nef \\ Nef \\ Nef \\ Nef}

LTR U3 Nef

LTR U3 Nef

LTR U3 Nef

LTR U3 Nef

LTR U3 Nef

LTR U3 Nef

LTR U3 Nef

LTR U3 Nef

LTR U3 Nef

LTR U3 Nef

LTR U3 Nef

LTR U3 Nef

LTR U3 Nef

LTR U3 Nef

LTR U3 Nef

LTR U3 Nef

LTR U3 Nef

LTR U3 Nef

LTR U3 Nef

LTR U3 Nef

LTR U3 Nef

LTR U3 Nef

LTR U3 Nef

LTR U3 Nef

LTR U3 Nef

LTR U3 Nef

LTR U3 Nef

LTR U3 Nef

LTR U3 Nef

LTR U3 Nef

LTR U3 Nef

LTR U3 Nef

LTR U3 Nef

LTR U3 Nef

LTR U3 Nef

LTR U3 Nef

LTR U3 Nef

LTR U3 Nef

LTR U3 Nef

LTR U3 Nef

LTR U3 Nef 


\begin{abstract}
GATGCATCCGGAGTACTACAAGG CAGCAGTCCTTGTAGTACTCCGG CTGACATCGAGATTTCTACAAGG TTCTACAAGGGACTTTCCACTGG TCTACAAGGGACTTTCCACTGGG CTACAAGGGACTTTCCACTGGGG ACTTTCCACTGGGGACTTTCCGG CTTTCCACTGGGGACTTTCCGGG TTTCCACTGGGGACTTTCCGGGG CCACTGGGGACTTTCCGGGGAGG TTTCCGGGGAGGCGTGGCCTGGG TGCTTATATGCAGCATCTGAGGG CTGCTTATATGCAGCATCTGAGG CAGCTGCTTTTTGCCTGTACTGG AGCTGCTTTTTGCCTGTACTGGG TCTACAGAGAGACCCAGTACAGG CTGTAGACCAGATCTGAGCCTGG TGTAGACCAGATCTGAGCCTGGG AGAGCTCCCAGGCTCAGATCTGG ATCTGAGCCTGGGAGCTCTCTGG TAGTTAGCCAGAGAGCTCCCAGG TGGGAGCTCTCTGGCTAACTAGG GGGAGCTCTCTGGCTAACTAGGG CTTTATTGAGGCTTAAGCAGTGG ACTCAAGGCAAGCTTTATTGAGG
\end{abstract}

sequence: 8587 sequence: 8594 sequence: 8614 sequence: 8627 sequence: 8628 sequence: 8629 sequence: 8638 sequence: 8639 sequence: 8640 sequence: 8643 sequence: 8654 sequence: 8697 sequence:8698 sequence:8718 sequence: 8719 sequence:8731 sequence: 8747 sequence: 8748 sequence: 8754 sequence:8758 sequence: 8765 sequence: 8767 sequence: 8768 sequence:8794 sequence: 8806
LTR U3 Nef LTR U3 Nef LTR U3 Nef LTR U3 LTR U3 LTR U3 LTR U3 LTR U3 LTR U3 LTR U3 LTR U3 LTR U3 LTR U3 LTR U3 LTR U3 LTR U3 LTR R LTR R LTR R LTR R LTR R LTR R LTR R LTR R LTR R 